\title{
Spectrophotometers for Plutonium Monitoring in HB-Line
}

\author{
R.J. Lascola \\ P.E. O'Rourke \\ E.A. Kyser \\ D.M. Immel \\ J.R. Plummer \\ E.V. Evans
}

February 12, 2016

SRNL-STI-2015-00454, Revision 0 
SRNL-STI-2015-00454

Revision 0

\section{DISCLAIMER}

This work was prepared under an agreement with and funded by the U.S. Government. Neither the U.S. Government or its employees, nor any of its contractors, subcontractors or their employees, makes any express or implied:

1. warranty or assumes any legal liability for the accuracy, completeness, or for the use or results of such use of any information, product, or process disclosed; or

2. representation that such use or results of such use would not infringe privately owned rights; or

3. endorsement or recommendation of any specifically identified commercial product, process, or service.

Any views and opinions of authors expressed in this work do not necessarily state or reflect those of the United States Government, or its contractors, or subcontractors.

\section{Printed in the United States of America \\ Prepared for U.S. Department of Energy}


Keywords: Instrumentation, Process Monitoring, Calibration

Retention: Permanent

\title{
Spectrophotometers for Plutonium Monitoring in HB-Line
}

\author{
R.J. Lascola \\ P.E. O'Rourke \\ E.A. Kyser \\ D.M. Immel \\ J.R. Plummer \\ E.V. Evans
}

February 12, 2016

Prepared in conjunction with work accomplished under contract number DE-AC09-08SR22470 with the U.S. Department of Energy (DOE) Office of Environmental Management (EM).

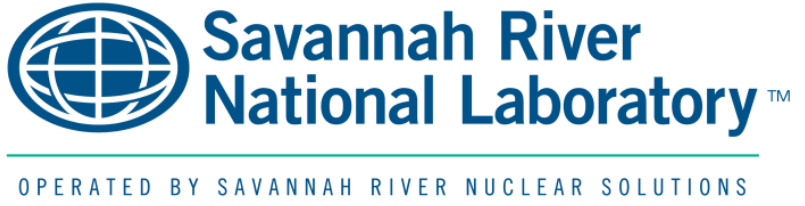




\section{REVIEWS AND APPROVALS}

\section{AUTHORS:}

R.J. Lascola, Science \& Technology Directorate, Analytical Development Date

P.E. O'Rourke, Science \& Technology Directorate, Analytical Development Date

TECHNICAL REVIEW:

L.E. Roy, E\&CPT, Reviewed per E7 2.60 Date APPROVAL:

M.J. Barnes, Manager Date

Science \& Technology Directorate, Analytical Development 


\section{ACKNOWLEDGEMENTS}

This work would not have been possible without the following contributions. Wanda Matthews assisted with glove box operations during standards measurements and column runs. Michael Holland of SRNL's Analytical Laboratories organized characterization of the stock solutions used to make the calibration standards. Gary Dobos streamlined the procurement process for instrument components and absorbance standards. Lindsay Roy, in addition to providing technical review of this document, helped us communicate the sometimes esoteric technical details of the project to our customers. Deanna Stimac, Chris Loyal, and James Therrell in HB-Line Engineering helped keep the project on track, facilitated communication between SRNL and HB-Line stakeholders, and provided important insight into process requirements. Ed Sadowski and Jeff Schaade provided critical programmatic support in promoting the benefits of this instrumentation and keeping enthusiasm and facility support of this project at a high level even during the challenges of process startup. 


\section{EXECUTIVE SUMMARY}

SRNL has developed spectrophotometers to replace colorimeters for monitoring plutonium in several locations in HB-Line, specifically the effluent from the NK-1 and NK-3 columns and the raffinate stream. The spectrophotometers have a much-improved measurement accuracy over the range of conditions expected in the AFS-2 processing campaign. The improvements arise from the measurement of absorbance over the entire visible spectrum, rather than at just two wavelengths as is done with the colorimeters. The extra data obtained allows for statistical models that compensate for the spectral changes associated with changes in nitric acid concentration, plutonium disproportionation, and temperature. These factors introduce a large uncertainty into the colorimeter readings, greatly reducing process throughput by requiring low set points to preserve criticality safety. The accuracy of the spectrophotometers for total plutonium measurement depends on acidity and the plutonium concentration, and thus varies at different points of the AFS-2 process. For example, the 1-sigma uncertainty at likely heart to tail cut set points of the elution process is $\sim 2.4 \%$ (not including other uncertainties associated with the DCS loop).

SRNL has assembled commercial, off the shelf equipment and written custom control software to make a spectrophotometer that has accuracy and stability suitable for operation in HB-Line. The instrument is self-referencing for both wavelength and intensity measurements and the performance is confirmed with NIST-traceable absorbance standards. This process verifies that absorbance measurements for a single instrument are accurate and confirms that different instruments will produce identical absorbance spectra within the uncertainty of the absorbance standards. One consequence of this consistency is that a plutonium calibration model derived from spectra measured with one calibrated spectrometer can be used without adjustment on other calibrated spectrometers. The system derives additional accuracy through a spectral classification scheme based on solution acidity and absorbance. The classification specifies a particular plutonium calibration that is customized for a subset of the total acidity and plutonium concentration range. By having to accommodate less spectral variation, the localized calibrations are simpler than a single global calibration.

This report describes the equipment, control software, calibrations for total plutonium and plutonium oxidation state, and qualification studies for the instrument. It also provides a detailed description of the uncertainty analysis, which includes source terms associated with plutonium calibration standards, instrument drift, and inter-instrument variability. Also included are work instructions for instrument, flow cell, and optical fiber setup, work instructions for routine maintenance, and drawings and schematic diagrams. 


\section{TABLE OF CONTENTS}

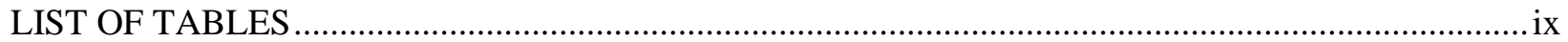

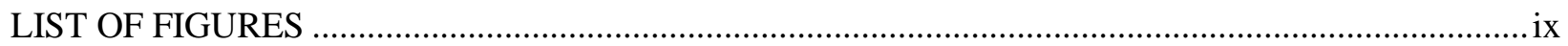

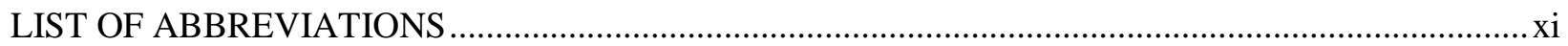

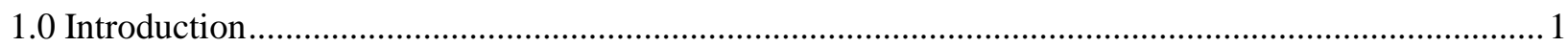

1.1 Chemistry and spectroscopy of Pu process solutions ................................................................... 1

1.2 Limitations of colorimeters, advantages of spectrophotometers ....................................................... 4

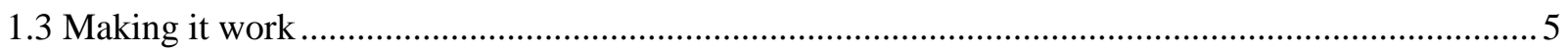

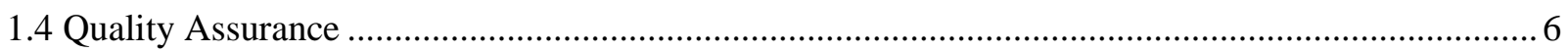

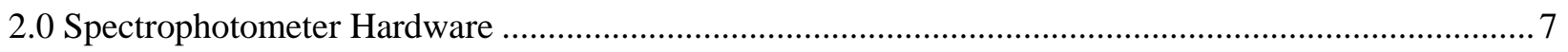

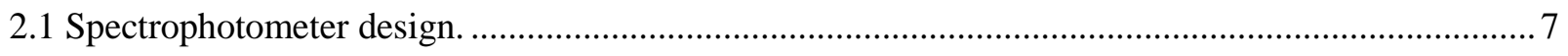

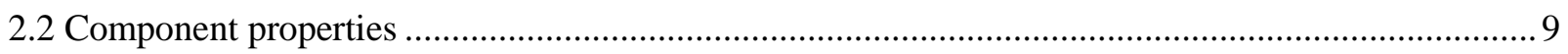

2.3 Relationship to facility and common mode analysis................................................................. 19

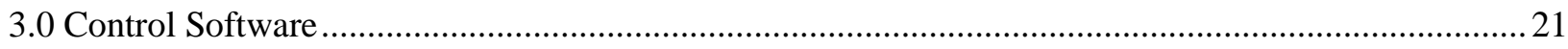

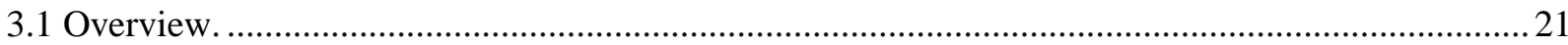

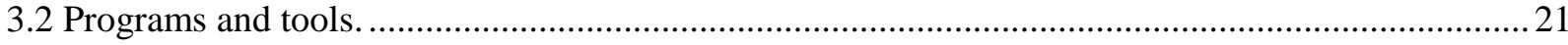

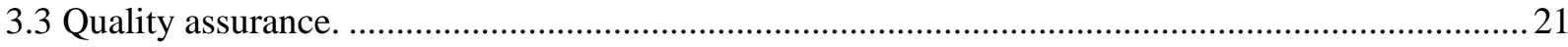

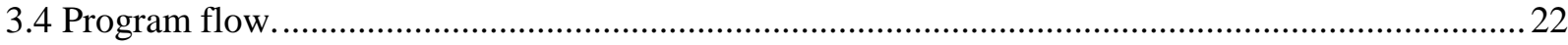

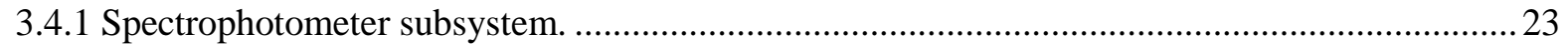

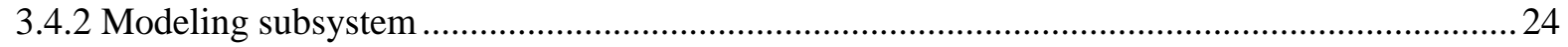

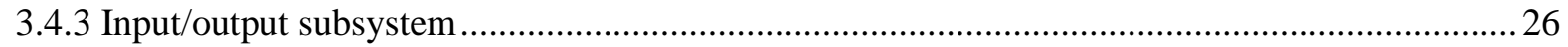

3.4.4 Instrument operation, calibration and troubleshooting ............................................................ 26

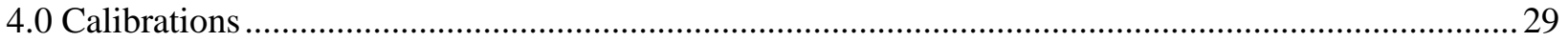

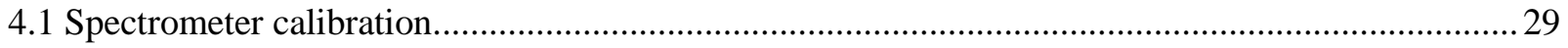

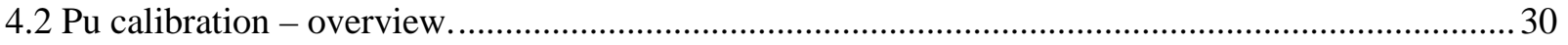

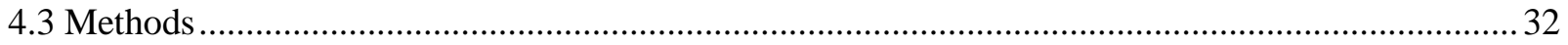

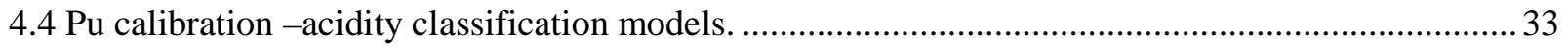

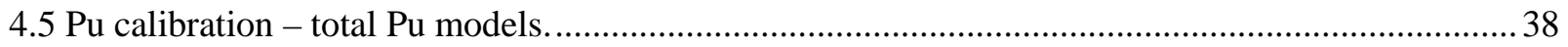

4.6 Pu calibration - Pu oxidation state distribution............................................................................... 46

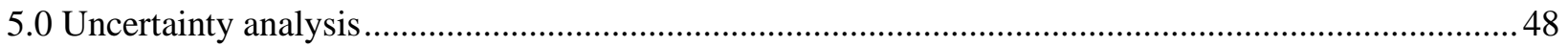

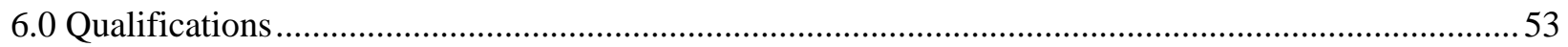

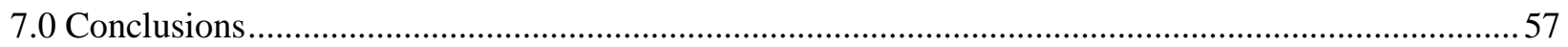

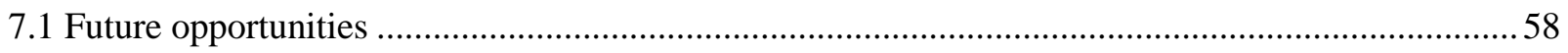

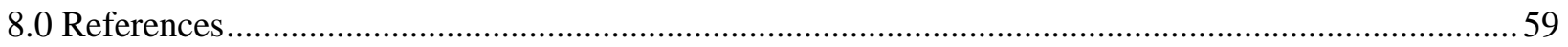


SRNL-STI-2015-00454

Revision 0

<Appendices have been redacted from the distributed document.> 


\section{LIST OF TABLES}

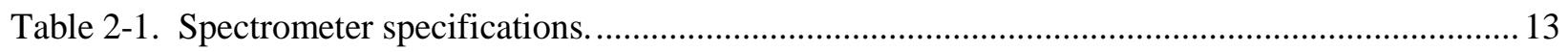

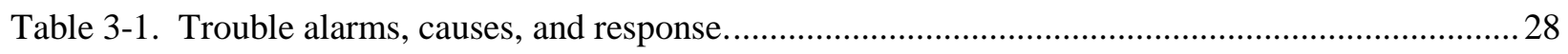

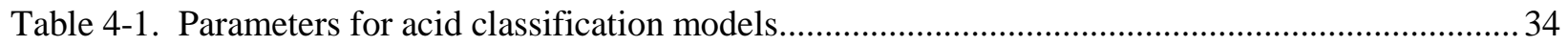

Table 4-2. Total $[\mathrm{Pu}]$ model parameters, 6-9 M acid cases. ............................................................... 39

Table 4-3. Total [Pu] model parameters, 2-6 M acid cases. .................................................................. 39

Table 4-4. Total [Pu] model parameters, 0.3-2 M acid cases................................................................ 40

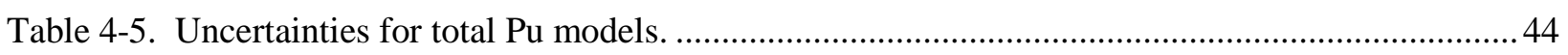

Table 4-6. Inter-instrument variability as a function of total Pu calibration model................................. 46

Table 4-7. Pu oxidation state model parameters................................................................................. 47

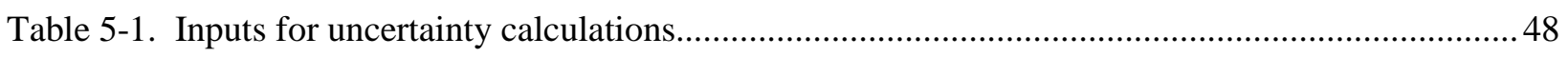

Table 5-2. Total uncertainties for various process conditions. ............................................................... 51

Table 5-3. Association of uncertainties with process steps.................................................................... 52

\section{LIST OF FIGURES}

Figure 1-1. Dependence of the Pu nitrate absorbance spectrum on nitric acid concentration.................... 2

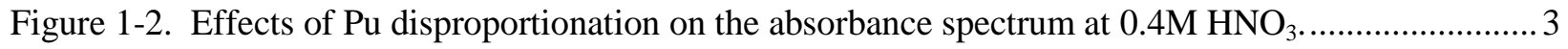

Figure 1-3. Effects of temperature on the Pu absorbance spectrum at $4 \mathrm{M} \mathrm{HNO}_{3}$................................. 3

Figure 2-1. Spectrophotometer process equipment, front view. ........................................................ 8

Figure 2-2. Schematic (top) and photograph (bottom) of spectrophotometer component plate................. 10

Figure 2-3. Effect of modification of tungsten lamp output (top) on measured absorbance spectrum

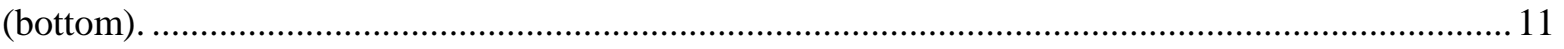

Figure 2-4. Output of xenon flash lamp (top) and expected position of selected xenon atomic emission

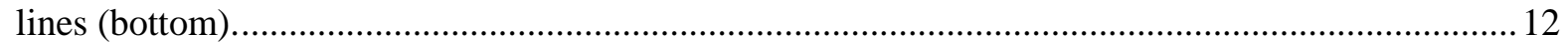

Figure 2-5. Expanded portion of Xe lamp emission, showing multiple pixel points per emission line. .... 14

Figure 2-6. Effect of wavelength calibration error $(\Delta \lambda)$ on Pu prediction............................................... 15

Figure 2-7. Corrected dark current (grey) and observed stray light levels (red/blue)............................. 16

Figure 2-8. Example absorbance spectra of spectrometer calibration standards. .................................... 17 


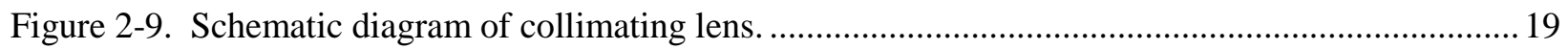

Figure 2-10. Power and communication connections for the spectrophotometers. ................................. 20

Figure 3-1. Top-level schematic for control software operation........................................................... 22

Figure 3-2. Flow diagram for spectrophotometer subsystem.............................................................. 23

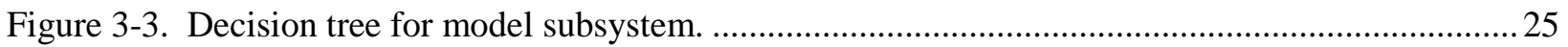

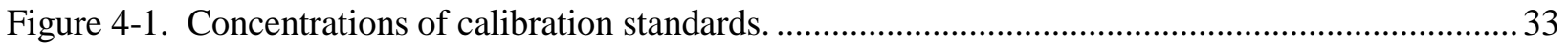

Figure 4-2. Results of low absorbance acid prediction model........................................................... 36

Figure 4-3. Results of medium absorbance acid prediction model......................................................... 37

Figure 4-4. Results of high absorbance acid prediction model........................................................... 37

Figure 4-5. Temperature dependence of acid classification results. ..................................................... 38

Figure 4-6. Residual plots for high acid total plutonium models...................................................... 41

Figure 4-7. Residual plots for medium acid total plutonium models.................................................... 42

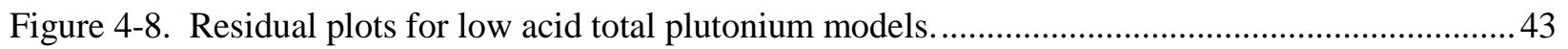

Figure 4-9. Temperature dependence of total Pu model R2Putot11....................................................... 44

Figure 4-10. Relation of $[\mathrm{Pu}]$ to absorbance region for a 1 inch flow cell............................................. 45

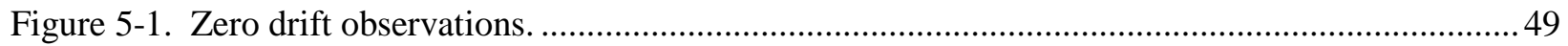

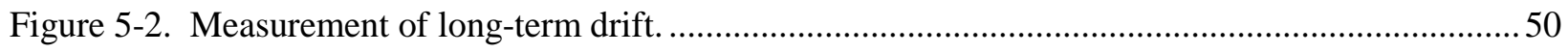

Figure 6-1. Qualification data for Pu standards in cuvettes..............................................................5

Figure 6-2. Results of monitoring two laboratory Pu column elutions using the spectrophotometer......... 55

Figure 6-3. Simulated spectrophotometer response for column elutions with 1” flow cell....................... 56 


\section{LIST OF ABBREVIATIONS}

\begin{tabular}{ll}
$A$ & Absorbance [dimensionless quantity] \\
AFS-2 & Alternate Feed Stock \\
$b$ & Absorption pathlength [centimeters] \\
CLI & Component Location Identifier \\
COTS & Commercial, off-the-shelf \\
DCS & Distributed control system \\
HNO 3 & Nitric acid \\
Ho & Holmium \\
I/O & Input / Output \\
ISO & International Organization for Standardization \\
M & Molarity [moles/liter] \\
M\&TE & Measurement and Test Equipment \\
ND & Neutral Density \\
NGS & Next Generation Spectrophotometer \\
NIST & National Institute for Standards and Technology \\
nm & Nanometers \\
PC(A) & Principal Component (Analysis) \\
PLS & Partial Least-Squares (Analysis) \\
Pu & Plutonium \\
RMS & Root mean square \\
RMSE(C,CV) & Root mean-square error of (Calibration, Cross-Validation) \\
SG & Savitzky-Golay \\
SRNL & Savannah River National Laboratory \\
UPS & Uninterruptible Power Supply \\
$\varepsilon$ & Molar absorptivity [liter/moles-centimeter] \\
& \\
\hline &
\end{tabular}




\subsection{Introduction}

The AFS-2 plutonium (Pu) processing campaign relies on several process monitors to automatically start and stop collection of the eluent from the NK-1 and NK-3 ion exchange columns and to protect criticality limits at the H Canyon raffinate receiving tank. [1] The operating principle for the instruments, which are referred to as "colorimeters", is based on a linear relationship between absorbances at two specific wavelengths (colors of light) and Pu concentration in the process solution. It has been recognized that several properties of the process solution cause the absorbance/concentration relationship to deviate from linearity. [2] Further, the colorimeters are prone to drift in the period between calibrations. These factors result in a very large uncertainty in the colorimeter readings, which significantly impacts processing operations by requiring greater dilution of the solution collected from the columns and a much lower tolerance for Pu in the raffinate stream. The effect on operations is so strong as to merit the development of alternative instrumentation. Spectrophotometers measure absorbances at multiple wavelengths simultaneously. This property allows the application of more sophisticated mathematical methods to establish accurate determination of Pu concentrations over the wide range of AFS-2 process conditions. It also allows for real-time diagnostics that can better correct for instrument drift. As a result, the uncertainty of Pu measurements with spectrophotometers will be much better than with colorimeters.

This report describes the development and properties of Next Generation Spectrophotometers (NGS) as replacement process $\mathrm{Pu}$ monitors. Functional and performance requirements for the instruments are described in the Task Technical Request NMMD-HTS-2013-3249, Rev. 1, which is included as Appendix A. The remainder of the introduction will summarize the properties of Pu absorption spectroscopy and instrument design that promote the use of spectrophotometers for this application. Sections 2 and 3 will provide details on the design and assembly of the instrument hardware and control software, respectively. Section 4 will discuss the calibration of the spectrophotometers. Section 5 will discuss various source terms associated with the uncertainty of the reported total plutonium concentrations. Section 6 will demonstrate the qualification of the instruments. Additional appendices will cover technical details, including instrument drawings and work instructions for field operation, calibration, and maintenance.

\subsection{Chemistry and spectroscopy of Pu process solutions}

The relationship between absorbance $(A)$ and concentration $(c)$ is given by the Beer-Lambert Law,

$$
A(\lambda)=-\log _{10}\left[I(\lambda) / I_{0}(\lambda)\right]=\sum_{i}\left[\varepsilon_{i}(\lambda) \cdot b \cdot c_{i}\right]
$$

where $A$ is the logarithm of the ratio of the incident $\left(I_{0}\right)$ and transmitted $(I)$ light intensities, $\varepsilon_{i}(\lambda)$ is the wavelength $(\lambda)$-dependent molar absorptivity of the absorbing species $i$, and $b$ is the distance that the light travels through the solution ("pathlength"). A key point of this expression is that the total absorbance is equal to the sum of the absorbances of the $i$ individual species in the sample, weighted by the concentrations of those species. Assumptions about the solution composition can affect whether or not the solution appears to obey this law.

For example, the quantity of interest for process control and safety is the total Pu concentration $\left(\left[\mathrm{Pu}_{\text {total }}\right]\right)$, but in the process solution $\mathrm{Pu}$ can exist in several different forms, each with a distinct absorbance spectrum. For example, there may be several different Pu nitrate species of the form $\mathrm{Pu}\left(\mathrm{NO}_{3}\right)_{\mathrm{n}}{ }^{4-\mathrm{n}}(\mathrm{n}=0,1$, $2,4,6)$. [3-5] The relative quantities of each are determined by the nitric acid concentration, which varies from $0.3-9 \mathrm{M}^{1}{ }^{1}$ The measured absorbance is the sum of the contributions of the individual nitrate species. The absorbance spectrum changes with acidity as shown in Figure 1-1. In this figure, $\left[\mathrm{Pu}_{\text {total }}\right]$ is the same for each spectrum, but the height and shape of the peaks in the spectrum change as increasing

\footnotetext{
${ }^{1}$ For the range of $[\mathrm{Pu}]$ in the process solutions, the Pu nitrate distribution is not dependent on $\left[\mathrm{Pu}_{\text {total }}\right]$.
} 
acidity favors the formation of more highly nitrated complexes. It is apparent that there is no single wavelength for which absorbance remains constant across the specified acid range.

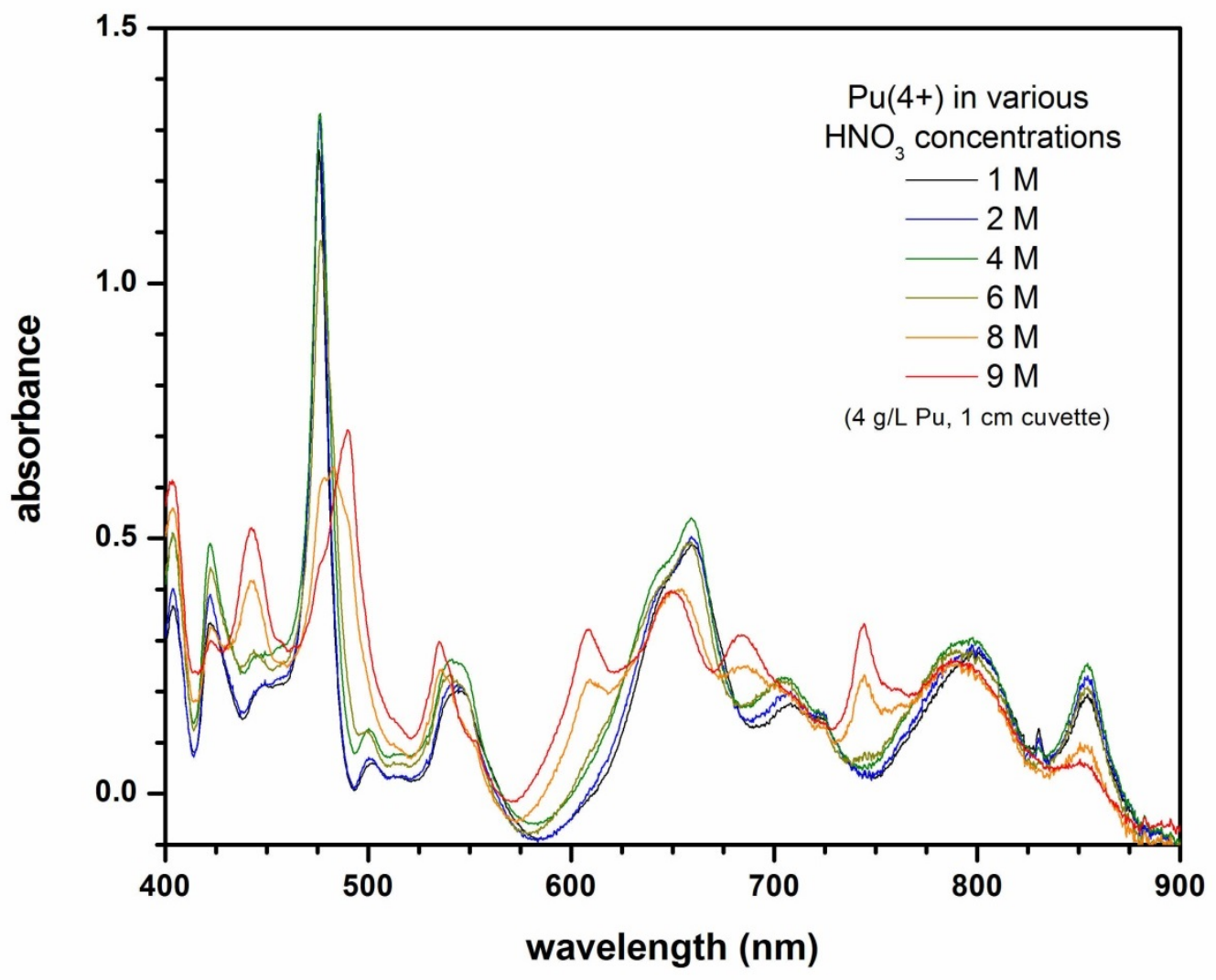

Figure 1-1. Dependence of the Pu nitrate absorbance spectrum on nitric acid concentration.

The process flowsheet is designed for $\mathrm{Pu}$ to be in the 4+ oxidation state. However, for $\left[\mathrm{HNO}_{3}\right]<2 \mathrm{M}$, $\mathrm{Pu}^{4+}$ disproportionates according to the reaction

$$
3 \mathrm{Pu}^{4+} \leftrightarrow 2 \mathrm{Pu}^{3+}+\mathrm{Pu}^{6+} .
$$

This reaction is favored by lower acidities and larger [Pu], and has been demonstrated to be feasible at the conditions for column elution. [6] For monitoring purposes, a complication arises from the fact that the absorption spectra of the $\mathrm{Pu}$ nitrates depend on the oxidation state of $\mathrm{Pu}$. The spectral effects of disproportionation are shown in Figure 1-2. For the spectra shown here, $\left[\mathrm{Pu}_{\text {total }}\right]$ and $\left[\mathrm{HNO}_{3}\right]$ are constant, and disproportionation is increasing with time. The growth of several peaks due to the increased presence of the $3+$ and $6+$ oxidation states is apparent, with a proportional decrease of peaks attributable to the 4+ oxidation state.

Another factor which influences absorption measurements is solution temperature, which is expected to vary between $20-45^{\circ} \mathrm{C}$. As shown in Figure 1-3, for some spectrum peaks (475 nm), temperature increases mimic the effect of increasing nitric acid concentration. However, other peaks $(650 \mathrm{~nm})$ do not shift similarly, and thus temperature must be considered as a separate influence on the absorption spectrum. 


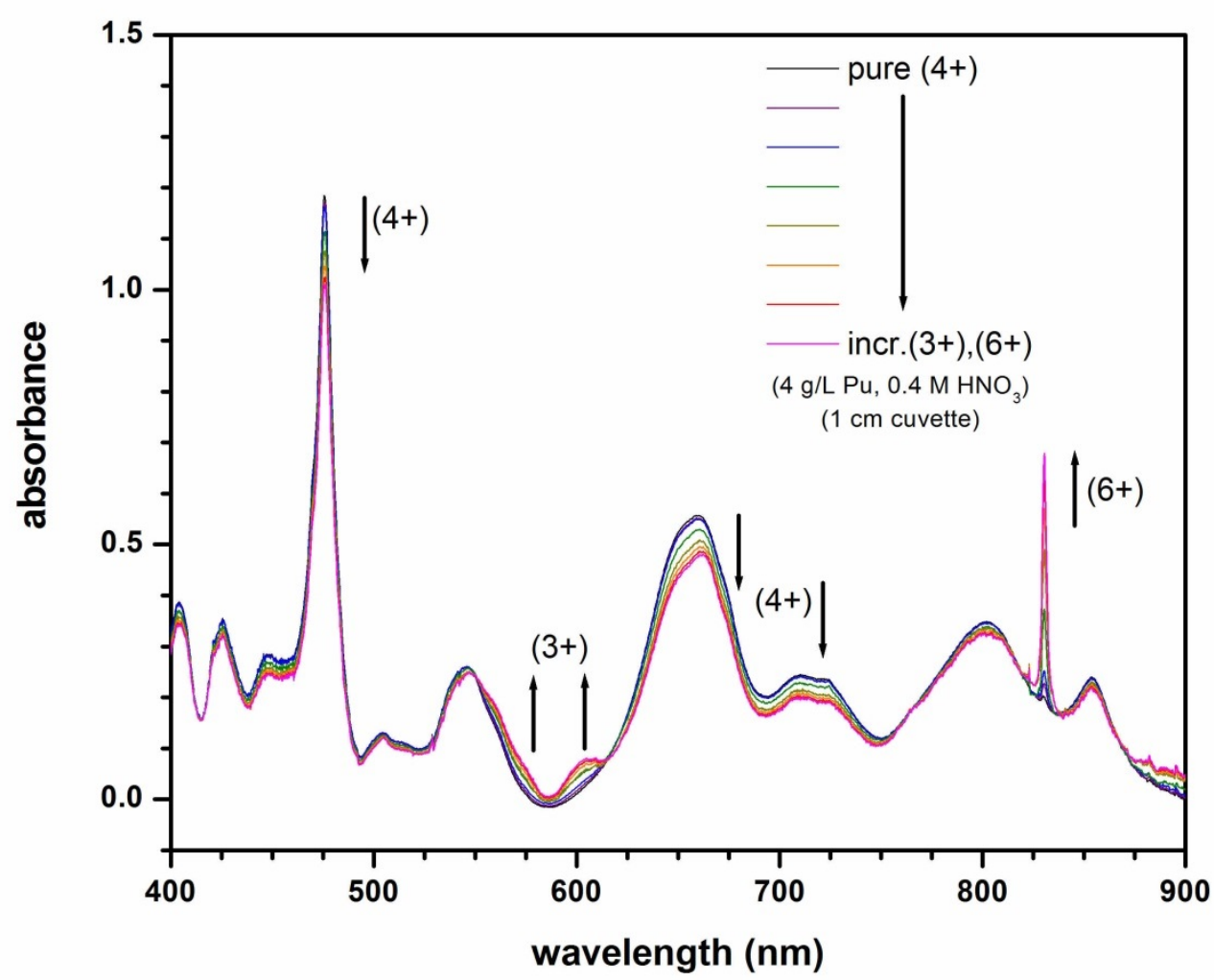

Figure 1-2. Effects of Pu disproportionation on the absorbance spectrum at $0.4 \mathrm{M} \mathrm{HNO}$.

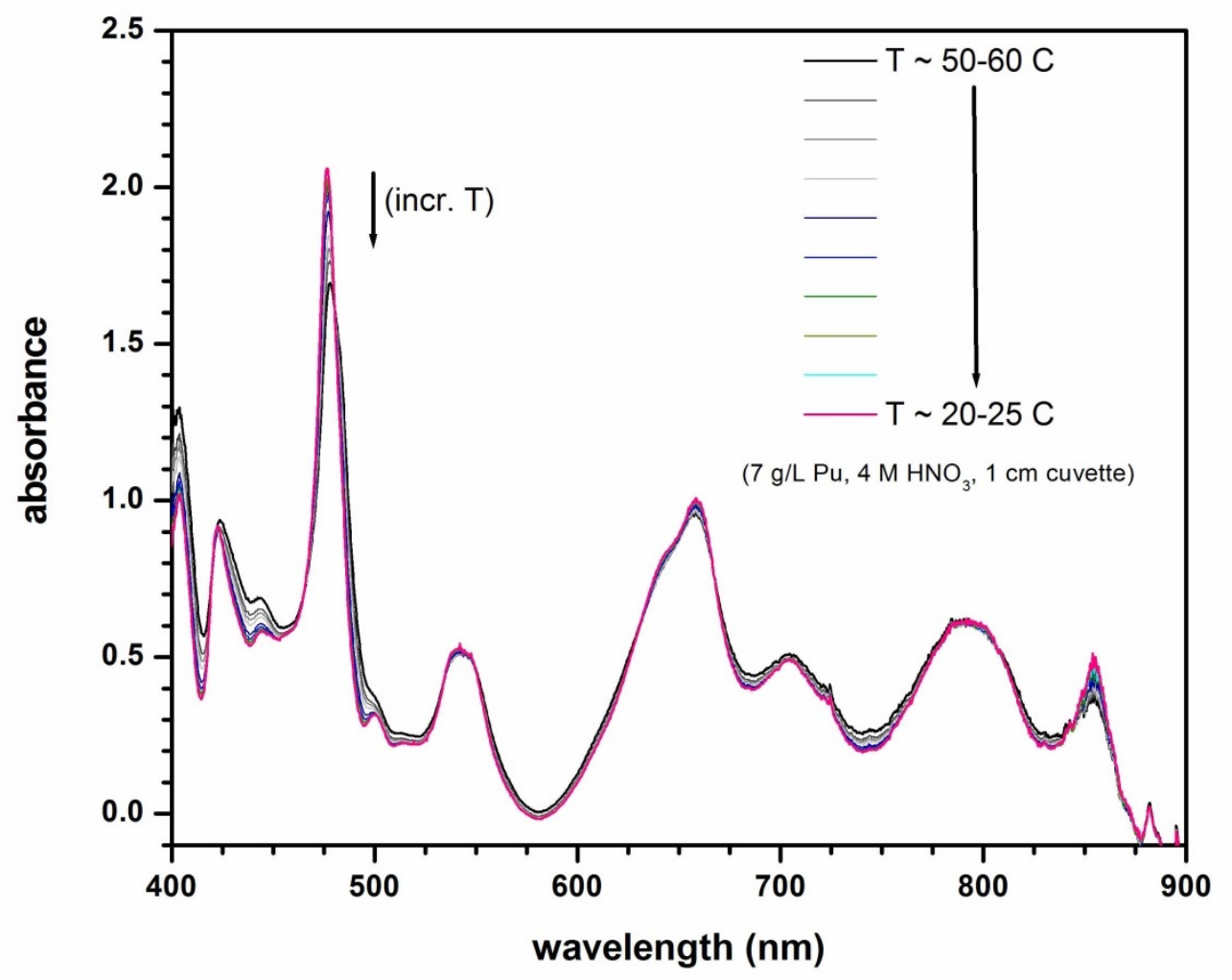

Figure 1-3. Effects of temperature on the Pu absorbance spectrum at $4 \mathrm{M} \mathrm{HNO}_{3}$. 


\subsection{Limitations of colorimeters, advantages of spectrophotometers}

The operating principle of the colorimeter is to measure solution absorbance at two wavelengths, designated "measure" and "reference". The measure wavelength is the wavelength of peak absorbance of the analyte, and the reference wavelength is chosen where the analyte does not absorb any light. These quantities represent $I$ and $I_{0}$ in Equation 1 . Separate detectors measure each quantity simultaneously, with the wavelength response of the detectors defined by bandpass filters which transmit a narrow section of the spectrum at the desired wavelength. The limitations of colorimeters for $\mathrm{Pu}$ measurements are apparent from the above figures. There is no wavelength for which total absorbance remains constant across the span of acidities in the process. There also is no wavelength at which Pu does not absorb, and thus one cannot choose an invariant reference. The wavelengths chosen for the colorimeters (510 \pm 10 $\mathrm{nm}$ for the measure and $589 \pm 10 \mathrm{~nm}$ for the reference) have the lowest variance ( 10-15\%) across the acid range for commercially available bandpass filters.

A more critical impact comes from disproportionation. The colorimeters are calibrated with the assumption that all $\mathrm{Pu}$ is present as $\mathrm{Pu}^{4+}$, and so loss of $\mathrm{Pu}^{4+}$ to the other oxidation states manifests as a negative bias in the colorimeter reading. The effect is magnified by the absorption due to $\mathrm{Pu}^{3+}$, which has a greater absorbance at the $\mathrm{Pu}^{4+}$ reference wavelength $(589 \mathrm{~nm})$ than at the $\mathrm{Pu}^{4+}$ measure wavelength (510 $\mathrm{nm})$. Thus, the presence of $\mathrm{Pu}^{3+}$ also constitutes a negative bias for colorimeter reading. We have previously estimated that for a feasible amount of disproportionation (10\% during the tail cut of column elution), colorimeter bias could be as large as -37\%. [2]

It would be hypothetically possible to correct the colorimeter reading for these factors at the distributed control system (DCS) if they were externally measured in real time. However, neither an acidity nor a disproportionation sensor is in place in the process, and thus the most conservative scenarios must be considered when calculating control limits for the process.

There are other limitations associated with the colorimeters which are independent of the Pu analyte. The two-detector system assumes that changes in lamp output will affect both detectors equally. However, this is not likely to be true for colorimeters which use tungsten filament lamps. As the lamp ages, it requires having a higher potential applied to it to maintain the same overall output intensity. The color of the lamp output changes when this occurs, leading to a drift as the intensities at the two detectors do not change proportionally. The optical properties of the filters may change over time, also altering the response. The response to these factors has been to increase the frequency of recalibration, but even within these shorter time periods, there are additional contributions to the overall uncertainty.

One must also consider that irregularities in the flowing solution can influence the colorimeter reading. Turbulence from entrained air can scatter light, reducing transmission and introducing extra variability to the measurement. Nuisance alarms sometimes occur when the variability leads to a temporary false spike in the colorimeter reading. The spikes occur because the colorimeter, by measuring at only two wavelengths, cannot distinguish between a true change in $\left[\mathrm{Pu}_{\text {total }}\right]$ and the baseline shift associated with turbulence.

An alternate way of expressing the colorimeter limitations suggests how the spectrophotometer can address those issues. Each factor that has been described - multiple $\mathrm{Pu}$ nitrate species, multiple $\mathrm{Pu}$ oxidation states, solution temperature variations, lamp variations, process irregularities - is an independent contributor to the overall absorbance that is measured, in addition to the desired factor, total $\mathrm{Pu}$ concentration. However, the colorimeter only measures two variables, the intensities on the measure and reference detectors. There is an information deficit, from which the colorimeter will not be able to distinguish the source of a signal change. This results in large uncertainties, since it must be assumed that the detected signal arises solely due to the desired factor. 
The advantage of the spectrophotometer is that by measuring absorbance at multiple wavelengths, it acquires much more information that can be used to distinguish the various sources of signal changes. Elements of the instrument design - hardware, control software, and data analysis - are chosen to maximize this ability. For example, the spectrophotometer contains two spectrometers ${ }^{2}$, one of which is dedicated to monitoring the intensity spectrum. The readings on that spectrometer are used to correct the measured intensity of light transmitted by the solution. The net effect is that lamp source changes produce negligible instrument drift. A second example arises from the simultaneous measurement of absorbances at multiple wavelengths. In contrast to the colorimeter, there are more measured variables than sources of signal variation. Mathematical expressions can be generated that permit the calculation of $\left[\mathrm{Pu}_{\text {total }}\right]$ despite the spectral effects demonstrated in Figure 1-1 through Figure 1-3. Other examples are provided below. By obtaining more accurate absorbance data, and performing more accurate analysis of the data, the spectrophotometer can make a more accurate $\left[\mathrm{Pu}_{\text {total }}\right]$ reading than can the colorimeter over a wider range of process conditions.

\subsection{Making it work}

The above discussion does not imply that colorimeters are generally poor instruments. In the right situation, such as a limited process chemistry and simple spectroscopy, the instruments can be used in an off-the-shelf configuration with satisfactory results. [7] Even as the sample becomes more complex, modifications can extend the scope of the colorimeter. For example, in HB-Line the instruments are proficient at detecting the onset of the heart cut during column elution, despite the rapid change in solution acidity that accompanies the elution front. This was originally achieved by using custom bandpass filters to minimize response variation, and by developing custom optical fiber bundles to suppress the low light nuisance alarms that would otherwise occur during the middle of the heart cut, when the flow cell would not transmit light.

A similar development principle applies to spectrophotometers. Some performance requirements imposed by the operating conditions in HB-Line require development work to obtain desired performance from off-the-shelf instruments. In this case, the modifications resulted in the development of a new instrument constructed from commercially available components and incorporating custom-built control software. Similarly, the Pu chemistry in the process solution is too complex to apply normal calibration methods in a rote fashion. The difference between the use of colorimeters and spectrophotometers in this application is that no amount of modification could make the colorimeters work well for raffinate and heart/tail cut monitoring, whereas the enhanced spectrometer capabilities are more than sufficient for these purposes.

Operational parameters peculiar to HB-Line, and instrument modifications made to address them, are summarized below. Detailed discussions, with data supporting the claims made, can be found in the appropriate sections (hardware, control software, calibration).

- Field validation of instrument calibration for $\mathrm{Pu}$. There is no capability to pour Pu standard solutions into the spectrophotometer flow cells in HB-Line to calibrate/validate instrument performance. We have developed a scheme whereby the absolute accuracy of the instrument's absorbance measurements is confirmed by the measurement of NIST-traceable general absorbance standards. The spectrophotometers were validated with these standards before the $\mathrm{Pu}$ calibrations were made. As long as the instruments are periodically validated by remeasurement of the absorbance standards, the Pu-specific calibrations can be assumed to apply. This provides the means to continually monitor and confirm the instrument performance and calibration without uninstalling the spectrometers, as is currently done with the colorimeters.

\footnotetext{
${ }^{2}$ The spectrometer is the component of the spectrophotometer where the different wavelengths in the light source are separated and detected.
} 
- Combined light sources. The outputs of a tungsten and a xenon arc lamp are combined into a single light source. The combination allows more even intensity distribution over the full wavelength range. The xenon arc lamp also provides internal wavelength standards that are used to adjust the intrinsic instrument calibration (intensity vs. wavelength) in real time, improving stability and accuracy. This arrangement is a unique combination of commercially available light sources.

- Spectrometer characterization and the "virtual instrument". In addition to wavelength, the intensity response of each array detector in the spectrophotometer is monitored and corrected for the effects of stray light, dark current, and readout noise. This expands the accuracy of the instrument when measuring both small and large absorbances. The combined wavelength and intensity corrections are robust enough to make the responses from different spectrophotometers look as if they came from the same instrument. Such a "virtual instrument" has several advantages. Spare instruments can be installed without loss of quality. New calibrations, for example to accommodate a change in the process conditions outside the original scope, can be developed in the laboratory and ported to all spectrometers with high confidence and with minimal impact on existing operations.

- Spectral classification based on absorbance. The range of $\left[\mathrm{Pu}_{\text {total }}\right]$ which can be measured can be greatly increased (by roughly a factor of 3) by classifying the spectra based on the absorbance at several key wavelengths. As concentration increases, the absorbance at those wavelengths will eventually exceed the threshold for spectrometer accuracy. These wavelengths can be systematically excluded from the data analysis when this occurs.

- Spectral classification based on nitric acid concentration. The complexity of the $\left[\mathrm{Pu}_{\text {total }}\right]$ calibration increases linearly with the number of confounding factors. Although there is a large amount of information available in a full absorbance spectrum, finite signal quality limits how many confounding factors can be handled. However, samples and more accurate models can be made if they are restricted to a subset of the total range of process conditions. An effective way to do this is to divide the acidity range into three subsets. Within each subset, there will be fewer $\mathrm{Pu}$ nitrate species than in the entire acidity range. The acidity subsets can also be chosen so that disproportionation only occurs in one subset. Furthermore, the acidity can be accurately determined from the spectra, so no additional data (or instruments) are required. Neither the absorbance nor acidity classification schemes are features of commercially available analysis software, and are a custom feature of the control software written for this instrument.

- Diagnostics. Issues that arise during operation often have spectral signatures which can be monitored. Detection of these signatures reduces the reporting of dubious results and provides a means for indicating specific alarms. Conditions which can be reported include turbulence in the flow cell, failure of a light source, or excursion of conditions outside the range covered in the calibration. These diagnostics have been included in the control software.

\subsection{Quality Assurance}

Requirements for performing reviews of technical reports and the extent of review are established in manual E7 2.60. SRNL documents the extent and type of review using the SRNL Technical Report Design Checklist contained in WSRC-IM-2002-00011, Rev. 2. 


\subsection{Spectrophotometer Hardware}

There has been a substantial amount of development associated with the hardware and control software for the spectrophotometers. This work was undertaken with the realization that commercially available, off-the-shelf (COTS) instruments would not meet the performance requirements for successful field operation in HB-Line. Those requirements are based on operational experience with the colorimeters in HB-Line and the colorimeters and spectrophotometer system in H Canyon. Performance goals include the following:

- Reduced instrument maintenance. A concern for the colorimeters is the need for frequent maintenance. Rapid lamp aging and a high sensitivity of instrument reading to the changing lamp output (e.g. instrument drift) has led to monthly field calibrations. The calibrations require significant commitment of worker resources and dedicated down time. A longer period of time ( $\geq 1$ year) between field calibrations is preferred. To achieve this goal requires more stable light sources and reduced sensitivity to lamp changes between calibrations. The instrument has also been designed so that maintenance and calibration can be performed with stepwise procedures in the field.

- Improved operation. The H Canyon spectrophotometers required a dedicated startup procedure. The new instruments behave like the colorimeters in that they will start running when powered on. The new instruments are configured to ensure compatibility with existing equipment in the facility, including the DCS, fiber optics, and flow cells, while supporting future upgrades. The spectrophotometers will also have a more reliable check filter mechanism than is available for the raffinate colorimeter.

- Improved accuracy. Improvements in lamps and spectrometers lead to more accurate absorbance measurements, and ultimately better Pu readings. Both wavelength and intensity are calibrated in real time.

- Instrument interchangeability. The steps made to improve the accuracy of one spectrophotometer can be applied to all spectrophotometers of this design. Doing so enhances the degree to which the instruments are interchangeable. Such a situation provides several advantages. Calibrations made on one instrument (for example dedicated to a laboratory) can be transferred to instruments in the field with minimal loss of accuracy. New components, or even entire units, can be installed and expected to work immediately. Likewise, new instruments installed at different locations (on-site or elsewhere) could use the same calibrations, so long as they are appropriate for the process conditions.

- Reasonable cost. While there has been substantial investment in development, the cost of the individual instruments is comparable to the list price for COTS colorimeters and spectrometers. Improved instrument performance with a reasonable cost for additional instruments and compatibility with existing optical fibers and flow cells leads to the possibility that these units can serve as replacements for the existing but aging colorimeters and spectrophotometers in $\mathrm{H}$ Canyon. These instruments would also be suitable for new monitoring applications elsewhere on site. The spectrophotometers can be readily calibrated for detection of $\mathrm{U}, \mathrm{Np}$, and other species that absorb light in the ultraviolet-visible-near infrared region.

\subsection{Spectrophotometer design.}

An overview of the hardware configurations of the instruments are provided here. Detailed information, such as drawings and component lists, are provided in Appendix G. Halogen and Xe flash lamp replacement instructions are given in Appendices D and E, respectively. Instructions for spectrometer setup and diagnostic evaluation are givenin Appendix F. 
A top-level schematic of the equipment which will be installed in HB-Line Phase II Control Room is shown in Figure 2-1, along with a photograph of the equipment. All components are mounted inside a cabinet with a 19" rack. The cabinet will hold three spectrophotometer systems, with room reserved for a fourth. Each system provided will be a direct replacement for the existing colorimeters (NK-1, NK-3, and raffinate). Each spectrophotometer is mounted on an aluminum plate on a sliding platform which can be accessed for maintenance and calibration. A detailed description of the components on the plate will be presented below. Each spectrophotometer has a dedicated display panel which has a digital display of $\left[\mathrm{Pu}_{\text {total }}\right]$, in units of $\mathrm{g} / \mathrm{L}$, and several alarm lights. Red lights labeled "Xe fault" and "HT fault" are illuminated in cases when the intensity of the xenon flash lamp or tungsten-halogen lamp, respectively, is determined to be deficient. A red light labeled "Trouble" will illuminate along with the lamp fault lights, and will also illuminate by itself when the instrument is taken off line or there are other error conditions. These conditions are determined in the course of obtaining and evaluating the absorption spectrum. A yellow light labeled "Confidence" indicates high fitting residuals, which can arise from turbulence in the flow stream or spectral interferents. The display panel has USB and ethernet ports to allow for installing software upgrades (if necessary) and recovering data files. There is also a toggle switch that is used to take the spectrometer offline for diagnostic and calibration purposes (indicated by a blue light). The switch also controls activation of the check filter mechanism. The spectrophotometer/display panel pairs are sequentially mounted in the lower two-thirds of the cabinet. ${ }^{\text {a }}$ There is room for a fourth spectrometer to be put into the rack.

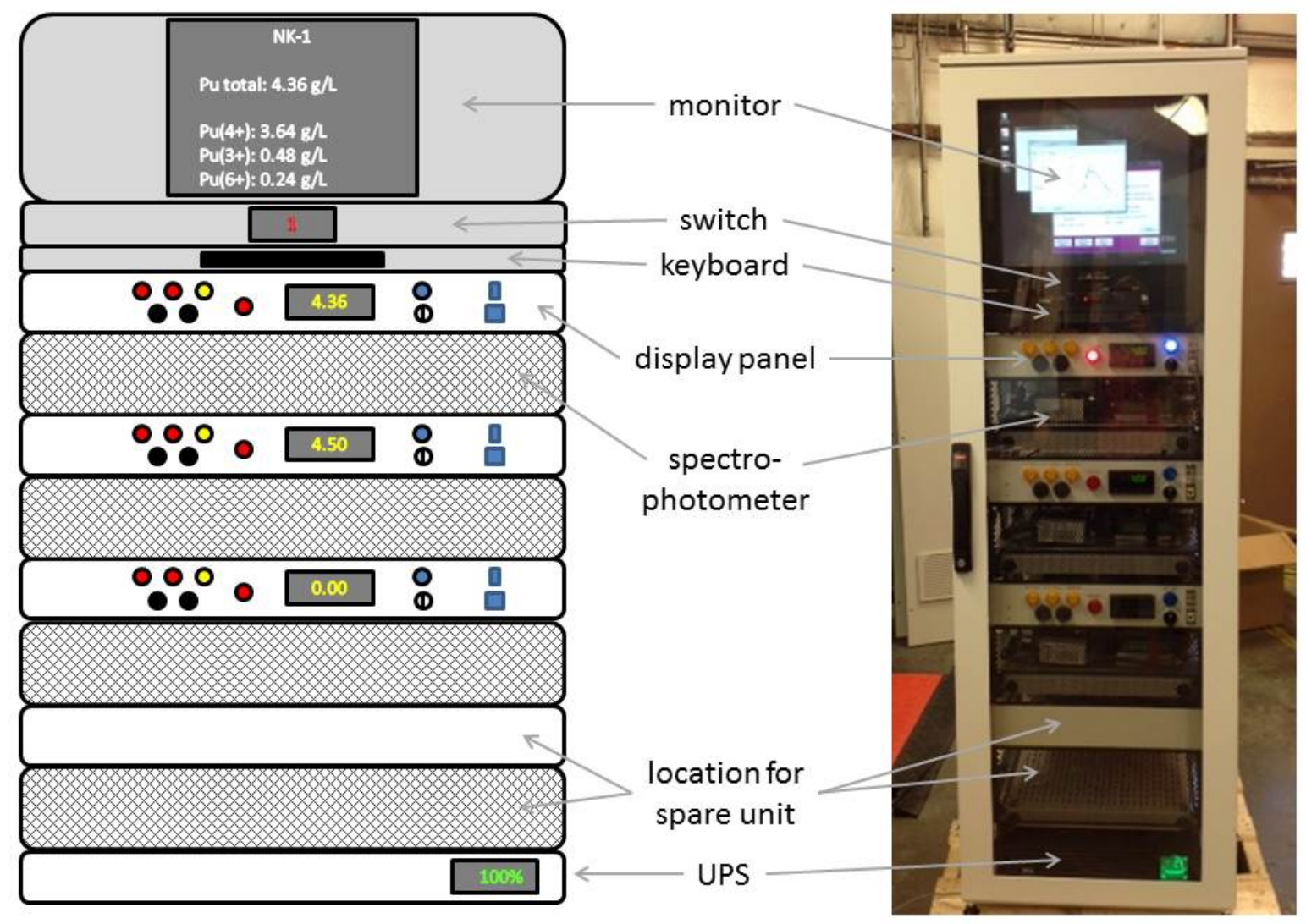

Figure 2-1. Spectrophotometer process equipment, front view.

\footnotetext{
${ }^{\mathrm{a}}$ The instruments are identified as NGS-1 (NK-1 replacement), NGS-2 (NK-2 replacement), and NGS-3 (raffinate replacement).
} 
The top third of the cabinet holds a keyboard and flat-screen monitor, which can be switched between the spectrophotometers. The switching unit also has an ethernet port which may be used to connect a printer in the future. The processing units for each spectrometer are mounted on a shelf inside the cabinet, behind the monitor. Facility optical fibers, which go to the flow cells, enter the right-hand side of the cabinet (as seen from the front), where they are connected to the internal fiber connectors. The cabinet has a clear door to limit access to the system while allowing observation of the instrument displays. On the rear of the cabinet, there are two half-height doors. On the inside of each door, there are two I/O stations. Each station has the capacity for three 4-20 mA analog output modules (currently, only one is in use for reporting $\left[\mathrm{Pu}_{\text {total }}\right]$ ) and two digital output modules (for reporting a trouble alarm and an online status). The I/O is not configured to receive signals from the DCS. An uninterruptible power supply / power conditioner unit is mounted at the bottom of the cabinet. A fan is mounted on the top of the cabinet, drawing hot air from the processing units and monitor away from the spectrometer modules below.

A schematic drawing and photograph of the spectrophotometer plate are shown in Figure 2-2. The spectrophotometer has two light sources, a tungsten-halogen lamp and a xenon flash lamp. Collimated beams of light from each lamp are combined in a 50/50 beamsplitter, which contains a window that is half reflective and half transmissive. To reach the beamsplitter, each beam passes through an adjustable, lockable iris that regulates the intensity. There are two identical beams of light which exit the beamsplitter, each containing half the intensity from each lamp. One beam (going upward in the diagram) passes through a cuvette holder and check filter slot and is coupled into an optical fiber leading to the process flow cell. The check filter slot accepts the check filter when it is activated. The cuvette holder accepts other absorbance standards which are used to verify the calibration of the instrument. Under normal operation, both the slot and holder are empty. The second beam of light exiting the beamsplitter (going leftward in the diagram) is coupled into a fiber and, after passing through another iris, is directly connected to the reference spectrometer. The iris balances light levels between the reference and sample light paths. Light returning from the flow cell is coupled into the sample spectrometer. The intensity spectra on both spectrometers are communicated to the processing unit through the communications (“comms") port. The power supply rail holds several ac/dc converters to supply the spectrometers, lamps, "comms" port, and check filter mechanism.

\subsection{Component properties}

Light sources. The combination of the xenon and tungsten-halogen lamps provides several advantages for stable, long term operation. The lamps have complementary output ranges, with the tungsten lamp dominant for $\lambda>500 \mathrm{~nm}$, and together provide reasonably consistent intensity over the wavelengths necessary for actinide absorbance spectroscopy. Both lamps have long lifetimes (many thousands of hours) and should remain operational for well over a year per manufacturer specifications. The lifetimes support a one-year replacement schedule that is consistent with other facility maintenance protocols. Any degradation that does take place will affect both the reference and sample channels equally, and will be compensated in data processing. This feature greatly reduces instrument drift compared to the colorimeters. This is demonstrated in Figure 2-3, which shows the absorbance spectra obtained in cases when the tungsten lamp intensity is normal (left), reduced by $~ 2 / 3 r d s$ across all wavelengths (middle), and passed through a blue filter (right). The intensity reduction and change in wavelength output induced here are far greater than would be expected during a year's use. In particular, the changing wavelength distribution would cause a severe drift in colorimeter reading, due to the differential changes at the measure and reference wavelengths. However, the absorbance spectra obtained here remain very close to zero, and translate to almost no change in the total Pu reading. 

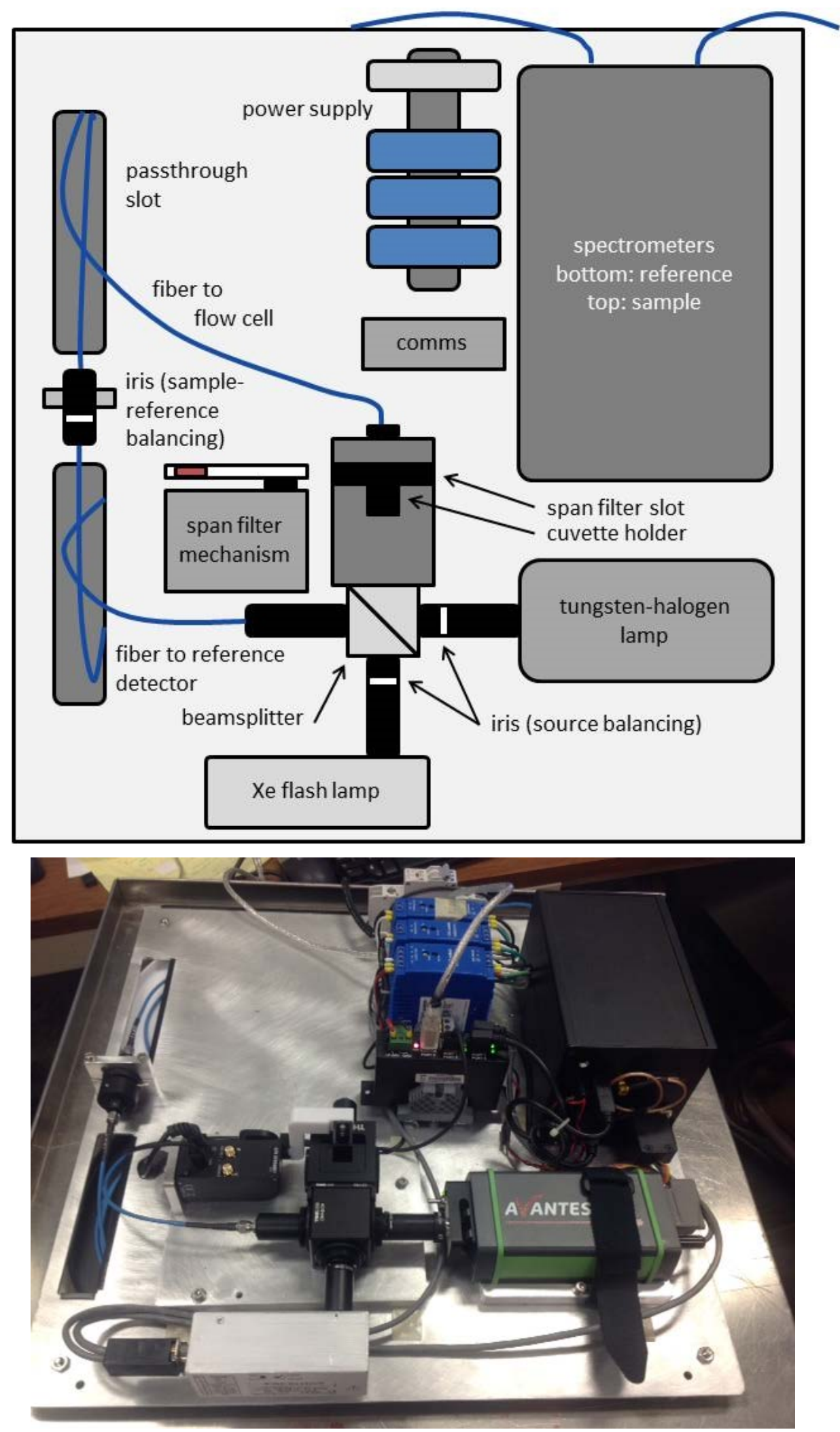

Figure 2-2. Schematic (top) and photograph (bottom) of spectrophotometer component plate. 

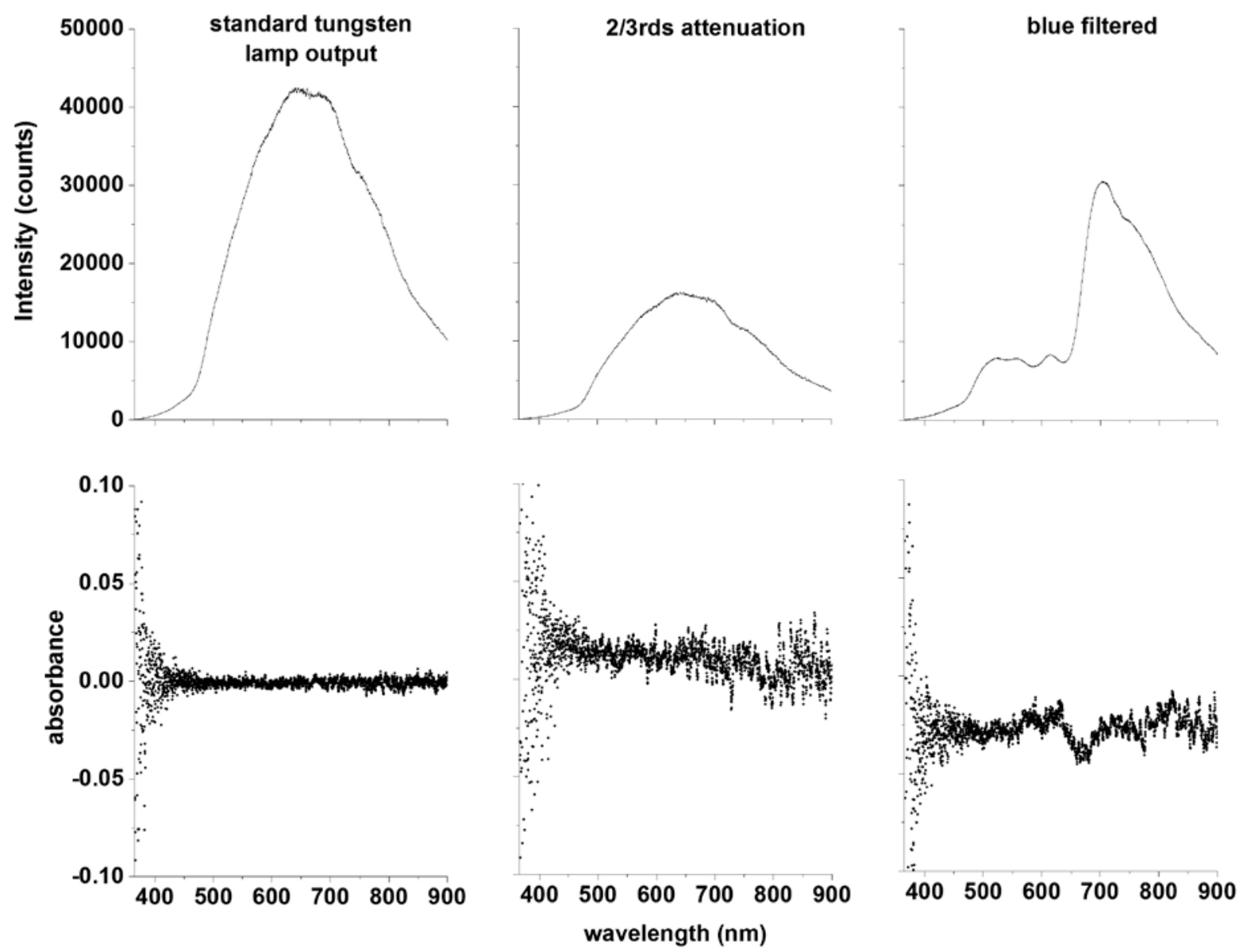

Figure 2-3. Effect of modification of tungsten lamp output (top) on measured absorbance spectrum (bottom).

A further advantage of the Xe flash lamp is that its output contains a number of sharp lines that serve as internal wavelength standards for calibration of the spectrometers, as shown in Figure 2-4. The lines are due to emission from atomic Xe, and the frequencies of these lines are fundamental, invariant properties of the atoms. The advantage of the frequency calibration is two-fold. First, the mapping of wavelength to pixel is not identical for the reference and sample spectrometers. The intensity spectra must be harmonized so that the intensities at identical wavelengths are used to calculate the absorbance spectrum. Second, the fundamental nature of the Xe emission lines means that the spectrometers in different spectrophotometer units are being calibrated to the same quantity. Therefore, the corrected spectra obtained from different units should be very similar (given similar corrections to the intensities). This property leads to instrument interchangeability, simple calibration transfer, and other advantages described in Section 2.0. 


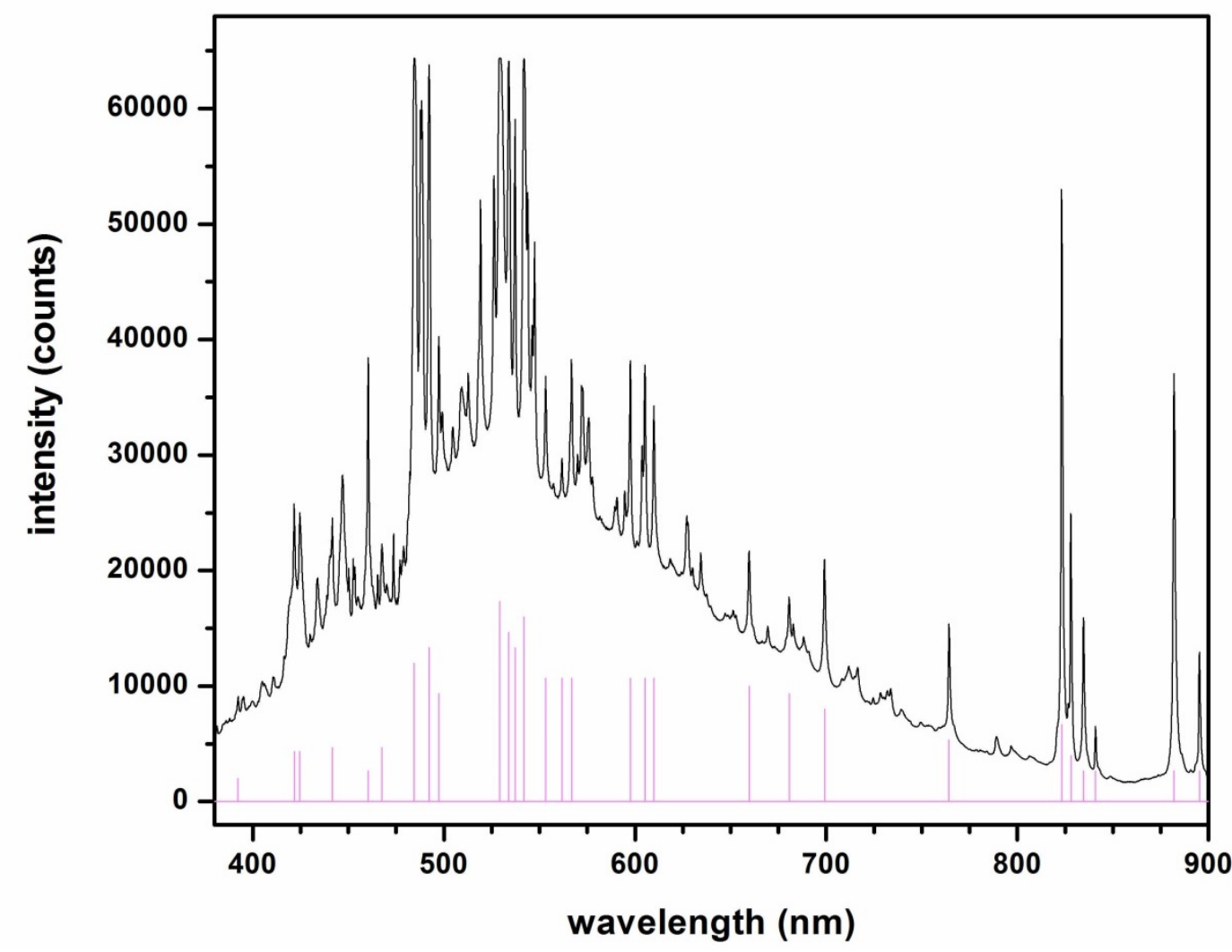

Figure 2-4. Output of xenon flash lamp (top) and expected position of selected xenon atomic emission lines (bottom).

Stable lamp positioning is obtained by having no moving parts between the beamsplitter, irises, light tubes, lamps, and fiber connectors, and by having physical guides to encourage the lamp housings to be placed in the proper place. The light in the reference arm is attenuated with an iris so that the overall intensity is consistent with the light passing through the flow cell and returning to the sample spectrometer. This balancing allows the same integration times to be used for both instruments. Even with the balancing, because the sample path is longer than the reference path and passes through the flow cell where the reference does not, there will be an absorbance offset which must be subtracted from any spectra. This offset is called a blank spectrum. Recording the blank spectrum is functionally equivalent to setting the zero for the colorimeter and is performed under the same conditions (non Pu-containing solution in the flow cell).

To extend the lifetime of the lamps, the spectrophotometers may be turned off if it is known that they will not be used for a week or more. More frequent power cycling tends to degrade the lamps and shorten their lifetime. The spectrometers are expected to return to their previous state upon startup (and after warmup). It is a possibility, but not a certainty, that new blanks and absorbance checks would be required. If there have been changes to the flow cell or fibers, then new blanks and absorbance checks are required.

Spectrometer properties. Miniaturized diode array spectrometers are used in the instrument. These spectrometers operate by allowing the light to be dispersed by a grating and projected onto a linear diode array detector. Each element in the array ("pixel") responds to light intensity over a very narrow range of wavelengths. The output of the spectrometer is thus a spectrum of intensities sampled at a discrete number of points. The device is rugged, with no moving parts, but may be subject to small irregularities that can limit the accuracy of the spectra it obtains. For example, thermal expansion can alter the wavelength calibration. Microscopic irregularities in the grating surface can lead to light scattering 
("stray light") within the device, so that a small fraction of the light of a particular wavelength will be incident on all pixels. Another property of gratings (even ideal ones) allows for a spatial overlap of light with multiples ("orders") of the same frequency (for example, the pixel receiving $800 \mathrm{~nm}$ light will also receive a small fraction of $400 \mathrm{~nm}$ light). Electronic effects can lead to detector noise (at low signal levels) or nonlinear response (at high signal levels). The best quality spectrometers will minimize these effects, but as will be demonstrated, further correction is required to achieve the desired measurement accuracy for this application.

The correction of these effects is not merely desirable from the standpoint of optimizing performance. It is critical for the instrument to work properly. Calculating the solution absorbance requires comparison of the intensities of the same wavelengths from the two spectrometers. While the spectrometers have identical designs, their wavelength-to-pixel calibrations will not be identical. Therefore, there must be a way to convert the calibrations to a common basis for comparison. Similarly, corrections must be made to assure that the detector response to intensity changes is proportionally identical for both spectrometers. This assures that changes intrinsic to the spectrophotometer, such as intensity fluctuations from the light sources, are properly ratioed between the two spectrometers, and that all remaining intensity changes are confidently attributable to changes in solution absorption.

As stated in the discussion of the light sources, real-time wavelength calibration is available from atomic emission lines from the xenon flash lamps, which have well-characterized and invariant wavelengths. [8] The emission lines are also very narrow, less than $0.01 \mathrm{~nm}$, which enhances measurement precision. ${ }^{\mathrm{a}}{ }^{\text {The }}$ spectrometer must have enough wavelength resolution so that individual lines can be distinguished, and are not blurred together. It also must have enough pixels that, despite the narrowness of the lines, each peak is sampled by multiple points. Minimum requirements for spectrometers, and the actual specifications for the devices used in the spectrophotometers, are given in Table 2-1. Note that although the spectral resolution is larger than the intrinsic xenon peak width, it is less than the separation of most xenon lines (see Figure 2-4). Thus, there are many resolvable xenon peaks which can be used for wavelength calibration.

Table 2-1. Spectrometer specifications.

\begin{tabular}{|c|c|c|}
\hline Property & Minimum & Actual $^{(\mathbf{a})}$ \\
\hline Spectral resolution (nm) & 0.4 & $0.30-0.36$ \\
\hline Wavelength range (nm) ${ }^{(\mathrm{b})}$ & $375-875$ & $356-916$ \\
\hline Pixel count & 2500 & 3648 \\
\hline Pixel spacing (nm/pixel) & 0.2 & 0.154 \\
\hline Stray light (at 520 nm) & $<0.05 \%$ & $0.04-0.1 \%$ \\
\hline Sampling speed (ms/scan) & $<5$ & 3.7 \\
\hline Resolution, Analog/digital converter & $16-$ bit & $16-$ bit \\
\hline
\end{tabular}

(a) Actual = Avantes AvaSpec-ULS3648: $600 \mathrm{~mm}^{-1}$ grating, $10 \mu \mathrm{m}$ entrance slit, order-sorting grating (350/600 nm long-pass filter).

(b) Selected to cover Pu absorbance peaks.

The procedure for real-time wavelength calibration includes real-time detection of the xenon peaks, comparison of the measured and expected wavelengths, and application of any corrections to the wavelength calibration. This procedure is done for every spectrum. Figure 2-5 shows an expanded portion of the intensity spectrum. For each discrete point in the spectrum (except the two leading and two trailing pixels), the measured intensities are binned into a 5-pixel window (shown in red). The intensities

\footnotetext{
${ }^{a}$ Also note that the xenon peaks are much narrower than the Pu absorbance peaks, which are $\sim 20-50$ nm wide. Therefore, the shape and position of the xenon peaks measured with the sample detector will not be altered by absorbances in the flow cell.
} 
at these five points are fit to a quadratic equation, $I(\mathrm{x})=a_{0}+a_{1} \mathrm{x}-a_{2} \mathrm{x}^{2}$, where $\mathrm{x}$ is the position relative to the center of the window. In this formulation, $a_{2}$ will be large and positive if there is a xenon peak present within the window. Thus, if the fit value of $a_{2}$ is below a threshold value, it is assumed that no peak is present, and the window is moved over one pixel and the evaluation process is restarted. If a peak is indicated, the center of the peak is estimated as $a_{1} / a_{2}$, corresponding to the condition of the first derivative being equal to zero. In this way a table of peak positions is formed. The rough wavelength values for the peaks can be estimated from the manufacturer's factory calibration. This allows the peaks to be matched to reference values. The table is refined to include $\sim 30$ xenon lines. A third order polynomial is then fit to provide a single correction function that is applied to the wavelength calibration.

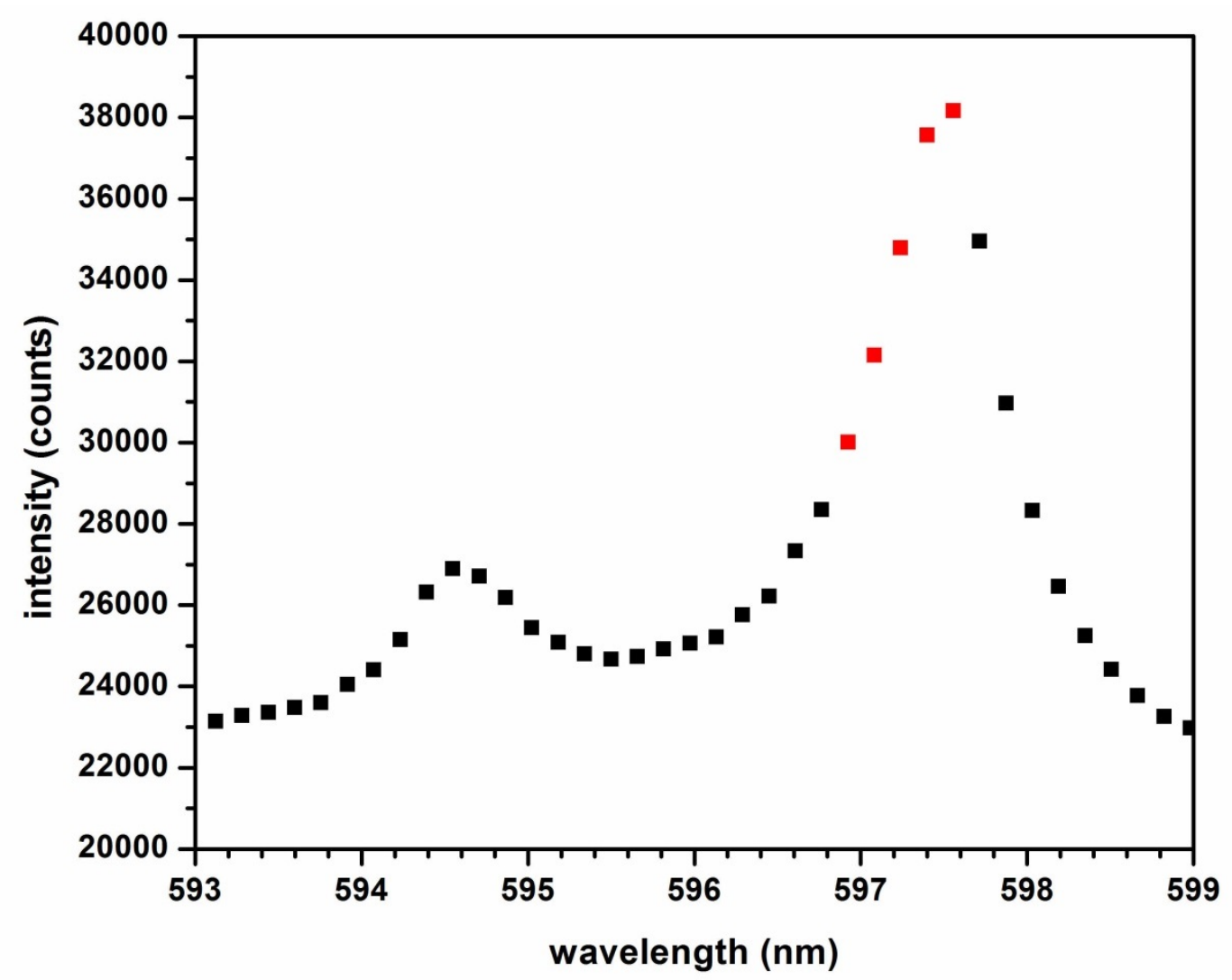

Figure 2-5. Expanded portion of Xe lamp emission, showing multiple pixel points per emission line.

This process is typically able to match xenon peak positions with a root mean square (RMS) error of 0.02 $\mathrm{nm}$, or approximately $1 / 8^{\text {th }}$ of the spacing between pixels. This compares favorably with the accuracy of the factory calibration, which can be as high as $0.5 \mathrm{~nm}$ RMS error. The significance of this accuracy improvement can be estimated by observing the effect on Pu measurement associated with wavelength error. A Pu calibration model (as described in Section 4) was applied to otherwise identical spectra that were shifted by fractions of a nanometer. The changes in the observed reading are shown in Figure 2-6. A shift of $\pm 0.02 \mathrm{~nm}$ from the true wavelength value, indicated by the orange lines, corresponds to a $0.1 \%$ error in predicted value. The blue lines represent the positions of individual pixels. A 1-pixel error ( $\sim .15$ $\mathrm{nm}$ ) corresponds to a Pu measurement error of $-2 \%$, and errors as large as $\pm 0.3 \mathrm{~nm}$ (almost 2 pixels) correspond to a $6 \% \mathrm{Pu}$ measurement error. Thus, rigorous wavelength calibration is critical for accurate Pu measurements. 


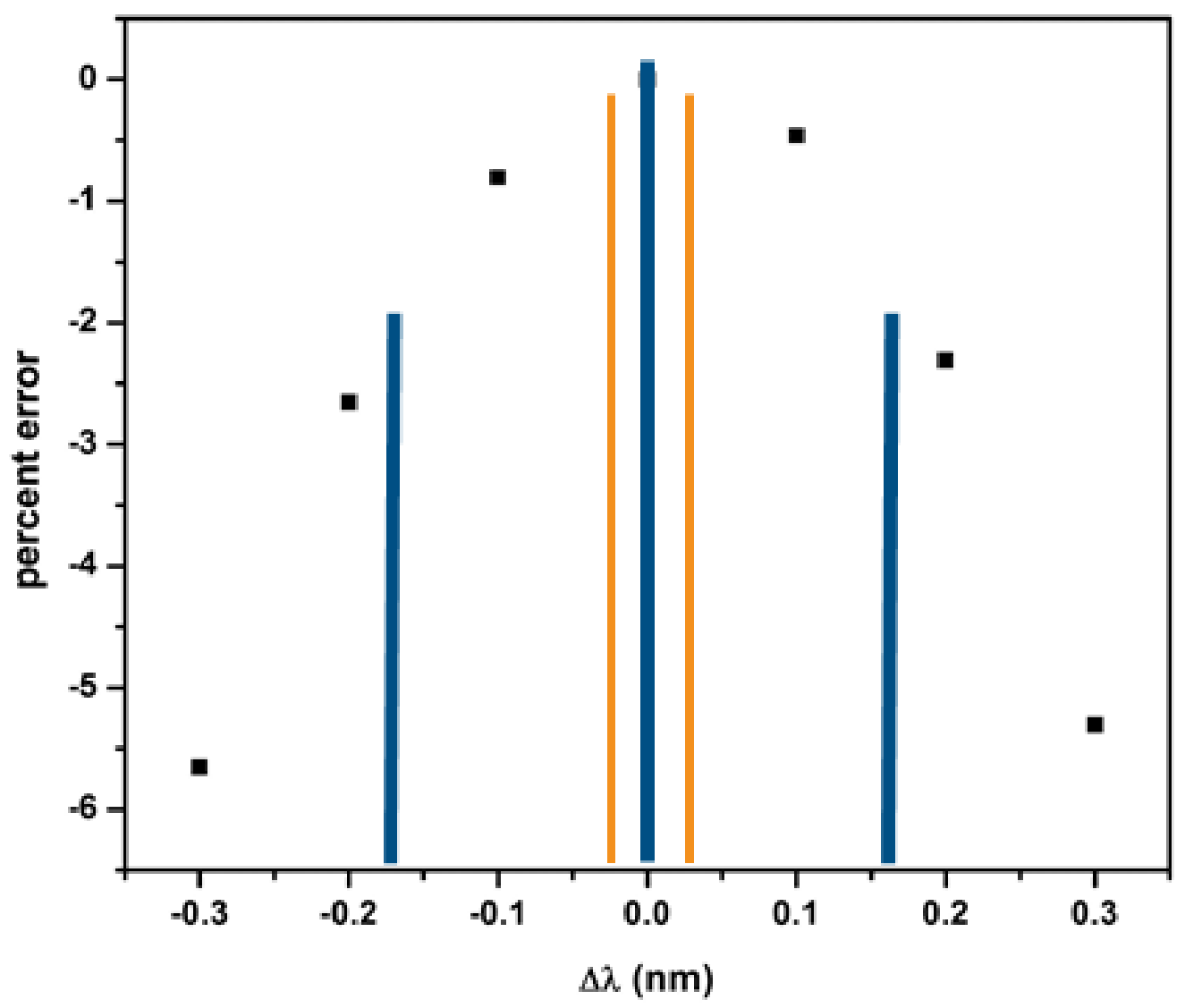

Figure 2-6. Effect of wavelength calibration error $(\Delta \lambda)$ on Pu prediction.

Intensity calibrations assure that proportional changes to light intensity are measured in all parts of the instrument response range. The dominant sources of response nonlinearities arise at the low intensity/high absorbance end from dark current and stray light. At the high intensity/low absorbance end, nonlinearities from charge readout error can occur. Dark current is a background electrical signal due to thermal excitation of electrons in the circuit. [9] It provides an offset signal that can be comparable to low incident light levels. In the spectrometer, there are several pixels at the end of the array that are physically masked so that they are not exposed to light. These pixels are read to estimate the dark current, and the average is subtracted from every spectrum as a dynamic dark correction. Figure 2-7 shows the residual signal level (grey dots) after dark subtraction, which is noise with a peak-to-peak height of $\sim 15-20$ counts. This value indicates the lowest light levels that can be measured reliably, even if all other noise sources are eliminated. 


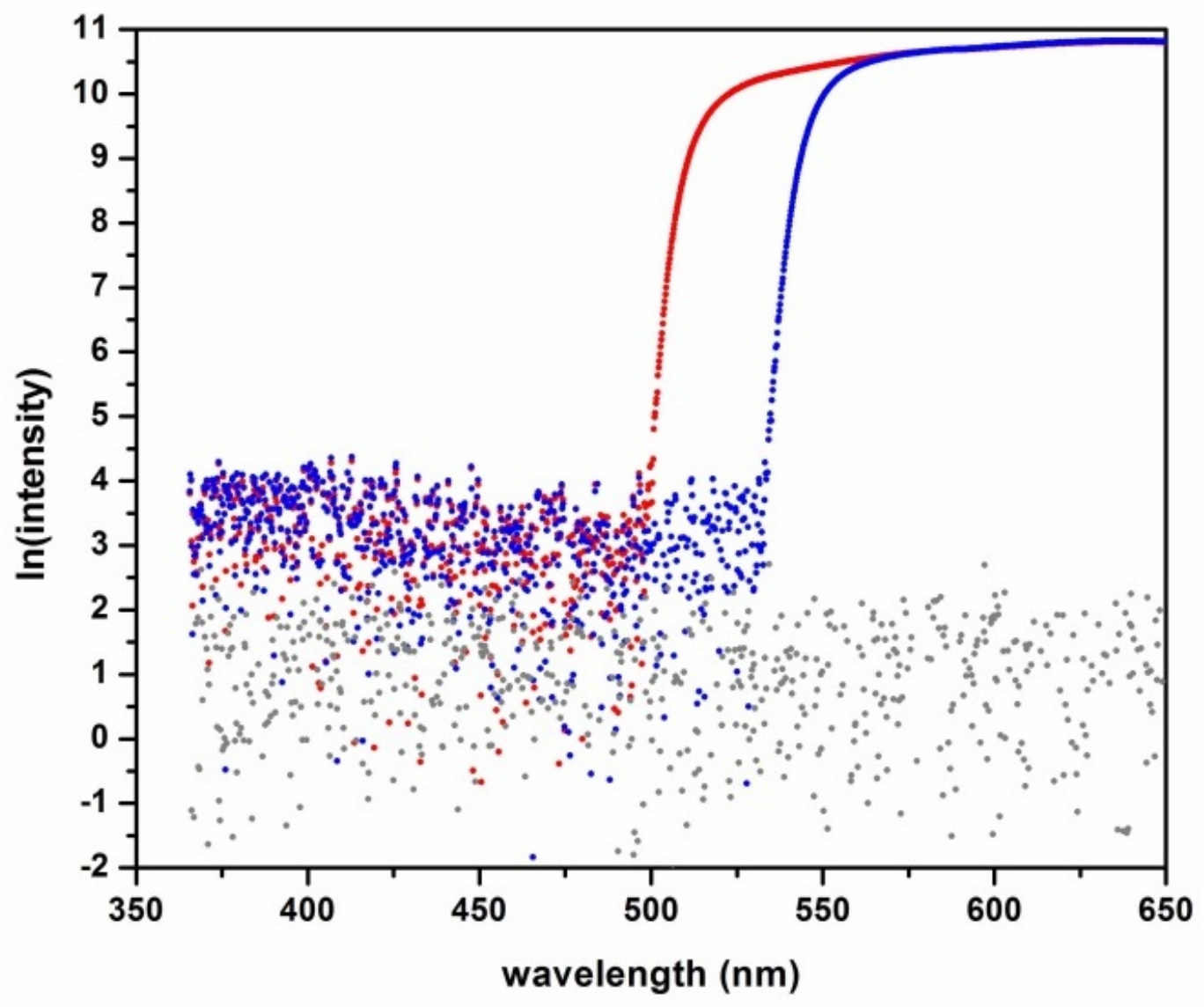

Figure 2-7. Corrected dark current (grey) and observed stray light levels (red/blue).

Stray light is light of any wavelength which strikes the detector at a location other than the pixel associated with its wavelength. It occurs mainly due to scattering from microscopic irregularities of the optical components (grating, mirrors) of the spectrometer. This effect occurs at every pixel but is more prevalent at shorter wavelengths. [10] Figure 2-7 shows the measurement of stray light in a spectrometer. A long-pass filter (red curve: $500 \mathrm{~nm}$ cut-off; blue curve: $550 \mathrm{~nm}$ cut-off) prevents light at wavelengths below the cut-off from entering the spectrometer. Any light observed at the shorter wavelengths is therefore stray light of a longer wavelength. This light level is on the order of $0.05 \%$ of the light intensity at longer wavelengths (20 counts out of 40,000). The amount of stray light will be essentially invariant with time and changes in environmental conditions. Therefore, a one-time corrective formula, determined during instrument development, will suffice for future spectra. This formula is applied during data acquisition.

Order overlap is separate effect which preferentially appears at longer wavelengths. This phenomenon is a property of gratings, in which harmonics of a given wavelength will be coincident on the same detector pixel. To counter this, well-designed spectrometers will have internal "order-sorting" filters mounted in front of the pixel array. [11] These filters are part of the specifications for the spectrometers used in these instruments. However, it is prudent to perform checks to assure that these filters are in place. This is done by using short pass filters, which block longer wavelengths of light, and observing if the apparent long wavelength intensity increases with the presence of short wavelengths of light. If necessary, another corrective formula can be applied in tandem with the stray light formula described above. 
The following example illustrates the significance of these effects. The strongest absorbance peak for $\mathrm{Pu}^{4+}$ is at $\sim 475 \mathrm{~nm}$. The intensity of the combined sources at this wavelength will be approximately 20,000 counts, with higher intensity at longer wavelengths. A solution absorbance of 2.2 at $475 \mathrm{~nm}$ should result in $\sim 125$ counts reaching the detector. However, an uncorrected stray light level at $475 \mathrm{~nm}$ equal to $0.05 \%$ of the longer wavelength light would add $\sim 20$ counts to the measurement, resulting in 145 counts at the detector. Therefore, the measured absorbance would be $2.14\left(\log _{10}(20,000 / 145)\right)$, which is a bias of about $-3 \%$ from the true value. This potential bias is eliminated with the corrections described above. The limiting factor for accurate measurement at high absorbance is therefore the random (thermal) detector noise, which provides a 1-sigma random uncertainty of about \pm 3 counts (roughly 1/3 the peakto-peak noise of \pm 9 counts). For the present example, this corresponds to an absorbance uncertainty of $0.95 \%$ (range of $\mathrm{A}=2.194-2.215$ ). This results suggests that $\mathrm{A}=2.2$ is a suitable upper limit of absorbance for including a data point in the subsequent determination of Pu concentration. Section 3 will describe how the absorbance spectra are evaluated for such high-absorbance points, and how different $\mathrm{Pu}$ calibrations may be selected based on such an evaluation.

Absorbance standards and check filters. Two types of external standards will be used to certify the absorbance measurements of the spectrophotometers. Both types have characteristic absorption spectra, which are shown in Figure 2-8. Metal-oxide neutral density (ND) filters are grey filters that have a comparatively flat response across the visible spectrum. The absorbance values at specific wavelengths are traceable to the National Institute of Standards and Technology (NIST) [12] and can be recertified by vendors who maintain ISO-certified programs. ${ }^{a}$ Holmium (Ho) oxide filters have characteristic peaks and are typically used as wavelength standards. In this application, the filters will be used both as a check

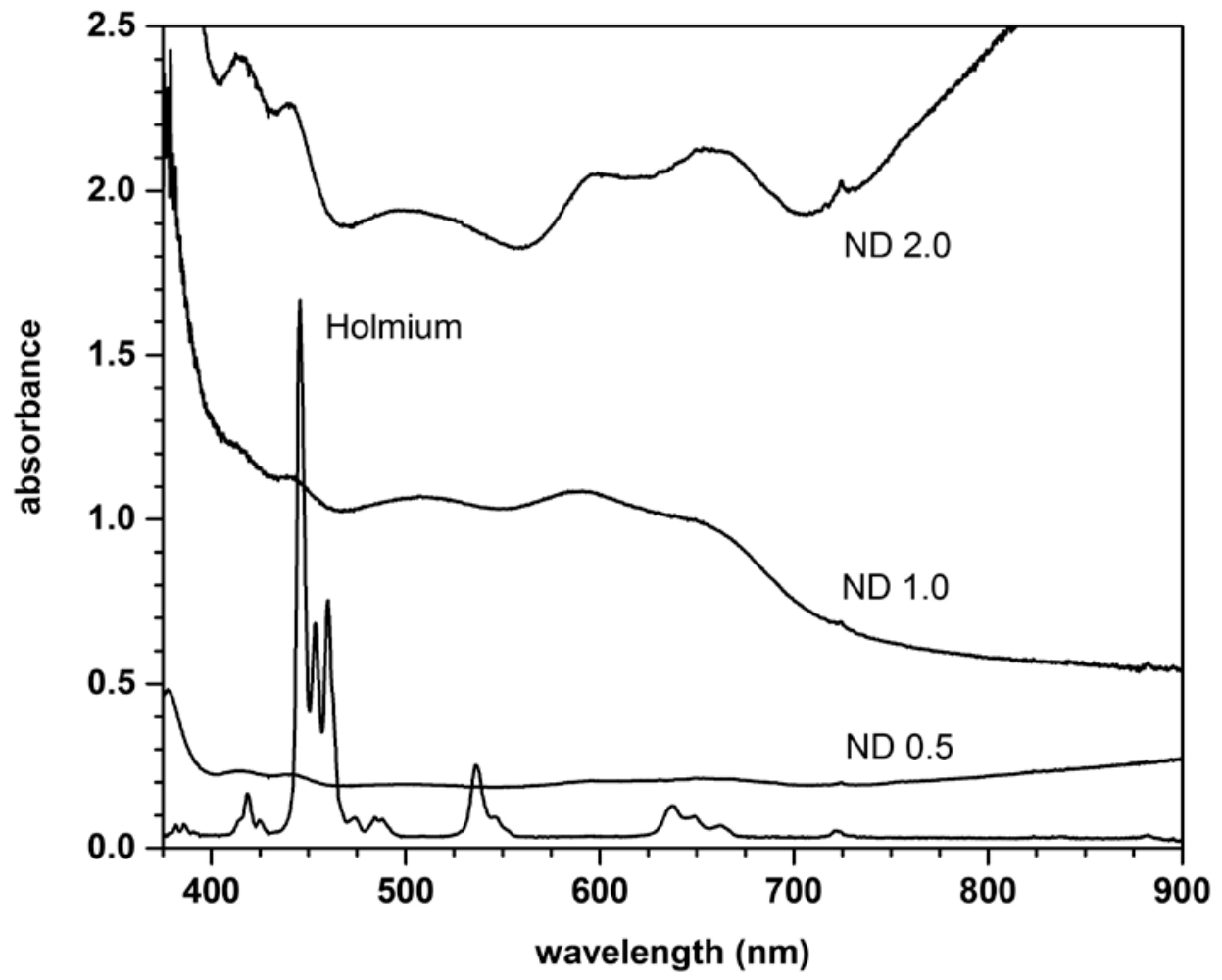

Figure 2-8. Example absorbance spectra of spectrometer calibration standards.

\footnotetext{
${ }^{\mathrm{a}}$ For example, the vendor FireflySci (Brooklyn, NY) is certified to ISO-9001 and ISO-17025.
} 
filter to provide an on-demand performance check and as a nontraceable absorbance measurement check. As a check filter, the Ho oxide filter will generate an instrument response when inserted into the light path. The consistency of the response with the expected value, obtained when the instrument was initially calibrated, indicates that the instrument continues to work properly. The absorbance values for several peaks in the Ho oxide spectrum will also be used as an informal check of absorbance response. The check is informal, because Ho oxide filters are not certified for absorbance, but the peaks span a larger absorbance range than is available for the neutral density filters, and the information obtained thereby is valuable.

The recommended renewal rate for the neutral density and Ho oxide filters is one year. Note that there is low risk if circumstances require an extension of the calibrations. Both filters are comprised of a highly mechanically and chemically stable metal oxide film with a protective quartz coating. The NIST recommendation for recertification is every two years. As long as the standards are stored and handled properly (e.g. protected from scratches and fingerprints, stored in the case, etc.), the standards should give consistent values for an even longer period of time. NIST has asserted that holmium oxide glass wavelength standards "have shown insignificant spectral variation from batch to batch and from one manufacturer to another", that over four decades "there has been no recorded instance of a spectral shift of the certified bands for any of the samples measured", and that the samples "are known to be robust and relatively insensitive to a normal range of temperature and humidity". [13] It is further stated that "holmium oxide is inherently more stable than most instruments [used to measure the absorption bands]" and can be considered as an intrinsic standard. The standards vendor claims that a study of their product by the National Research Council of Canada has demonstrated no shift in absorbance values for at least 8 years.

The check filter is inserted in the sample arm of the spectrometer. After passing through the filter, the light in that arm goes through the flow cell and return fiber before entering the sample spectrometer. Therefore, checks should be done with a recently flushed flow cell (and new blank if required) and with fibers in a stable position. A rule of thumb for flushing the flow cell is that the solution should remain clean for at least one shift after flushing. Over longer periods of time, Pu may leach from the piping. Also, a flow cell that is allowed to go dry may have deposits left on the window which must be removed by flushing. A spectrometer reading above $0.05 \mathrm{~g} / \mathrm{L}$ or qualitative evidence of Pu from observation of the absorbance spectrum are indications that a new flush is required.

Both the check filter mechanism and cuvette holder were chosen so that the standards in each would intersect the light path consistently. The cuvette holder has a spring-loaded set screw in its base which pushes the cuvette (a square-sided sample holder) flush against a flat, fixed surface. The check filter is mounted so that the axis of its rotation is parallel to the sample light path, and the filter itself is always perpendicular to the beam. Thus, the check filter reading should not depend on minor variations in the positioning of the check filter arm, as long as the entire beam passes through the filter. Enforcement of this configuration is a lesson learned from the raffinate colorimeter, where the rotation axis was perpendicular, and small misalignments led to inconsistent readings as the filter was titled into nonnormal orientation to the beam.

Optical fibers and flow cells. The existing fibers and flow cells are compatible with the spectrophotometers and will be reused. In the interest of maintaining information about all spectrophotometer components in a single document, their properties are described below. The appendices include a parts list and instructions for construction and testing for both.

Fibers may be obtained from several vendors, but have these common characteristics. [14] They are UVenhanced multimode fibers, with a 400 micron high-OH core, 440 micron doped silica cladding, Kevlar strength members, and an outer polyimide jacket. Some fibers are packaged in a duplex configuration, 
with two of the above fibers in parallel inside a larger polyimide jacket with additional Kevlar fibers. The numerical aperture of the fibers is 0.22 . The fibers are terminated with nickel-free SMA 905 connectors and rubber sleeves for strain relief. Fibers are held in place in the connectors with epoxy, and are polished and inspected at SRNL. Fibers which are inside the production glove boxes are removed from the outer jacket and inserted into a flexible stainless steel jacket. The fiber is sealed at the ends of the steel jacket with epoxy and shrink wrap tubing. Detailed work instructions for construction and testing of the optical fibers are given in Appendix I.

The flow cells are constructed from $1 / 2$ " stainless steel unions. The optical region is defined by two collimating lenses present at the opposite horizontal ends of the union. An expanded view of the lenses is provided in Figure 2-9. The lenses have 316L stainless steel lens housings which accept compression fittings for installation in the union. The primary internal seal is provided by compression of a Kalrez oring against an internal shoulder of the lens housing by the lens retainer, which is inserted by means of screw threads. The quartz lens that collimates the light from the optical fiber is epoxied to the lens retainer. The outer end of the collimating lens presses against the internal shoulder of the union. Together the two lenses define a 1" path length. (Shorter lengths may be obtained by drilling out these internal shoulders, although that is not done for this application.) Optical fibers are coupled to the lenses with a SMA 905 bushing. The relative position of the SMA fiber to the collimating lens is established with a stainless steel locking nut. Part of the flow cell construction procedure involves assuring that the face of the fiber is at the focal point of the lens. In this position, the light is most efficiently collimated and the throughput is maximized. Finally, strain protection is provided by an extension tube. Detailed work instructions for construction and testing of the flow cell are given in Appendix $\mathrm{H}$.

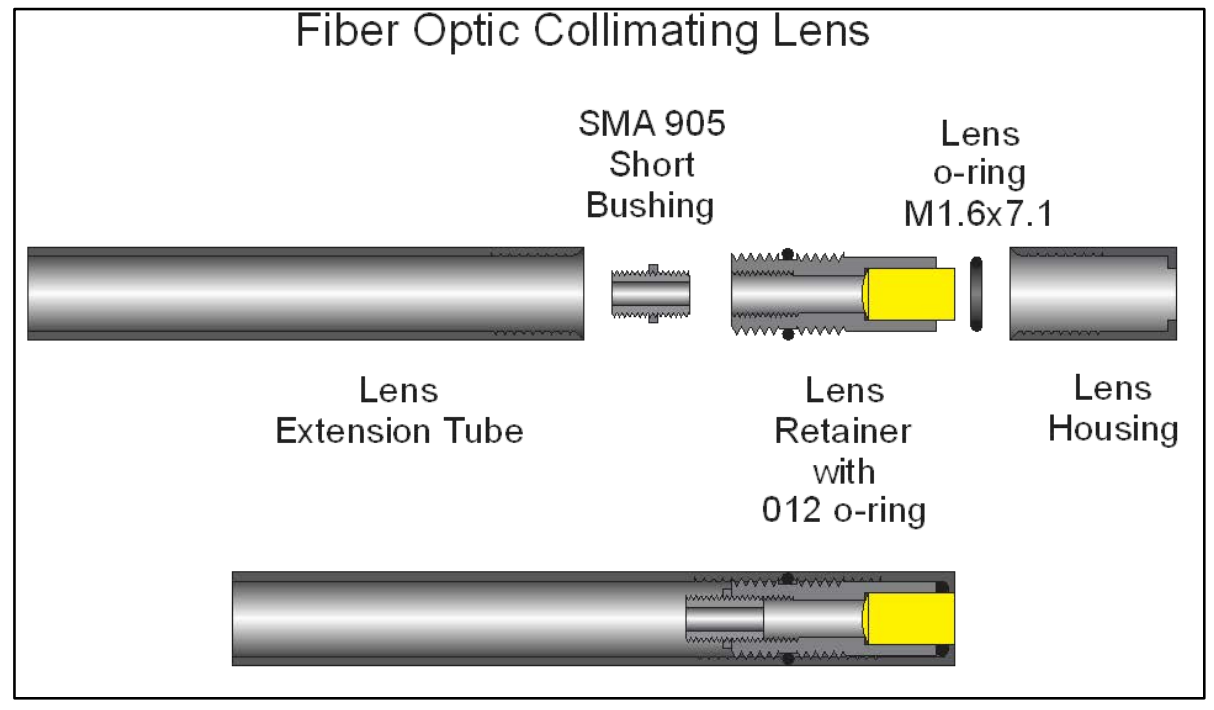

Figure 2-9. Schematic diagram of collimating lens.

\subsection{Relationship to facility and common mode analysis}

Figure 2-10 depicts the relationship between the different top-level components of the instruments, the display hardware, and the facility power supply and the DCS. Connections are color-coded by type, e.g. power, USB/ethernet, and 4/20 mA signal, with arrows depicting the direction of communication. The Uninterrupted Power Supply (UPS) is connected to a $120 \mathrm{~V}$ facility power supply. The UPS in turn powers the computer, spectrophotometer, and I/O modules for each instrument, as well as the display monitor and switch. The heavier dotted line denotes the boundary of the Component Location Identifier (CLI) for the entire spectrophotometer system. The lighter dotted lines denote potential CLI groupings 
for individual spectrophotometers, although these CLIs have not been formally declared as of the time of this report.

From a cyber security standpoint, interaction with the facility only occurs through the DCS. Specifically, the connections are 4-20 mA (Pu concentration) and 0-24 V (alarm) from each spectrophotometer I/O module to the DCS. There is no communication in the opposite direction (facility to instrument). The spectrophotometer computers are isolated from any outside network, including any Site or facility network.

The common point of interaction for the three spectrometers is the uninterrupted power supply / power conditioner. Testing at SRNL showed that when running simultaneously, the three spectrophotometers and associated equipment used $190 \mathrm{~W}$ of power, representing a load at $14 \%$ of the capacity of the UPS. When the UPS was removed from building power, the instruments continued working without interruption. The battery was reduced to $10 \%$ after 46 minutes. After repowering the UPS, the battery charged at a rate of approximately 3\% every 5 minutes. The power conditioner is intended to reduce the effect of electrical noise on the instrument output. If noise does reach the instruments, the primary effect should be random noise on each spectrometer, which would increase the uncertainty of the reading. It is untested if such noise would result in a bias in the instrument reading, or if the instruments would change in a common direction. Note that because the lamps are split evenly between the reference and measure spectrometers within each instrument, fluctuations in lamp intensity or wavelength output will affect both channels equally and have minimal effect on the absorbance spectrum, as shown in Figure 2-3.

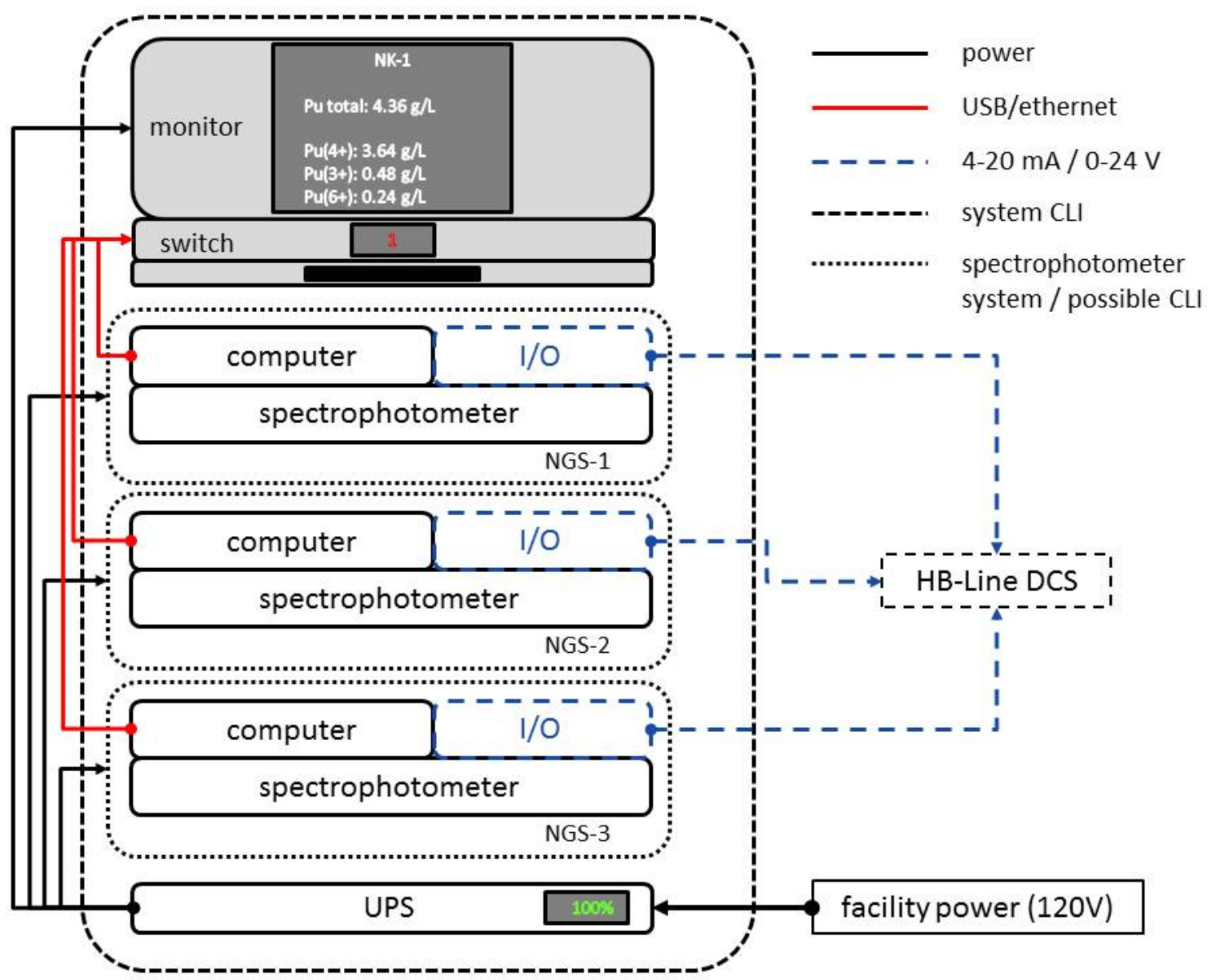

Figure 2-10. Power and communication connections for the spectrophotometers. 


\subsection{Control Software}

\subsection{Overview.}

A new instrument control software program has been written in order to take full advantage of the developments in hardware and data analysis associated with the spectrophotometers. The program is called NGS-Main (version 1.0) ${ }^{\mathrm{a}}$ and is activated by double-clicking the labeled icon on the computer screen. The program performs the following functions:

- Data acquisition and processing, including control of the spectrometers and lamps, application of wavelengths and intensity calibrations, and processing the data to the "virtual instrument" basis, as well as evaluating diagnostics for system health and data quality;

- Data interpretation, i.e. analysis of the absorbance spectrum to extract $\left[\mathrm{Pu}_{\text {total }}\right]$ and the distribution of Pu oxidation states;

- Communication of reading results and instrument status to the "outside", both on-instrument display and DCS;

- Archiving of raw and processed spectra and analysis results; and

- Automated and/or guided calibration and verification using wavelength standards.

Work instructions for performing calibration checks are given in Appendix B. Instructions for measuring / resetting the blank spectrum are given in Appendix C.

\subsection{Programs and tools.}

The SRNL instrument control program is written in the Visual Basic environment (VB.net, version 4.0). Individual instrument components, where required, are controlled through instrument vendor drivers. These include:

- Avantes spectrophotometer: AS5216.dll (version 2.2.0.0) and AvaSpec-USB2.dll (version 1.6.0.1), installed using setupAvaSoft7USB2.exe (version 10.3.0.250).

- Opto-22 I/O modules: OptoMMP3.dll (version 3.0.0.0).

- ActiveX interpreter for ThorLabs check filter mechanism: AxInterop.MG17SystemLib.dll (version 1.0.0.0) and AxInterop.MG17MotorLib.dll (version 1.0.0.0).

- Eigenvector analysis model interpreter: ModelExporterInterpreter.dll (version 1.0.0.0).

These programs are run in the Windows 7 environment.

\subsection{Quality assurance.}

The NGS HB-Line software has been identified and documented in Software Classification Document (B-SWCD-H-00611) as General Safety (GS) per the requirements of Manual E7, Procedure 5.01. Per the SWCD a graded approach to Software Quality Assurance (SQA) will be applied to increase the reliability of the spectrophotometer units to that of a Safety Significant (SS) component based on the units' association with criticality administrative controls. The Software Quality Assurance Plan implementing this approach is defined in document B-SQP-A-00070, Next Generation Spectrophotometer (NGS) - HBLine Software Quality Assurance Plan (SQAP).

\footnotetext{
${ }^{\mathrm{a}}$ NGS $=$ Next Generation Spectrometer.
} 


\subsection{Program flow.}

A top-level schematic diagram of the program is shown in Figure 3-1, which lists the inputs and outputs between the modules. On startup, the configuration and model files are read once into the spectrometer and model subsystems, respectively, and are stored in memory for the duration of the time that the program is running. The spectrometer subsystem immediately starts to acquire data according to parameters found in the appropriate configuration file. Once the reference and sample intensity spectra are obtained, they are corrected, converted to a standard wavelength basis, and transformed into an absorbance spectrum. The spectrum and status flags arising from diagnostic checks are passed to the main module, which is waiting for them passively. The spectrometer subsystem also writes the raw intensity spectra, corrected spectra, and status to archive files. After the spectrum and status signals have been passed to the main module, the spectrometer subsystem starts to take the next set of spectra. The only signal which goes from the main module to the spectrometer subsystem is an on-demand signal to insert the check filter, which is activated by the toggle switch on the front of the display panel.

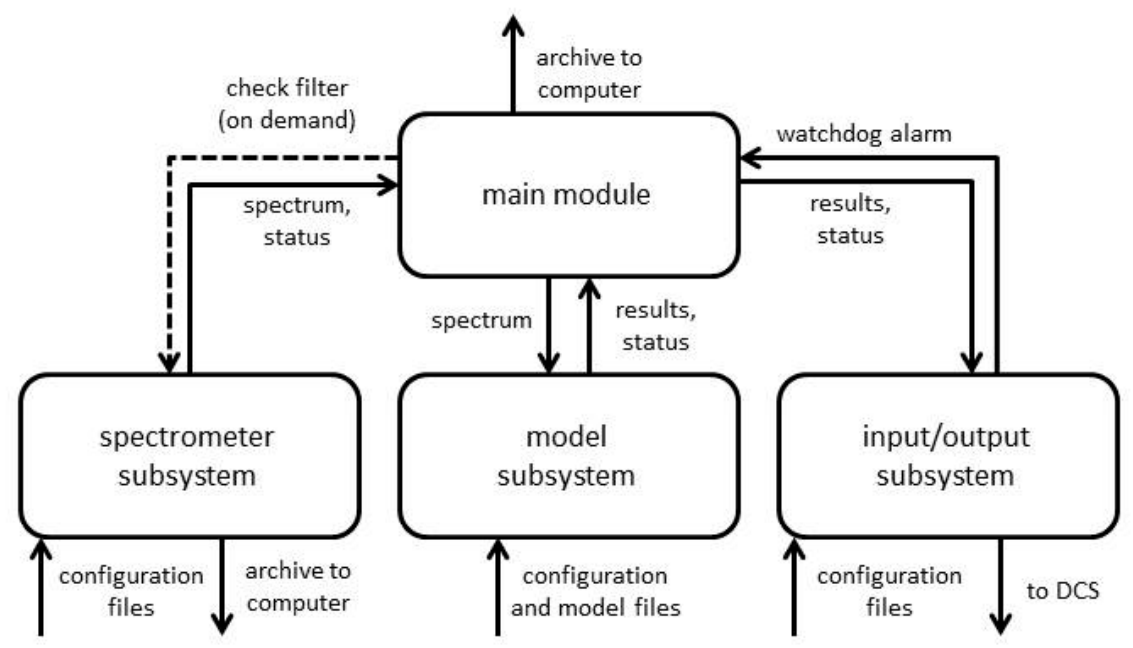

Figure 3-1. Top-level schematic for control software operation.

The main module passes the converted spectra to the model subsystem, which interprets the data to determine the total Pu concentration and the concentrations of the individual Pu oxidation states. These results are returned to the main module. The module then passes the results to the input/output (I/O) subsystem, which converts the total Pu concentration to an analog (4-20 mA) signal and any status alarms to a digital signal and passes those to the DCS. The main module also presents the results from the model subsystem to the instrument display. The I/O system autonomously maintains a watchdog alarm to assure that the main module is running properly. All of these steps occur in parallel with the data acquisition by the spectrophotometer subsystem.

The modular organization of the program separates the different functions so that future upgrades to individual components will have minimal impact on the program as a whole. Examples of such changes include the use of a different spectrometer or upgraded drivers, or the use of a different data analysis program. Furthermore, all configuration settings are contained in separate data files which are read in upon program startup. New Pu prediction models, integration times, or settings for diagnostics can be changed without requiring a new version of the underlying program. 


\subsubsection{Spectrophotometer subsystem.}

The operation of the spectrophotometer subsystem is shown in Figure 3-2. Specific details for each step are as follows.

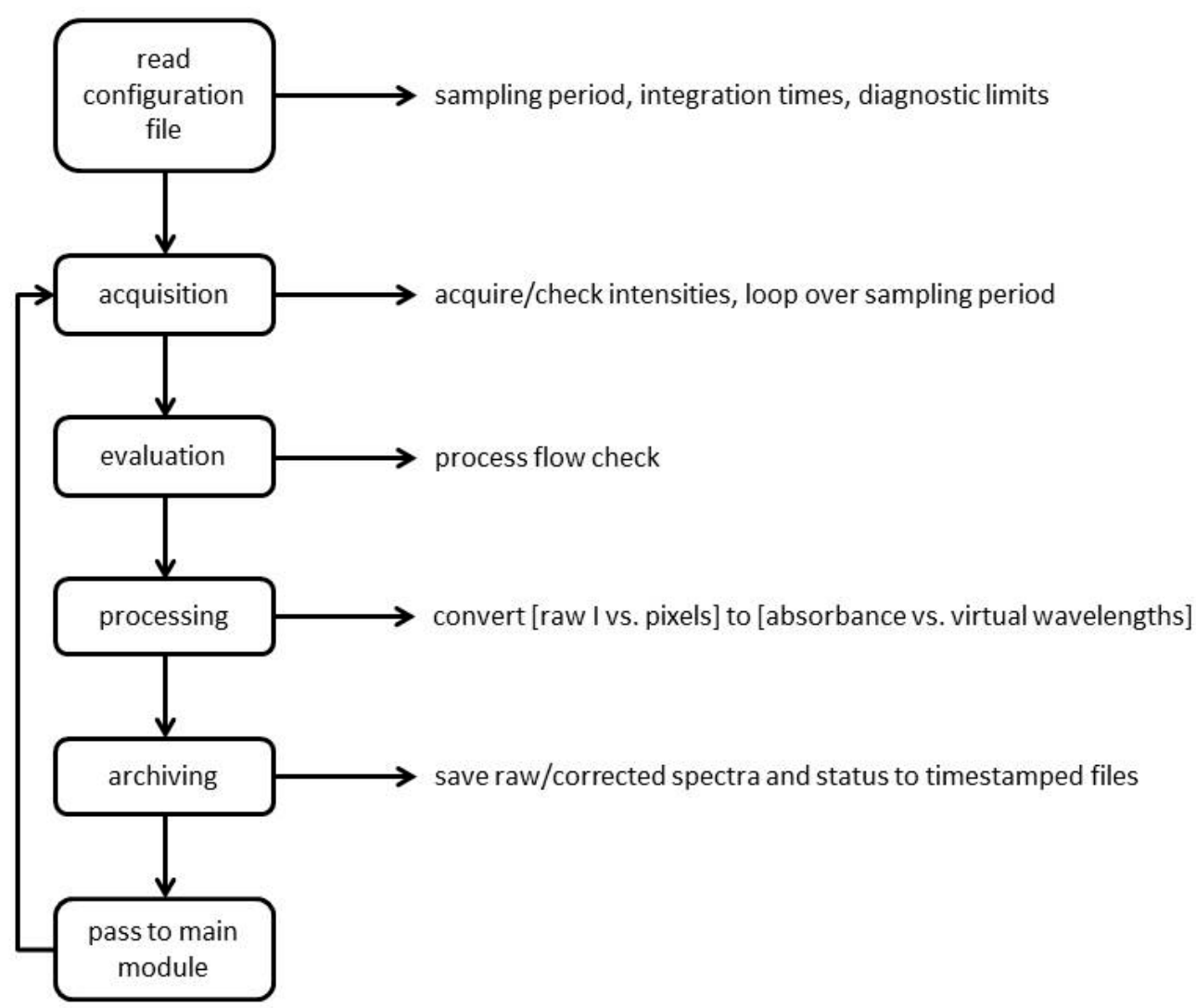

Figure 3-2. Flow diagram for spectrophotometer subsystem.

- $\quad$ Reading configuration files. This is done upon instrument startup, with all parameters stored in memory. Important parameters read during this time include spectrometer identification (as reference and sample), integration times and spectral averages, number of flashes of the xenon lamp per integration period, overall sampling period during which spectra are accumulated, and diagnostic limits. Any changes made to the files during troubleshooting while the program is running are simultaneously written to memory and saved to the configuration file, to be available during the next startup.

- Data acquisition. This section has two nested loops. During the smaller loop, a fixed number of intensity measurements are acquired and averaged on each spectrometer. The intensities are passed to the computer. The reference spectrometer signal is evaluated for evidence of low intensity for either lamp. The reference signal is used for this purpose because the intensity will not be affected by the solution in the flow cell. The signal on the sample detector is evaluated to see if there is adequate transmission through the flow cell (a minimum intensity is expected at $\lambda$ $580 \mathrm{~nm}$, which is the wavelength of minimum Pu absorbance). Failure of any of these tests will trigger a status warning and the data from both spectrometers will be discarded. If the tests are good, all the data is accepted. Working with the data as matched sets assures that instantaneous lamp variations that equally affect the reference and sample channels will be tracked and properly compensated. The larger loop repeats the smaller loop as many times as possible within the overall sampling period. Typically, there will be 5 measurements averaged within the smaller 
loop, and the overall sampling period will be 5 seconds. The total number of intensity measurements that can be obtained within the 5 second period will depend on integration times and some overhead time associated with data transfer; in laboratory tests, up to 68 spectra could be obtained in the 5 second period.

- Data evaluation. Once the overall sampling period is completed, there will be several matched sets of intensity spectra for each detector. The standard deviations for each set are compared. If the variance of the data from the sample arm exceeds that of the reference arm by more than a certain amount, there is turbulence in the flow cell that can compromise the data. The status flag for "Confidence" is activated.

- Data processing. Here, the data are transformed from an intensity versus pixel basis to an absorbance versus virtual wavelength basis. The first step is to correct the intensity spectrum on each detector separately for stray light, second-order overlap, and detector readout error. The corrections for these effects have been measured relative to the pixels on each detector. Next, the pixel positions are converted to wavelengths, using the positions of the xenon emission lines as an internal standard. ${ }^{\mathrm{a}}$ The spectra are then interpolated to a standardized basis set of wavelengths ( $200-899.8 \mathrm{~nm}$ with $0.2 \mathrm{~nm}$ spacing, for a total of 4500 points). At this point, the reference and sample intensity spectra are directly comparable. The absorbance for each interpolated wavelength is calculated from the Beer-Lambert Law. The final absorbance spectrum is then calculated by subtracting the blank absorbance spectrum, which represents the apparent absorbance of the sample channel relative to the reference channel arising due to differences in the fiber lengths and the presence of the flow cell in the sample path. Acquisition of the blank spectrum is equivalent to "zeroing" the instrument. When the blank spectrum is acquired, it is simultaneously read into memory and stored on disk in a time-stamped file. All blank spectra are saved, and the most recent blank is read when the program is started.

- Archiving. Data saved to the disk include the raw intensity spectra, the transformed absorbance spectra, and any associated status flags. These spectra are cross-indexed to the sample analysis results generated in the model subsystem.

- Communication. The transformed absorbance spectra and the status flags are passed to the main module for further processing. The subsystem then returns to the acquisition step and starts over.

The execution of the data evaluation and processing, archiving, and communication steps requires only $\sim 0.15$ sec. This is a small period of time compared to the typical sampling period of $\sim 5 \mathrm{sec}$ and does not constitute a significant delay in reporting results.

\subsubsection{Modeling subsystem}

The absorbance spectrum obtained with the spectrometer subsystem is analyzed in the modeling subsystem to determine the concentrations of total plutonium and the distribution of the individual oxidation states. The modeling flowsheet is designed to classify the absorption spectrum into one region within the total range of conditions (concentration, acidity, disproportionation) in which the process solution may fall. Within each region, the models are simpler and more accurate than could be obtained if a single model was used to cover the entire range of conditions.

During instrument startup, a number of files are read into this subsystem. These include the models for acidity classification, $\left[\mathrm{Pu}_{\text {total }}\right],\left[\mathrm{Pu}^{3+}\right]$, and $\left[\mathrm{Pu}^{6+}\right]$. There is also a configuration file which includes information including the pathlength of the flow cell (which is as a multiplicative factor, assuming that the prediction models were based on data obtained through a $1 \mathrm{~cm}$ cuvette), threshold absorbance values, wavelength ranges, and some information about the individual models that is used for monitoring and diagnostic purposes.

\footnotetext{
a Large Pu concentration may lead to the xenon lines being obscured on the sample channel. If this occurs, the previous "good" wavelength calibration is applied to the data. Wavelength drift over the period of time associated with the heart cut is minimal.
} 
The decision tree for classification is shown in Figure 3-3. The first decision applied to the spectrum is to determine which data points may be included in the analysis. The upper limit to solution absorbance is 2.2. The wavelength range that is most likely to have an absorbance above that value is between 460-510 $\mathrm{nm}$, which corresponds to the dominant peak in the Pu nitrate spectrum. [15] If the absorbance is less than 2.2 in that range, the spectrum is classified as "low absorbance". If the absorbance is greater than 2.2, then the 600-700 $\mathrm{nm}$ region is evaluated, corresponding to the next largest $\mathrm{Pu}^{4+}$ peak. Spectra which pass this test are considered "medium absorbance". If necessary, a third region (505-530 and 550-600 nm) is evaluated, with spectra passing the test being considered "high absorbance". Spectra with excessive absorbance in this region are considered "saturated". For this last case, the total Pu concentration is indicated to be greater than the maximum value of the instrument and the oxidation state distribution is left undetermined.

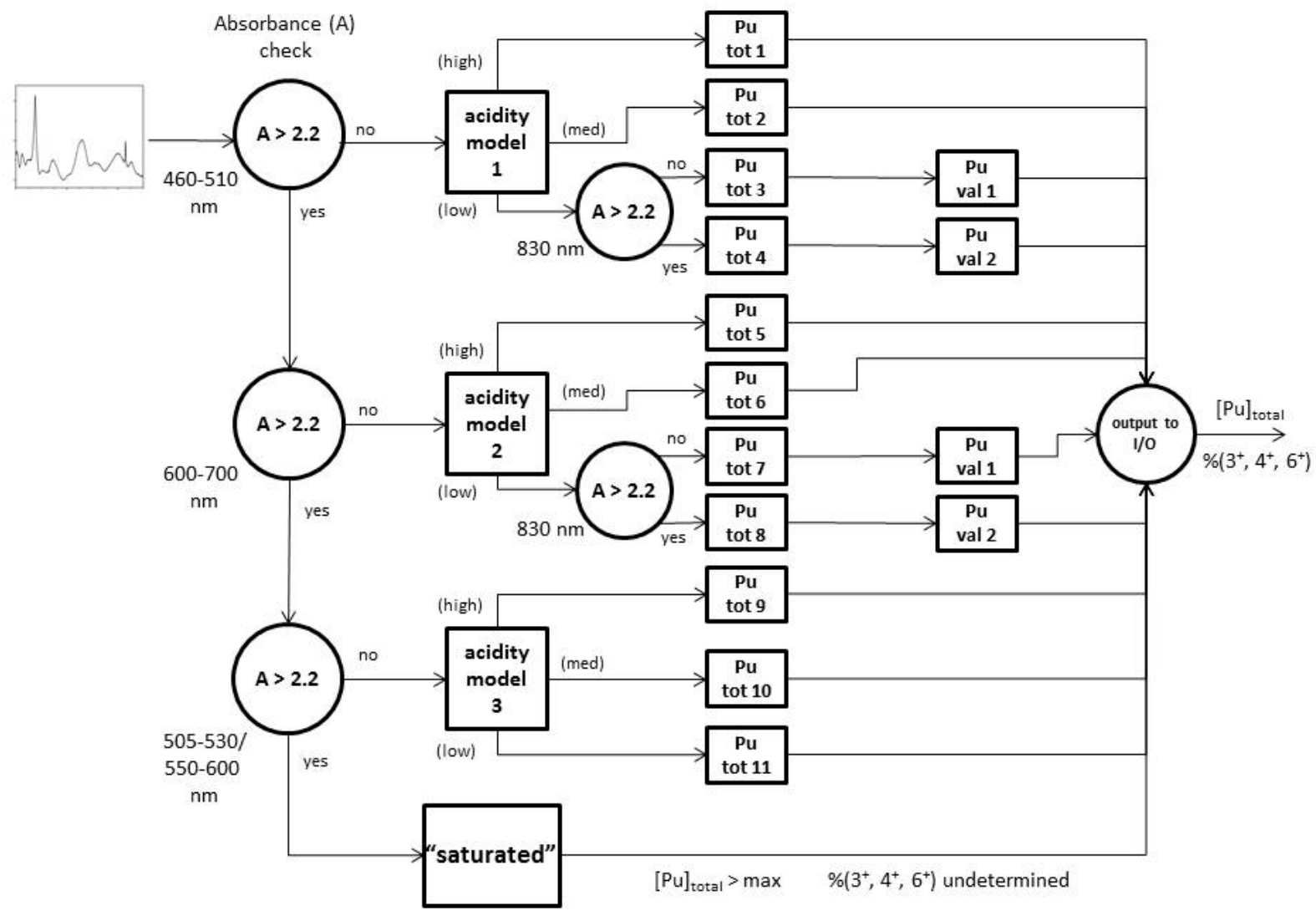

Figure 3-3. Decision tree for model subsystem.

Assuming that the spectrum is not saturated, the next decision is based on solution acidity. Each absorbance region has an acidity model, which only uses acceptable wavelengths to estimate the nitric acid concentration. Results are binned into three regions: high (6-9 M), medium (2-6 M) and low (0.3-2 M). If low acidity is indicated, a further absorption test is applied based on the possibility of disproportionation. The primary absorbance peak for $\mathrm{Pu}^{6+}$ at $830 \mathrm{~nm}$ is very strong, and there are some scenarios in which it exceeds $\mathrm{A}=2.2$ even if the main $\mathrm{Pu}^{4+}$ peak does not. ${ }^{\mathrm{a}}$ Note that for the high absorbance case, the check assumes that $\mathrm{A}(830 \mathrm{~nm})$ already exceeds 2.2. There is not enough spectral

\footnotetext{
${ }^{\mathrm{a}}$ The acidity classification models do not use the $830 \mathrm{~nm}$ peak, thus the $830 \mathrm{~nm}$ absorbance check is not required at that stage.
} 
information to assess valence state distribution, and thus that evaluation is not made for the high absorbance case.

At this point, the pathway through the decision tree has been determined. There are a total of 11 [ $\left.\mathrm{Pu}_{\text {total }}\right]$ models that may be accessed. For the low and medium absorbance cases, there are two low acid pathways, each of which has its own subsequent Pu valence model (labeled "Pu val 1" and "Pu val 2" in Figure 3-3). If $\mathrm{A}(830 \mathrm{~nm})<2.2$, then $\left[\mathrm{Pu}^{6+}\right]$ is measured directly, $\left[\mathrm{Pu}^{3+}\right]$ is assumed to be twice that value per the disproportionation reaction equation, and $\left[\mathrm{Pu}^{4+}\right]=\left[\mathrm{Pu}_{\text {total }}\right]-3\left[\mathrm{Pu}^{6+}\right]$. For $\mathrm{A}(830 \mathrm{~nm}) \geq 2.2,\left[\mathrm{Pu}^{3+}\right]$ is measured directly, $\left[\mathrm{Pu}^{6+}\right]={ }_{1}^{1} 2\left[\mathrm{Pu}^{3+}\right]$, and $\left[\mathrm{Pu}^{4+}\right]=\left[\mathrm{Pu}_{\text {total }}\right]-{ }_{3}^{3}{ }_{2}\left[\mathrm{Pu}^{3+}\right]$. For the medium and high acidity cases, $\left[\mathrm{Pu}^{3+}\right]=\left[\mathrm{Pu}^{6+}\right]=0$ and $\left[\mathrm{Pu}^{4+}\right]=\left[\mathrm{Pu}_{\text {total }}\right]$.

In addition to concentration values, all prediction models for total and individual Pu species will generate an evaluation of the spectral fit residuals. This value is a measure of how closely the input spectrum resembled the spectra generated in the laboratory and used to make the prediction models. The observation of large residuals typically indicates the presence of interfering species in the solution, although high solution turbulence can also be a cause. If the instantaneous residuals exceed the typical model residual by a certain factor (typically 100x), the "Confidence" indicator is illuminated. ${ }^{\text {a }}$

Once $\left[\mathrm{Pu}_{\mathrm{tota}}\right]$ and the concentrations of the individual oxidation states have been determined, the values, as well as the high residual status flag, are passed to the main module.

\subsubsection{Input/output subsystem}

The Input/Output (I/O) subsystem is responsible for allowing communication between the spectrophotometer and the facility digital control system (DCS). It receives input from the main module describing total plutonium and plutonium oxidation state concentrations and alarm status, converts the values to a form that can be accepted by an Opto 22 controller, and passes the values to the controller over a dedicated Ethernet interface. Specifically, concentrations (in units of g/L) are converted to a 4-20 $\mathrm{mA}$ current level, and alarms are converted to a voltage reading ( $24 \mathrm{~V}=$ alarm off, $0 \mathrm{~V}=$ alarm on). The communication to the Opto 22 also includes addressing information that ensures that values are passed to the correct DCS channel. Setup parameters, which include addressing information and the conversion from concentration units to current, are defined in a configuration file (IO-Setup.ini) that is read during instrument startup. As with other operational parameters, these settings can be accessed during operation from a password-protected screen in the main program. Other $\mathrm{I} / \mathrm{O}$ functions include trapping errors if there is a communication problem with the Opto 22 or if an invalid address or value is specified, and passing a flag to the main program if the state of a digital (alarm) input channel changes.

\subsubsection{Instrument operation, calibration and troubleshooting}

The program is designed to run after power-up and computer boot-up. It is activated by double-clicking the NGS-Main icon on the Desktop environment in Windows. Further user interaction is only required for calibration and testing. The program is not loaded into the boot-up batch file so that Windows can load all the necessary drivers before the program is called.

Appendix B provides work instructions for performing the instrument calibration, including setting the zero for the instrument, verifying current output, and verifying instrument output for a check filter. The generalized sequence of actions is:

\footnotetext{
${ }^{\mathrm{a}}$ In this situation, the instrument will continue to generate $\left[\mathrm{Pu}_{\text {total }}\right]$ readings. Historically, certain activities such as valve operation have been known to generate air bubbles in the colorimeter flow cell, with the bubbles being cleared by continued solution flow. SRNL should be consulted if the Confidence light persists for a longer period of time than expected for these conditions.
} 
- Enter the (password-protected) calibration mode by clicking a button on the main display screen of the program

- Re-zero the instrument by acquiring a blank spectrum

0 This action presumes that a blank solution is in the flow cell

- Run absorbance checks with traceable standards

o The user will be guided through this process in a specialized window on the screen. The program will perform the calculations to determine if the checks are passed and allow the user to generate a report to supplement entries in the calibration procedure.

- Verify current output for the zero (4 mA) and span (20 mA) conditions, through a check button on the main screen.

- Verify complete system function by activating a check filter (similar to the span filter operation on the colorimeter, with the exception that the filter reading is not used to calibrate the instrument, only to verify operation)

Each instrument display panel has several labeled indicator lights (yellow and red) which will illuminate if certain conditions occur. Also, an error signal can be generated from the I/O module or (in a noncritical case) may be displayed on the main screen of the computer. Table 3-1 indicates possible causes for each alarm condition and steps that users can take to address the causes. For some rare events, SRNL troubleshooting will be required. 
Table 3-1. Trouble alarms, causes, and response.

\begin{tabular}{|c|c|c|}
\hline Error & Cause & Response \\
\hline $\begin{array}{l}\text { - Xe fault } \\
\text { - Trouble }\end{array}$ & $\begin{array}{l}\text { The most likely causes are misalignment of the } \\
\text { Xe lamp (preventing the expected amount of } \\
\text { light from this lamp from reaching the reference } \\
\text { detector) or degraded performance/failure of the } \\
\text { lamp. Less likely causes include a failure of the } \\
\text { internal fiber leading to the reference } \\
\text { spectrometer and a failure of the reference } \\
\text { spectrometer itself. }\end{array}$ & $\begin{array}{l}\text { Perform the lamp alignment procedure. If the } \\
\text { Xe flash lamp is broken, it will not be possible } \\
\text { to finish the procedure. Other causes require } \\
\text { SRNL attention. }\end{array}$ \\
\hline $\begin{array}{l}\text { - HT fault } \\
\text { - Trouble }\end{array}$ & $\begin{array}{l}\text { The most likely causes are misalignment of the } \\
\text { tungsten-halogen lamp (preventing the expected } \\
\text { amount of light from this lamp from reaching } \\
\text { the reference detector) or degraded } \\
\text { performance/failure of the lamp. Less likely } \\
\text { causes include a failure of the internal fiber } \\
\text { leading to the reference spectrometer and a } \\
\text { failure of the reference spectrometer itself. }\end{array}$ & $\begin{array}{l}\text { Perform the lamp alignment procedure. If the } \\
\text { tungsten-halogen lamp is broken, it will not be } \\
\text { possible to finish the procedure. Other causes } \\
\text { require SRNL attention. }\end{array}$ \\
\hline $\begin{array}{l}\text { Confidence } \\
\text { (no Trouble } \\
\text { light) }\end{array}$ & $\begin{array}{l}\text { There is adequate light throughput but high } \\
\text { variability in the light levels incident on the } \\
\text { signal spectrometer. The variability is caused } \\
\text { by fluctuations associated with the presence of } \\
\text { bubbles in the flow cell. The variability } \\
\text { threshold is configurable in the software } \\
\text { (configuration file). }\end{array}$ & $\begin{array}{l}\text { This condition arises from the flow cell and } \\
\text { cannot be addressed in the spectrometer apart } \\
\text { from the changing the threshold value. The } \\
\text { appropriate response is to wait for the bubbles } \\
\text { to dissipate. Testing with the colorimeters } \\
\text { indicated that bubbles tend to be associated with } \\
\text { valve operation and are temporary. }\end{array}$ \\
\hline - Trouble & $\begin{array}{l}\text { There are a number of causes of a trouble light } \\
\text { unaccompanied by a yellow warning light. } \\
\text { These include: drift of the wavelength } \\
\text { calibration for the spectrometers; light } \\
\text { intensities are too high or too low; Pu reading } \\
\text { has drifted too low below zero; spectrometer is } \\
\text { not responding to main module (“timed out”); } \\
\text { program cannot find a file or access the disk. }\end{array}$ & $\begin{array}{l}\text { More detailed information can often be found } \\
\text { on the status screen for the instrument. If light } \\
\text { intensity is thought to be an issue, the lamp } \\
\text { alignment procedure should be performed. Low } \\
\text { light intensity on the sample spectrometer can } \\
\text { also arise due to fiber or flow cell throughput } \\
\text { issues. Broken fibers or flow cell can be } \\
\text { examined by fiber manipulation (for example } \\
\text { disconnecting the fibers from the flow cell and } \\
\text { jumpering them together to bypass the cell). } \\
\text { High Pu in the flow cell (for example during the } \\
\text { heart cut) can also cause a low light intensity. } \\
\text { As with the colorimeters, this condition will } \\
\text { pass as the elution transitions to the tails cut. } \\
\text { Low cell throughput when Pu is not present can } \\
\text { also arise from a trapped bubble. Other errors } \\
\text { are expected to be rare; SRNL should be } \\
\text { consulted in these cases. }\end{array}$ \\
\hline I/O watchdog & $\begin{array}{l}\text { Lack of communication between the I/O module } \\
\text { and the spectrophotometer module (queries } \\
\text { every } 4 \text { seconds). }\end{array}$ & $\begin{array}{l}\text { Very rare occurrence. The spectrophotometer } \\
\text { should be restarted. }\end{array}$ \\
\hline $\begin{array}{l}\text { Display panel } \\
\text { notification } \\
\text { (no warning } \\
\text { light) }\end{array}$ & High fit residuals & $\begin{array}{l}\text { These may occur if the process conditions are } \\
\text { outside the Pu calibration range (for example, } \\
\text { an unexpected light-absorbing species in } \\
\text { solution), or if the wrong model is applied (if } \\
\text { the acidity is miscalculated beyond the assumed } \\
\text { error described in Section x.) High fit residuals } \\
\text { are not automatically indicative of measurement } \\
\text { error. If the display persists, notify SRNL. }\end{array}$ \\
\hline
\end{tabular}




\subsection{Calibrations}

This section begins with a discussion of how the absorbance checks of the spectrophotometer with calibrated standards is a viable alternative to a field calibration done by measuring plutonium standards. This relationship is important because, once the spectrophotometers have been installed in HB-Line, they cannot be validated using known Pu solutions. Standards cannot be poured into flow cells, as is done with uranium standards for the $\mathrm{H}$ Canyon colorimeters, and cuvettes of plutonium solution cannot be dropped into the sample holders on the instrument itself due to radiological concerns. However, traceable absorbance standards can be measured to confirm the fundamental accuracy of the instrument. The next section describes the statistical methods used to create the plutonium calibration models which convert spectra to concentration. Subsequent sections discuss specifics of calibrations for nitric acid concentration (used to classify spectra as described in Section 3.4.2), total plutonium concentration, and plutonium oxidation state distribution.

\subsection{Spectrometer calibration.}

The approach for calibrating the spectrophotometers for $\mathrm{Pu}$ measurements and assuring, via field measurements, that the instruments are operating properly is somewhat different than what is used for the colorimeters. A detailed comparison of the two methods will be useful in demonstrating that the spectrophotometer can be reliably evaluated for proper operation.

Colorimeter calibration is based on the assumption that the analyte concentration is linearly proportional to the signal difference between the measure and reference detectors. As a line can be defined by identifying two points that lie on it, the calibration is defined by establishing zero and span points. The zero point is established with a blank solution (not containing $\mathrm{Pu}$ ) in the flow cell. The span is ideally established with a solution containing the highest possible amount of $\mathrm{Pu}$, so that all subsequent measurements will be interpolated between the two responses. In H Canyon, where colorimeters are used for $\mathrm{U}$ monitoring, it is possible to introduce a span solution into the flow cell, and it is likewise possible to measure solutions with intermediate $U$ concentrations to confirm the linearity of response within the range. In HB-Line, direct measurement of Pu standard solutions is not possible without moving the instrument to a laboratory. Instead, an internal span filter (a slab of colored glass) simulates a Pu solution and serves as a secondary standard. The apparent Pu concentration of the span filter is determined by laboratory measurements whereby the colorimeter is calibrated to Pu standards and then the reading for the span filter is noted.

This approach is an empirical calibration, as the intrinsic optical properties of the bandpass filters for the reference and measure detectors, as well as the span filter, are never measured. Likewise, other properties of the colorimeter which affect its ability to accurately measure light intensity are not checked. The colorimeter is treated as a black box which generates a reading, and the performance of the instrument is defined entirely by its ability to reproduce the concentration of $\mathrm{Pu}$ standards. Because the optical properties of the filters and the instrument performance change over time, the apparent Pu concentration of the span filter has changed with each yearly calibration.

As the spectrophotometers are directly replacing the colorimeters, the restrictions against directly measuring Pu standards in the field still hold. However, the spectrophotometer is not a black box instrument. One can measure the accuracy of the instrument with respect to measuring both the incident wavelength and intensity, and hence the solution absorbance, using traceable standards and fundamental constants such as the xenon emission lines in the flash lamp. If the wavelength and absorbances of the standards span the values measured in the plutonium absorbance spectra, the latter can be assumed to be valid. The validity of the prediction models (total plutonium, plutonium oxidation state, and nitric acid) 
are also based on certification of the instruments used to measure the spectra of the calibration standards. If the spectra of the standards can be considered fundamentally accurate, then the prediction models are also accurate and it is valid to apply them to subsequent spectra acquired in the field. This is true even if the spectra are obtained with a different instrument. The uncertainty associated with the reported concentration will reflect the variance between the different instruments while still being able to meet the tolerances of the wavelength and absorbance standards. As long as the standards themselves do not change, or are recertified, the continued ability of the spectrometers to measure those standards is equivalent to their being able to measure plutonium accurately.

\subsection{Pu calibration - overview.}

The colorimeters are designed so that the measurement of an absorbance can be translated to a $\mathrm{Pu}$ concentration with a simple conversion factor. This relationship comes directly from the Beer-Lambert Law (Equation 1), where $A$ and $c$ are directly proportional to each other. The calculation is made explicitly for the raffinate colorimeter, which displays absorbance and requires conversion to concentration. The conversion is done internally for the NK-1 and NK-3 colorimeters, which report concentration units. For the spectrophotometers, the conversion of the absorbance spectrum to acidity, total Pu concentration, or Pu oxidation state distribution is more complicated. The mathematical methods for the calibration are well known [16-17] and widely used in many industrial monitoring applications. [18-19] They are the basis for other absorbance instrumentation in use at Savannah River Site, including H Canyon spectrophotometers [14] and diode array spectroscopy methods in Analytical Laboratories [20]. The references cited and many other works allow an understanding of the methods at any desired level of mathematical rigor. The explanation provided here gives a qualitative picture that supports an understanding of the development work that was done to generate a Pu calibration.

The calibration for total plutonium concentration is based on absorbance spectra recorded for a set of standard solutions which span the range of conditions appropriate for the AFS-2 process. The variables considered include total Pu concentration ( 0 - $7 \mathrm{~g} / \mathrm{L})$, acidity (0.3 - $9 \mathrm{M}$ nitric), solution temperature (20 $45^{\circ} \mathrm{C}$ ), and different Pu oxidation states (predominantly 4+, but also $3+$ and $6+$ arising from the low-acid disproportionation reaction). ${ }^{\mathrm{a}}$ The variations in all of these conditions lead to changes in the absorbance spectrum. The primary goal of the calibration is to determine the correlations between absorbance and total $\mathrm{Pu}$ concentration. This goal is best achieved by first determining the solution acidity with another calibration. Determination of oxidation state distribution is a secondary goal. The correlations then become the basis by which a new spectrum can be interpreted to determine the concentration of that chemical that needed to have been present to give rise to that spectrum.

The formulas that are developed to convert spectra into concentrations do not require inputs for the values of the other properties of the solution. Specifically, calibrations for total Pu concentration do not require that the concentrations of the $\mathrm{Pu}^{3+}, \mathrm{Pu}^{4+}$, and $\mathrm{Pu}^{6+}$ species be determined independently and added together. Likewise, neither the acidity (apart from specifying a particular total plutonium model) nor the temperature of the solution are represented in the formulas. The only condition which must be met is that the calibration set must have a representative distribution of these other properties.

The mathematical process used here to convert a spectrum into a measurement is generally known as Principal Component Analysis (PCA). The goal of PCA is to simplify a data set so that the statistically significant variation within the set is more easily observable. The process can be understood in a geometrical sense, by analogy to a simple example. The location of a point can be represented in threedimensional space by a vector $(x, y, z)$. Consider a series of points which are distributed along a line not coincident with the $x, y$, or $z$ axis. The position of each point along the line is associated with some

\footnotetext{
${ }^{a}$ Any process changes resulting in nitric acid concentrations outside this range will require an evaluation of potential biases in the instrument output. The calibration models may need to be redeveloped in such a case.
} 
property $p$. One could predict $p$ for any point on the line with a formula which includes terms for each axis, e.g. $p=f_{x}(x)+f_{y}(y)+f_{z}(z)$. Or, one could rotate the axes so that one of the new axes $\left(x^{\prime}\right)$ lies along the line defined by the points and $p$ is described by a single term, $p=f_{x^{\prime}}\left(x^{\prime}\right)$. All of the relevant information has been reduced from three dimensions to a single dimension, or "principal component" (PC), which is a linear combination of the three original dimensions. The other two new dimensions, $y$ ' and $z$ ', only contain noise, and can be ignored.

Likewise, an absorbance spectrum measured at $M$ distinct wavelengths is equivalent to a single point in a $M$-dimensional space $\left(x_{1}, x_{2}, \ldots x_{M}\right)$. PCA determines how to rotate the $M$ axes so that all of the meaningful variation in a collection of spectra is represented by as few PCs as possible. The first PC is calculated to minimize the total difference between itself and all of the spectra in the set. A new data set is formed which each new "spectrum" is the difference between the original spectrum and the first PC. A second PC is determined from the new data set in the same way, and the process is repeated until the residual data set only contains noise, as determined statistically. ${ }^{\text {a }}$

To this point, concentrations have not been included in the data analysis. A variant of PCA called Partial Least Squares (PLS) provides this link by incorporating standard values into the calculation. Each PC will have a constant ("score") associated with it that converts magnitude of the PC to a concentration. In PLS, the first PC and its score are chosen so that the overall error in concentration prediction is minimized. The residual spectral set is accompanied by a residual concentration vector, and both are used in the calculation of the second PC (with subsequent iterations, as required). The final model is the set of PCs and the associated scores.

Application of the model to predict the concentration of a new spectrum is relatively straightforward. The first PC is fit to the data set with a single multiplicative factor, the second PC is fit to the residual, and so forth for all PCs in the model. Each magnitude is multiplied by the appropriate score and the total is summed to provide the final concentration prediction.

Ideally, the number of PCs will be the same as the number of physical factors which are causing changes in the data set. Over the range of relevant conditions in the AFS-2 process, the number of factors is large. Scoping work done on the entire Pu calibration data set indicated that a single universal model would require 9-14 PCs. However, models with a large number of PCs are less desirable. The PCs tend to have more noise, which makes the models more susceptible to prediction errors when new spectra are analyzed. To make predictions more robust, it is often feasible to divide the calibration set into regions which individually have fewer sources of variation.

For total Pu measurements, the strongest classification can be made on the basis of acidity. Dividing the total acidity range into three sections (0.3-2 M, 2-6 $\mathrm{M}$, and 6-9 $\mathrm{M}$ ) reduces, by one, the number of $\mathrm{Pu}$ species covered by a single model. Choosing a dividing point at $2 \mathrm{M}$ also isolates the effect of disproportionation to the lowest acidity region. Thus only the 4+ oxidation state needs to be considered for the medium- and high-acidity cases. ${ }^{\mathrm{b}}$

Further classification can be obtained based on solution absorbance. Spectra can be evaluated to determine if any absorbances exceed the maximum value for the instrument, and models which only use data from valid wavelengths can be applied. Because the solutions will always be predominantly $\mathrm{Pu}^{4+}$ under process conditions, the evaluation scheme listed in Section 3.4.2 will always be valid, and thus the

\footnotetext{
${ }^{a}$ In calculating the new set of PCs, they are also chosen to be orthogonal, so that they have the necessary properties of a new coordinate system.

${ }^{\mathrm{b}}$ Oxidation of $\mathrm{Pu}^{4+}$ to $\mathrm{Pu}^{6+}$ at high acid and high temperature, which can occur in $\mathrm{H}$ Canyon dissolver tanks [27], is not within the scope of this method application. However, models which include this effect can be developed at a later date.
} 
number of models used can be restricted to the three wavelength ranges previously described. A further benefit of absorbance-based classification is that a much wider range of $\mathrm{Pu}$ concentrations can be measured than would be the case if only a single set of wavelengths were considered. The strongest absorbing peaks provide the best sensitivity to low concentrations, and the weakest peaks allow measurement of the highest concentrations.

There are costs associated with classification, but in this case they are not prohibitive. The greatest potential cost is in the time required for model development. One universal total $\mathrm{Pu}$ model and one $\mathrm{Pu}$ valence model have been replaced by three absorbance checks, three acidity models, eleven total $\mathrm{Pu}$ models, and two Pu valence models. However, optimizing an individual model is straightforward, and most of the increased effort is associated with bookkeeping (wavelength and calibration standard selection). The total number of calibration spectra and standard solutions remains the same. In practice, running the classification scheme and applying the correct model takes less than 5 seconds and is not the rate-limiting step for instrument operation. There is a potential cost associated with a mistake during classification; for example, there may be an error in total $\mathrm{Pu}$ measurement if a medium-acid model is applied to a high-acid solution. This cost is reduced by having high-accuracy acidity models and by having standards common to multiple Pu models. To implement the latter, standards with $2 \mathrm{M}$ acidity are common to low- and medium-acid models, and those with $6 \mathrm{M}$ acidity are common to medium- and highacid models. The determination of the uncertainty associated with misclassification is calculated in Section 5.0.

\subsection{Methods}

Pu calibration standards were made gravimetrically from stock solutions of Pu in nitric acid, nitric acid, and water. The Pu and acid concentrations and densities of the Pu stock solutions were determined by standard methods. ${ }^{a}$ The nitric acid stock solutions were prepared gravimetrically from a single bottle of ACS reagent grade $70 \%$ nitric acid. Portions of each stock solution were delivered into a vial on a balance controlled under the SRNL Measurement and Test Equipment (M\&TE) program. For each stock solution, the mass and density measurements allowed calculation of the volume delivered. After all stock components were delivered and mixed, the density of each standard solution was measured with a Parr DMA 35N densitometer. The densitometer was verified with distilled water before and after each use to an accuracy of $\pm 0.0003 \mathrm{~g} / \mathrm{mL}$. From the total mass and density of each standard, a total volume was determined, which allowed calculation of the concentrations of each component (Pu and nitric acid) in the standard. The uncertainties in these concentrations are based on the propagation of uncertainties from the original stock solution concentrations and in the uncertainties in the M\&TE (balance and densitometer). Uncertainties $(1 \sigma)$ for the standards range from $0.38-0.54 \%$, and are slightly higher for lower concentrations. For simplicity, the largest value of $0.54 \%$ is applied to all standards.

Figure 4- 1 shows the range of $\mathrm{Pu}$ and nitric acid concentrations covered by the standard solution set. Nitric acid concentrations $(0.3-9 \mathrm{M})$ were chosen to exceed the expected range of values during processing. Pu concentrations were chosen to obtain as wide a range of absorbance values as possible for a $1 \mathrm{~cm}$ path length. Additional standards were made with plutonium concentrations of 0.12 and $0.5 \mathrm{~g} / \mathrm{L}$, but these only generated a weak, noisy absorbance signal with the $1 \mathrm{~cm}$ cuvettes and were excluded from subsequent data analysis. Cuvettes were made of quartz for the largest transmission range and were sealed with polymer-coated screw caps to prevent concentration changes due to evaporation. Temperature dependence of selected standards (noted in Figure 4-1 with circles) was determined by heating the sealed cuvettes in a heated block to $60{ }^{\circ} \mathrm{C}$. One at a time, standards were removed, immediately inserted into a cuvette holder, and spectra were taken continually as the solutions cooled until no further spectral changes

\footnotetext{
${ }^{a} \mathrm{Pu}$ was determined by Analytical Laboratories using coulometry to an uncertainty of $0.35 \%$ (corrected for iron concentration, LIMS\# 200678016 and 200678029). Nitric acid was determined by titration in SRNL (Method ADS-1206), to an uncertainty of $10 \%$.
} 
were observed and the solutions had apparently reached room temperature. Temperatures were not explicitly measured but based on the rate of spectral change and the time taken between moving the cuvettes from the heater block and starting measurements, the highest temperature range is estimated to be $45-50{ }^{\circ} \mathrm{C}$.

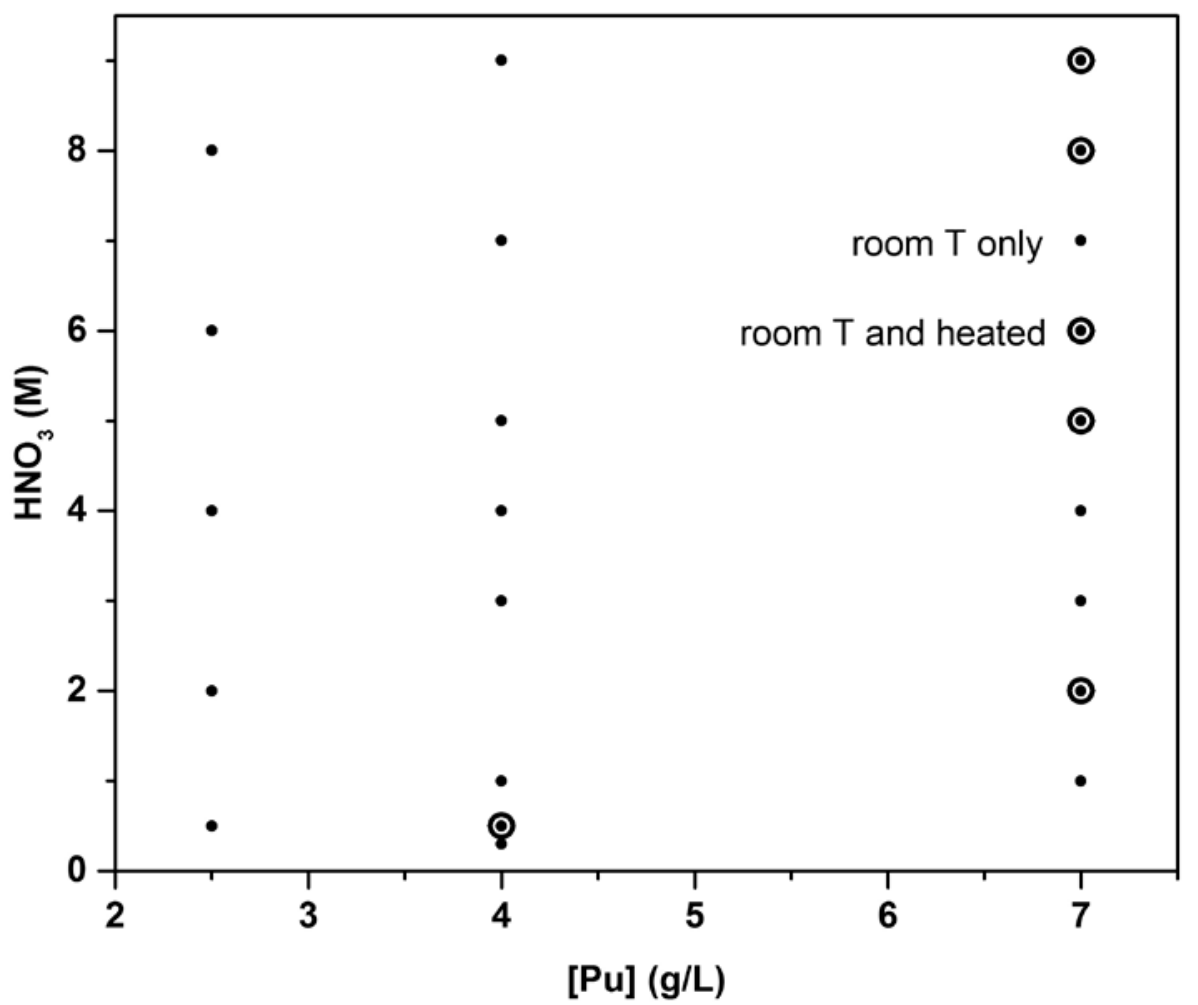

Figure 4-1. Concentrations of calibration standards.

All standard solutions were initially prepared with $\mathrm{Pu}$ in the $4+$ oxidation state. For acidities $\leq 2 \mathrm{M}$, disproportionation was allowed to progress naturally. Because the cuvettes were sealed, the total $\mathrm{Pu}$ concentration remained constant. For the models that predict total Pu concentration, the actual amount of disproportionation does not figure into the calibration. The methods used to estimate the distribution of $\mathrm{Pu}$ valence states are discussed in Section 4.6.

\subsection{Pu calibration -acidity classification models.}

Prediction of the nitric acid content in the model is based on the shapes of the absorption peaks, as shown in Figure 1-1. The most prominent shape change is for the $\mathrm{Pu}^{4+}$ absorbance peak near $475-490 \mathrm{~nm}$. At lower acidities, the peak is sharp and is centered at $475 \mathrm{~nm}$. As acidity increases, $\mathrm{Pu}^{4+}$ species with increasing nitrate anions become prevalent. The observed peak becomes the sum of the peaks of the individual Pu nitrate species, with the effect of shifting the center to longer wavelengths and making the peak broader. The equilibrium between the $\mathrm{Pu}$ nitrate species, and thus the absorbance spectrum, is independent of total Pu concentration for these process conditions. [21] Since the overall magnitude of the spectrum is dependent on $\mathrm{Pu}$ concentration, normalizing the spectra to unit area allows the effects of shape change to be isolated from the effects of Pu concentration.

Table 4-1 summarizes the three acidity classification models that are used in the analysis scheme. The top portion of the table describes the data preprocessing methods used. Preprocessing improves data 
quality and can enhance the parts of the signal that correlate to concentration changes. The terminology used here is consistent with that used in the data analysis literature and software. X-block refers to the spectral data set, and Y-block refers to the nitric acid concentrations. "SG(pts, poly, order)" refers to the Savitzky-Golay method for data smoothing and calculation of derivatives [22], with pts referring to the width of the smoothing window (a higher value indicating more smoothing), poly the order of the polynomial used to simulate the data within the window, and order the order of the derivative calculated (e.g. 2 = second derivative). "Mean centering" subtracts the average of the entire data block and is used to emphasize the relative variation of the data set. "1-norm" is area normalization as referred to in PLS_Toolbox. Cross-validation refers to the method by which the soundness of the calibration data set is checked. The calibration set is divided into two groups. A model is built on one group and applied to the other group. If the calibration set data is sound and consistent, the cross-validation prediction error (root mean-square error of cross-validation, RMSECV) will be similar to the self-prediction error when the entire data set is used (root mean-square error of calibration, RMSEC). The RMSECV also tends to give a more representative measure of the prediction error when the model is used on later data. The RMSECV is the value which is used when reporting the $1 \sigma$ uncertainty of the model and in calculating the overall uncertainty of the monitor.

Table 4-1. Parameters for acid classification models.

\begin{tabular}{|c|c|c|c|}
\hline Model name & Puclass01d & Puclass02b & Puclass03c \\
\hline Absorbance case & Low & Medium & High \\
\hline Wavelength range & $460-750 \mathrm{~nm}$ & $580-720 \mathrm{~nm}$ & $490-530$ \& 560-620 nm \\
\hline Pre-processing (X-block) & $\begin{array}{c}\text { SV(151,5,2) } \\
\text { Absolute value } \\
\text { 1-norm } \\
\text { Mean center }\end{array}$ & $\begin{array}{c}\text { SV(121,5,2) } \\
\text { Absolute value } \\
\text { 1-norm } \\
\text { Mean center }\end{array}$ & $\begin{array}{c}\text { SV(91,5,1) } \\
\text { Absolute value } \\
\text { 1-norm } \\
\text { Mean center }\end{array}$ \\
\hline Pre-processing (Y-block) & Mean center & Mean center & Mean center \\
\hline Cross-validation & $\begin{array}{c}10 \text { random subsets } \\
\text { 3 iterations }\end{array}$ & $\begin{array}{c}10 \text { random subsets } \\
\text { 3 iterations }\end{array}$ & $\begin{array}{c}10 \text { random subsets } \\
3 \text { iterations }\end{array}$ \\
\hline \# PCs & 9 & 6 & 6 \\
\hline RMSEC (M) & 0.12 & 0.16 & 0.20 \\
\hline RMSECV (M) & 0.14 & 0.18 & 22 \\
\hline $\begin{array}{c}\text { Estimated signal-to-noise } \\
\text { ratio of last PC }\end{array}$ & 4 & 21 & 0.995 \\
\hline $\begin{array}{c}\text { CV Correlation coefficient } \\
\text { (R) }\end{array}$ & 0.998 & 0.996 & -0.001 \\
\hline CV Bias (M) & 0.005 & 0.001 & 22 \\
\hline
\end{tabular}

Model performance criteria are given in the lower half of the table. In addition to the RMSEC and RMSECV are given the estimated signal-to-noise ratio of the last PC, the correlation coefficient $\left(\mathrm{R}^{2}\right)$, and the bias of the cross-validation predictions. $\mathrm{R}^{2}$ should be close to 1 to indicate a linear relationship between known and predicted concentrations, and the bias should be close to 0 to indicate that there are no offsets.

Comments on the models, individually or as a group, are as follows.

- All three models predict solution acidity with sufficient accuracy for accurate classification as low, medium, or high acidity. The error for each model increases as less spectral information becomes available. The left panels of Figure 4-2 through Figure 4-4 show the spread of predicted acidities for the three absorbance cases as function of actual solution acidity. The absolute prediction error is consistent across the acidity range. The results indicate that acid 
misclassification errors will be restricted to samples within a narrow region around the $2 \mathrm{M}$ and 6 M boundaries.

- Further validation is provided by applying the model to an independent set of measurements. As a new set of standards was not available, the validation data set was obtained by measuring the original standards with several different spectrophotometers of the same design. Summaries of the results for the three absorbance cases are shown in Figure 4-2 through Figure 4-4. Each point represents the mean error for measurement of a single standard with 8 different instruments, with the $1 \sigma$ standard deviation represented by error bars. The straight line (slope $=1$ ) is to guide the eye. The RMSEC for the three data sets are $0.26,0.25$, and $0.33 \mathrm{M}$, respectively, which are consistent with the RMSECVs obtained from calibration and further confirm the soundness of the acidity prediction model.

- The high absorbance model uses first derivative processing, in contrast to the second derivative processing used for the low and medium absorbance cases. This approach is taken because the limited wavelength region of the high absorbance case does not include any discrete peaks and the second derivatives tend to suppress the differences between spectra. Second-derivative models gave poor performance regardless of the parameters chosen.

- The acid classification models rely on spectral changes due to different amounts of Pu nitrate species. Pu must be present in the solution for these models to give meaningful results. The models are not designed to be sensitive to the differences between low- and high-acid solutions without $\mathrm{Pu}$ (such as recon or decon solutions).

- Figure 4-5 demonstrates the need to represent temperature variation in the calibration set. Samples 1-112 represent spectra from 11 standards as they cooled from $50{ }^{\circ} \mathrm{C}$ (lower sample no.) to room temperature. The expected values are plotted as black solid dots. The values plotted as red open circles are results obtained based on a model which only included room temperature spectra. The prediction errors for higher temperature samples are severe. Note that low acid solutions $(\leq 2 \mathrm{M})$ would be identified as having medium acidity. If that happened, the classification flow chart would not invoke any total plutonium models that included disproportionation, and significant measurement errors would result. The model results including temperature variation in the calibration set are shown as blue open triangles. In contrast to the room temperature models, these results have almost no temperature dependence and are clearly much more accurate in terms of proper classification. 
SRNL-STI-2015-00454

Revision 0

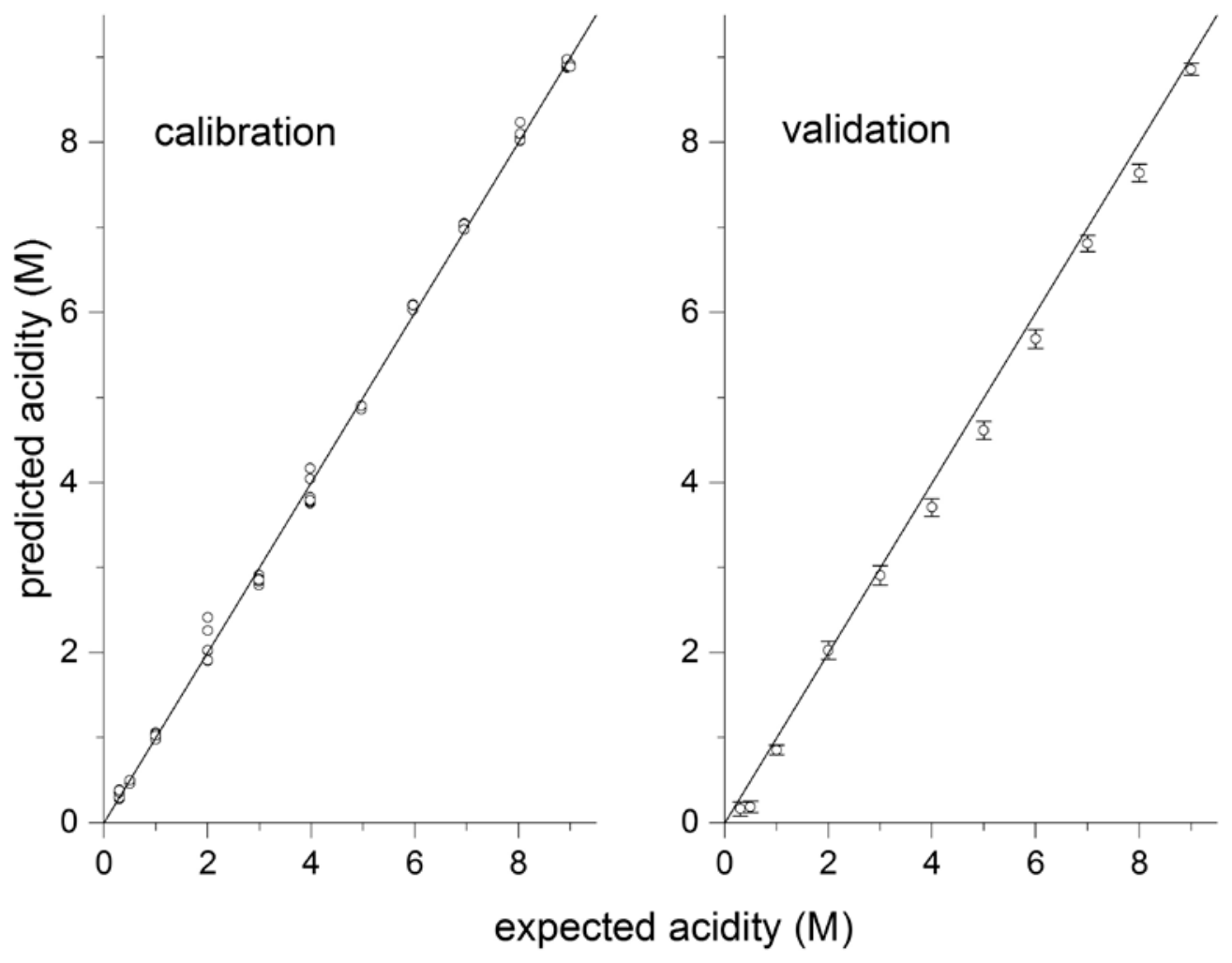

Figure 4-2. Results of low absorbance acid prediction model. 


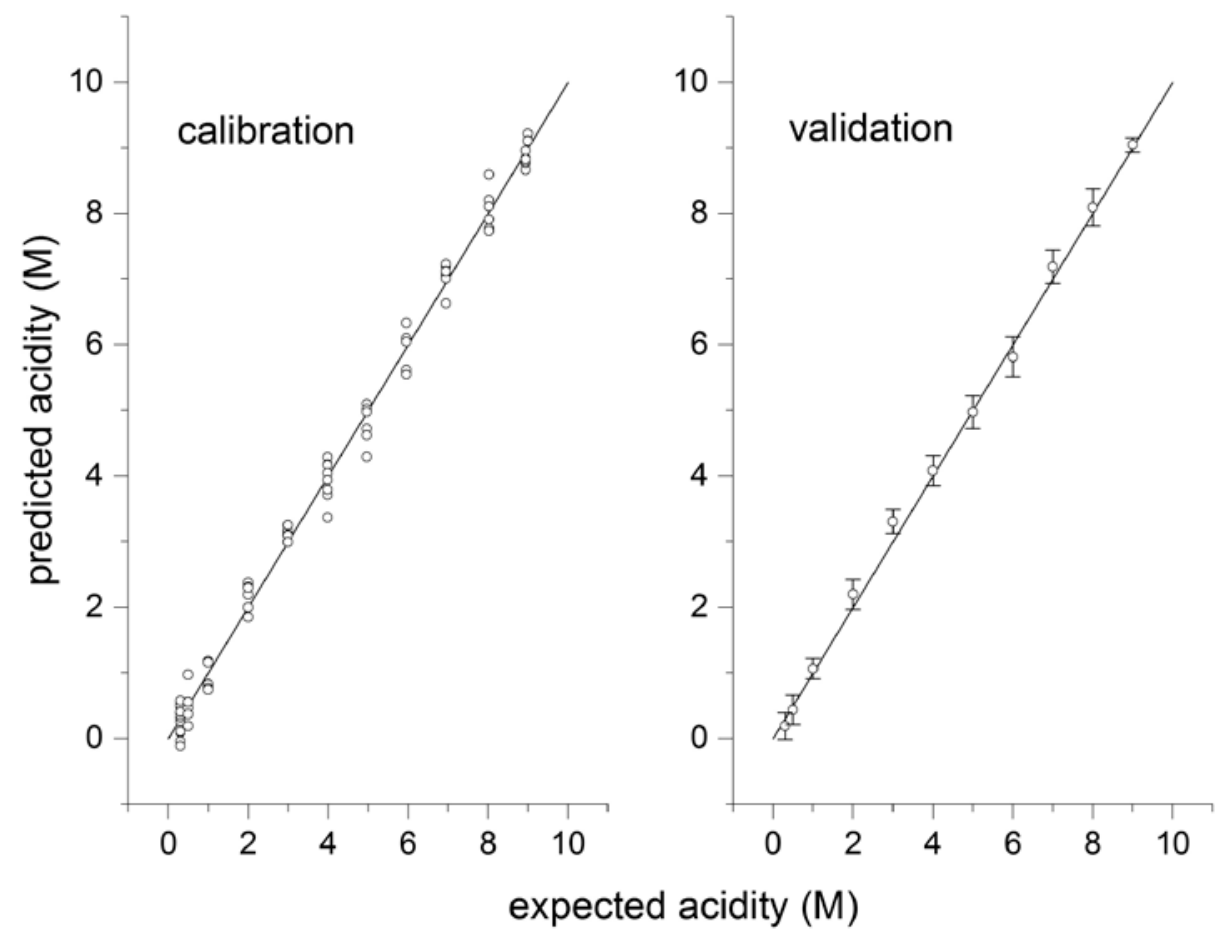

Figure 4-3. Results of medium absorbance acid prediction model.

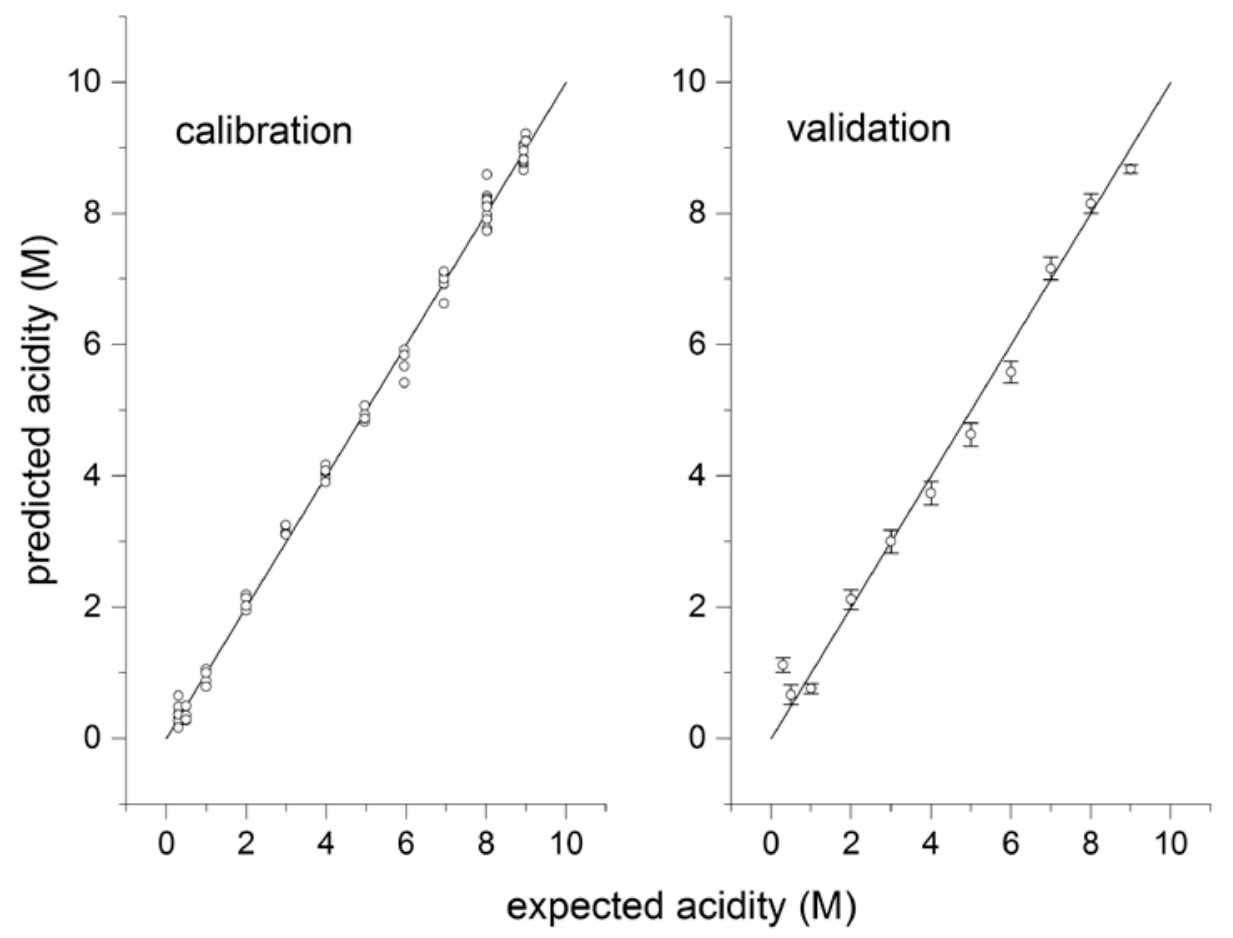

Figure 4-4. Results of high absorbance acid prediction model. 
SRNL-STI-2015-00454

Revision 0

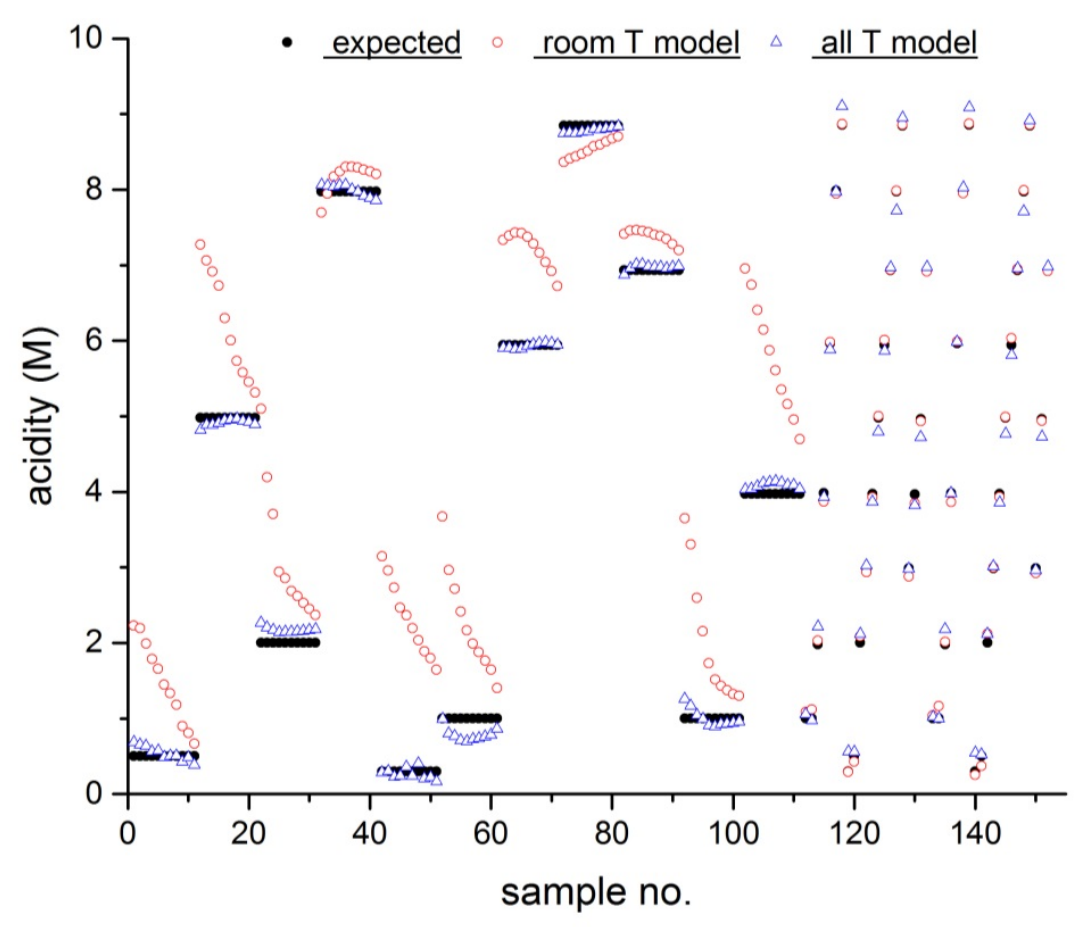

Figure 4-5. Temperature dependence of acid classification results.

\subsection{Pu calibration - total Pu models.}

As indicated by Figure 3-3, there are 11 prediction models for total $\mathrm{Pu}$, which are selected based upon solution absorbance and acidity. Table 4-2 through Table 4-4 provide the relevant descriptions of the models and their performance. The models were generated using appropriate subsets of the total calibration solution set, based on both absorbance and acidity. If a solution had an absorbance greater than 2.2 at any wavelength which would be included in the model, that spectrum was excluded from the calibration set. All solutions with acidity at or below $2 \mathrm{M}$ were grouped to isolate disproportionation into one acid region. The medium/high acidity region threshold of $6 \mathrm{M}$ was chosen based on the appearance of a higher plutonium nitrate species at $\sim 7 \mathrm{M}$. The various parameters and evaluation criteria in the tables are the same as for the acid classification models. The limit of detection is given as $3 \mathrm{x}$ the standard deviation of results obtained for blank spectra. It is only listed for the low absorbance models, since these are the models that will be invoked at low Pu concentrations. The limits of detection are also scaled for measurement in the 1 " flow cell used in the field, as opposed to the $1 \mathrm{~cm}$ cuvettes used for the standards.

Comments on the models are as follows.

- Figure 4-6 through Figure 4-8 show residual plots for the models and indicate a high degree of linearity (which is also supported by the $\mathrm{R}^{2}$ values). For each model, the left panel represents the self-predictions of the model based on the cross-validation. Each cluster of points represents a number of solutions with the same Pu concentration but different acidities and temperatures. The straight line is for guidance only (slope $=1$ ), and is not a fit to the data. The right panel for each model shows validation results based on using the model to analyze measurements obtained with multiple spectrometers. The error bars indicate $\pm 2 \sigma$ residuals for each standard. Note that the $\mathrm{Pu}$ concentration in these figures is scaled for the $1 \mathrm{~cm}$ cuvettes. 
Table 4-2. Total [Pu] model parameters, 6-9 $\mathrm{M}$ acid cases.

\begin{tabular}{|c|c|c|c|}
\hline Model name & R1Putot10 & R2Putot11 & R3Putot12 \\
\hline Absorbance case & Low & Medium & High \\
\hline Wavelength range & $420-600 \mathrm{~nm}$ & $525-730 \mathrm{~nm}$ & $490-530$ \& 560-620 nm \\
\hline Pre-processing (X-block) & $\begin{array}{c}\text { SG(201,5,2) } \\
\text { Mean Center }\end{array}$ & $\begin{array}{c}\text { SG(201,5,2) } \\
\text { Mean Center }\end{array}$ & $\begin{array}{c}\text { SG(61,5,1) } \\
\text { Mean Center }\end{array}$ \\
\hline Pre-processing (Y-block) & Mean Center & Mean Center & Mean Center \\
\hline Cross-validation & $\begin{array}{c}\text { 8 Random subsets } \\
\text { 3 iterations }\end{array}$ & $\begin{array}{c}\text { 8 Random subsets } \\
\text { 3 iterations }\end{array}$ & $\begin{array}{c}\text { 8 Random subsets } \\
\text { 3 iterations }\end{array}$ \\
\hline \# PCs & 4 & 4 & 4 \\
\hline RMSEC (g/L) & 0.021 & 0.039 & 0.029 \\
\hline RMSECV (g/L) & 0.023 & 0.046 & 0.032 \\
\hline $\begin{array}{c}\text { Estimated signal-to-noise } \\
\text { ratio of last PC }\end{array}$ & 7 & 2.3 & 0.9999 \\
\hline $\begin{array}{c}\text { CV Correlation coefficient } \\
\text { (R2) }\end{array}$ & 0.9999 & 0.9998 & -0.0003 \\
\hline CV Bias (M) & 0.0001 & -0.0014 & --- \\
\hline $\begin{array}{c}\text { Limit of detection (g/L) } \\
\text { (for 1" flow cell) }\end{array}$ & 0.01 & --- & \\
\hline \hline
\end{tabular}

Table 4-3. Total [Pu] model parameters, 2-6 $\mathrm{M}$ acid cases.

\begin{tabular}{||c|c|c|c||}
\hline Model name & R1Putot02 & R2Putot06 & R3Putot04 \\
\hline Absorbance case & Low & Medium & High \\
\hline Wavelength range & $450-525 \& 810-860 \mathrm{~nm}$ & $525-670 \mathrm{~nm}$ & $505-530$ \& 550-600 nm \\
\hline Pre-processing (X-block) & $\begin{array}{c}\text { SV(151,5,2) } \\
\text { Mean Center }\end{array}$ & $\begin{array}{c}\text { SV(201,5,2) } \\
\text { Mean Center }\end{array}$ & $\begin{array}{c}\text { SV(61,5,1) } \\
\text { Mean Center }\end{array}$ \\
\hline Pre-processing (Y-block) & Mean Center & Mean Center & Mean Center \\
\hline Cross-validation & $\begin{array}{c}\text { 8 Random subsets } \\
\text { 3 iterations }\end{array}$ & $\begin{array}{c}\text { 8 Random subsets } \\
\text { 3 iterations }\end{array}$ & $\begin{array}{c}\text { 8 Random subsets } \\
\text { 3 iterations }\end{array}$ \\
\hline \# PCs & 4 & 5 & 4 \\
\hline RMSEC (g/L) & 0.060 & 0.040 & 0.064 \\
\hline RMSECV (g/L) & 0.067 & 0.047 & 0.072 \\
\hline $\begin{array}{c}\text { Estimated signal-to-noise } \\
\text { ratio of last PC }\end{array}$ & 10 & 7 & 26 \\
\hline $\begin{array}{c}\text { CV Correlation coefficient } \\
\text { (R2) }\end{array}$ & 0.9995 & 0.9998 & 0.9994 \\
\hline CV Bias (M) & -0.0013 & -0.0012 & -0.0004 \\
\hline $\begin{array}{c}\text { Limit of detection (g/L) } \\
\text { (for 1” flow cell) }\end{array}$ & 0.01 & --- & -- \\
\hline \hline
\end{tabular}

- As with the acidity prediction models, the temperature variance of the predicted results is reduced by the inclusion of spectra taken at elevated temperatures in the calibration data set. Figure 4-9 shows a representative example of the improvements, in this case for the high acidity, medium absorbance model (R2Putot11). Prediction errors for solutions at elevated temperatures are as high as $5-7 \%$ when using a model based only on room temperature data. These reduce to $1 \%$ or less with the inclusion of all the data. The direction of the remaining bias with elevated temperature is apparently random. This variation is included in the overall uncertainty for each individual model. 
Table 4-4. Total [Pu] model parameters, 0.3-2 $\mathrm{M}$ acid cases.

\begin{tabular}{|c|c|c|c|c|c|}
\hline Model name & R1Putot21 & R1Putot23 & R2Putot22 & R2Putot23 & R3Putot20 \\
\hline $\begin{array}{c}\text { General } \\
\text { absorbance case }\end{array}$ & Low & Low & Medium & Medium & High \\
\hline $\begin{array}{c}\mathrm{Pu}^{6+} \text { absorbance } \\
\text { case }\end{array}$ & Low & High & Low & High & --- \\
\hline Wavelength range & $425-860 \mathrm{~nm}$ & $450-700 \mathrm{~nm}$ & $520-850 \mathrm{~nm}$ & $520-700 \mathrm{~nm}$ & $\begin{array}{c}490-530 \& \\
560-620 \mathrm{~nm}\end{array}$ \\
\hline $\begin{array}{l}\text { Pre-processing } \\
\text { (X-block) }\end{array}$ & $\begin{array}{l}\text { SV(151,5,2) } \\
\text { Mean Center }\end{array}$ & $\begin{array}{l}\text { SV }(201,3,2) \\
\text { Mean Center }\end{array}$ & $\begin{array}{c}\text { SV }(201,3,2) \\
\text { Mean Center }\end{array}$ & $\begin{array}{l}\text { SV }(151,3,2) \\
\text { Mean Center }\end{array}$ & $\begin{array}{c}\text { SV(91,3,1) } \\
\text { Mean Center }\end{array}$ \\
\hline $\begin{array}{l}\text { Pre-processing } \\
\text { (Y-block) }\end{array}$ & \multicolumn{5}{|c|}{ Mean Center } \\
\hline Cross-validation & \multicolumn{5}{|c|}{$\begin{array}{c}8 \text { Random subsets } \\
3 \text { iterations }\end{array}$} \\
\hline \# PCs & 5 & 6 & 5 & 4 & 6 \\
\hline RMSEC (g/L) & 0.045 & 0.078 & 0.042 & 0.055 & 0.059 \\
\hline RMSECV (g/L) & 0.065 & 0.086 & 0.047 & 0.059 & 0.071 \\
\hline $\begin{array}{l}\text { Estimated signal- } \\
\text { to-noise ratio of } \\
\text { last PC }\end{array}$ & 3 & 8 & 6 & 55 & 30 \\
\hline $\begin{array}{l}\text { CV Correlation } \\
\text { coefficient (R2) }\end{array}$ & 0.9991 & 0.9985 & 0.9996 & 0.9993 & 0.9990 \\
\hline CV Bias (M) & 0.0005 & -0.0004 & 0.0004 & -0.0020 & 0.0011 \\
\hline $\begin{array}{l}\text { Limit of detection } \\
\text { (g/L) } \\
\text { (for 1" flow cell) }\end{array}$ & 0.02 & --- & --- & --- & --- \\
\hline
\end{tabular}

- The root mean-square errors in the tables are given as absolute values, which is a function of the modeling software output. In fact, as suggested by the validation plots, the plutonium prediction errors are more accurately represented as a percentage of the reading, not as a constant value. Table 4-5 shows the $1 \sigma$ uncertainties for each of the models. The values are shown are the sum of the absolute value of the bias and the standard deviation of the self-prediction, combined in quadrature with the uncertainty of the standards $(0.54 \%$, see Section 4.3$)$. These values will be used in determining the instrument uncertainty, as is discussed in Section 5.0.

- The model uncertainties roughly reflect the complexity of each model. The high acid solutions have the least amount of spectral variation, with only two $\mathrm{Pu}$ nitrate species, temperature variation, and a small amount of instrument variation accounting for the 4 PCs. The medium acid cases have a slightly greater uncertainty with the potential for three Pu nitrate species being present. The low acid cases have the greatest uncertainty, since multiple oxidation states arising from disproportionation must be included.

- As for the acid classification models, the high absorbance total Pu models require the use of first derivative preprocessing to obtain the best fitting results.

- Limits of detection, scaled for the 1" flow cell, are given for each low absorbance model. Recall that the analysis scheme will have the acidity classification model precede the total Pu model. For the blanks and very low plutonium concentration solutions, the absorbance signal is low and there is a larger variation in acidity reading. Therefore, in determining a limit of detection for the spectrophotometer as whole, the largest limit of detection must be cited, i.e. $0.02 \mathrm{~g} / \mathrm{L}$ for the lowacid model. 
SRNL-STI-2015-00454

Revision 0
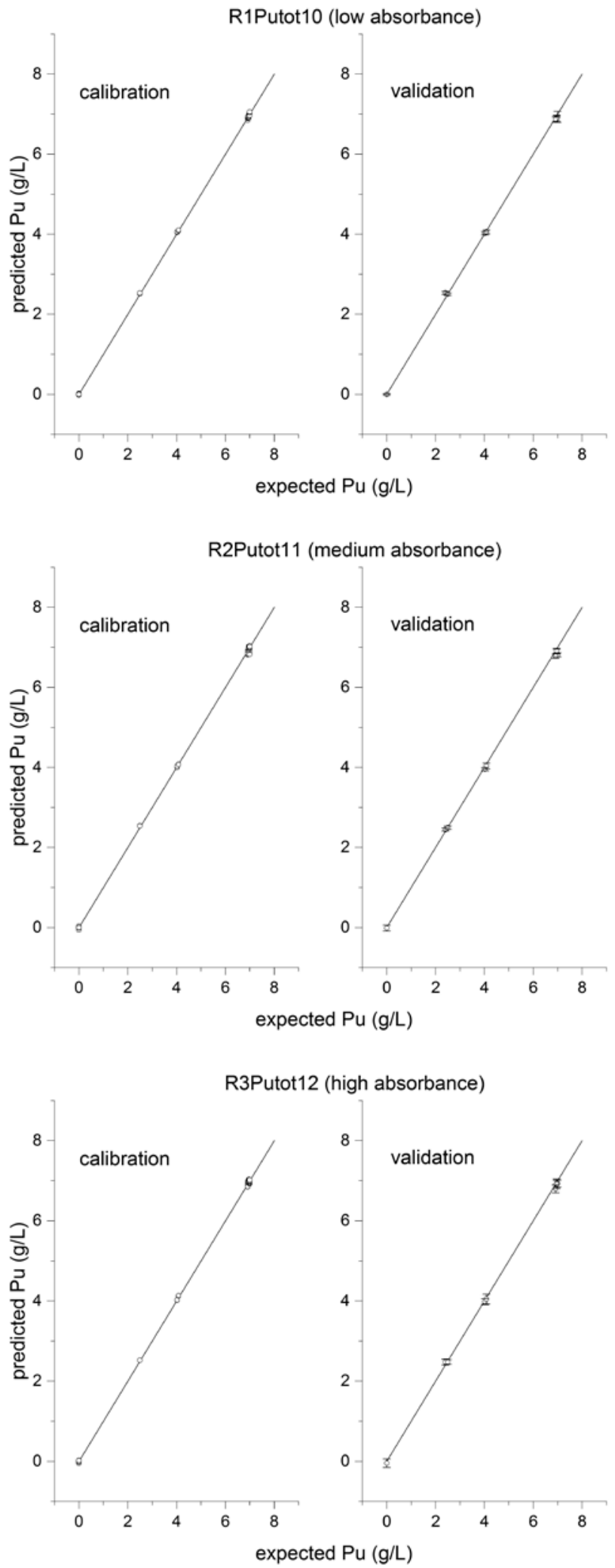

Figure 4-6. Residual plots for high acid total plutonium models. 
SRNL-STI-2015-00454

Revision 0
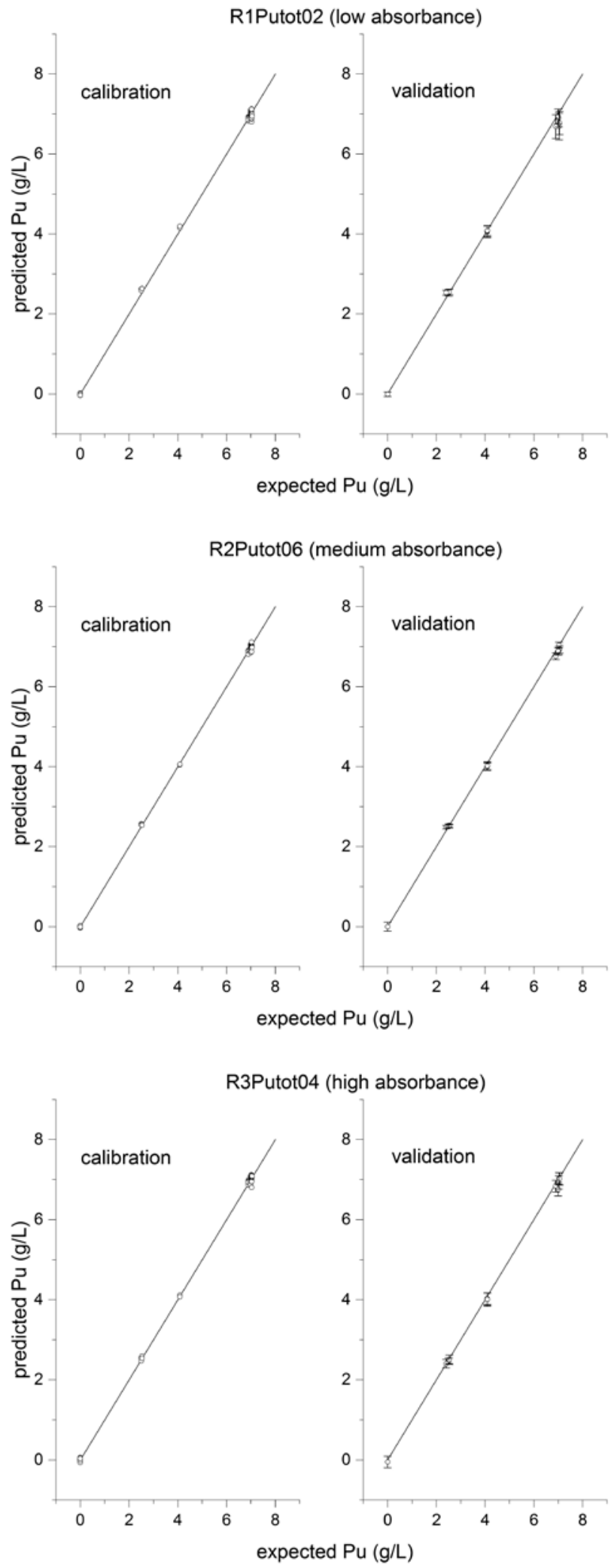

Figure 4-7. Residual plots for medium acid total plutonium models. 

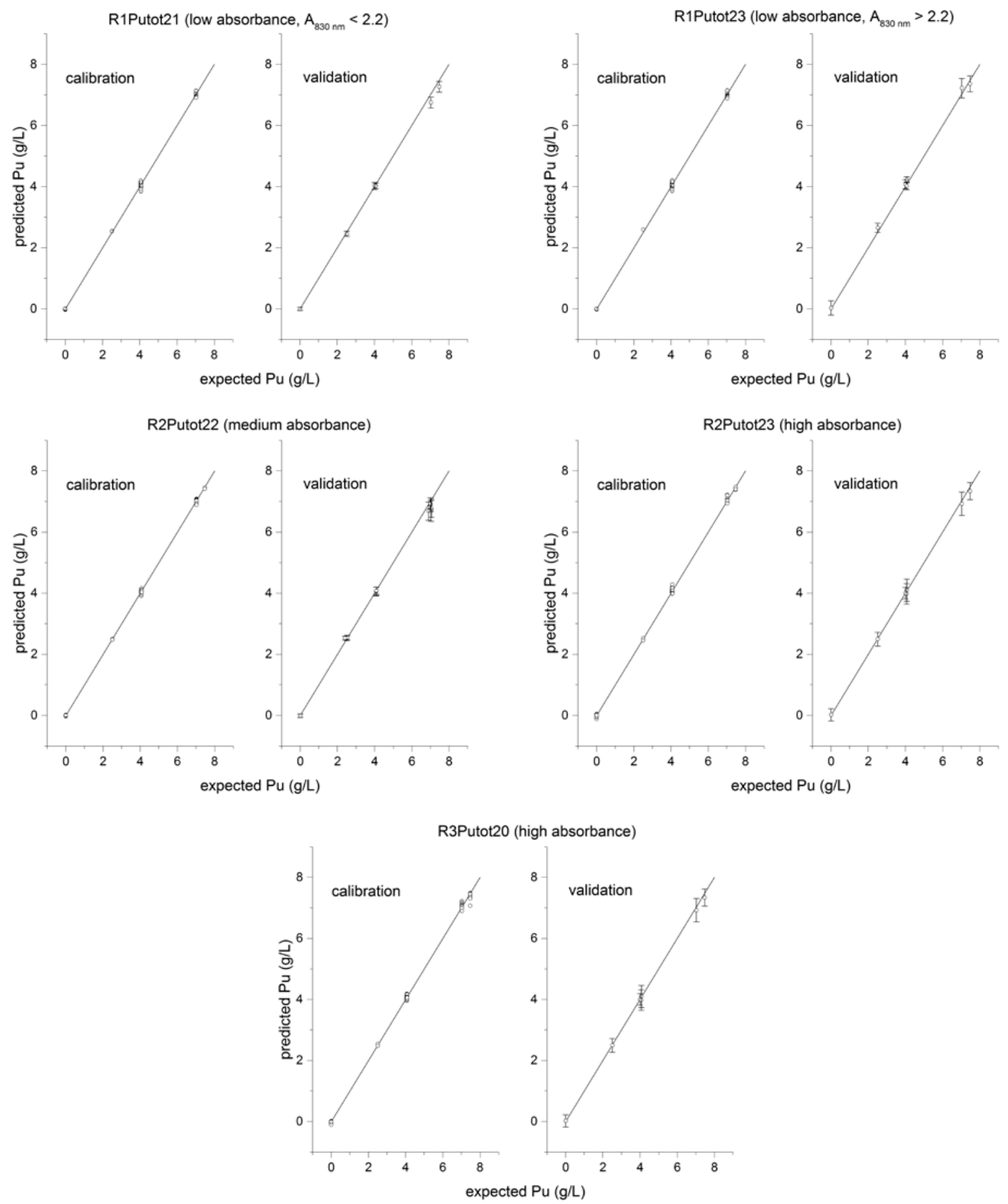

Figure 4-8. Residual plots for low acid total plutonium models. 


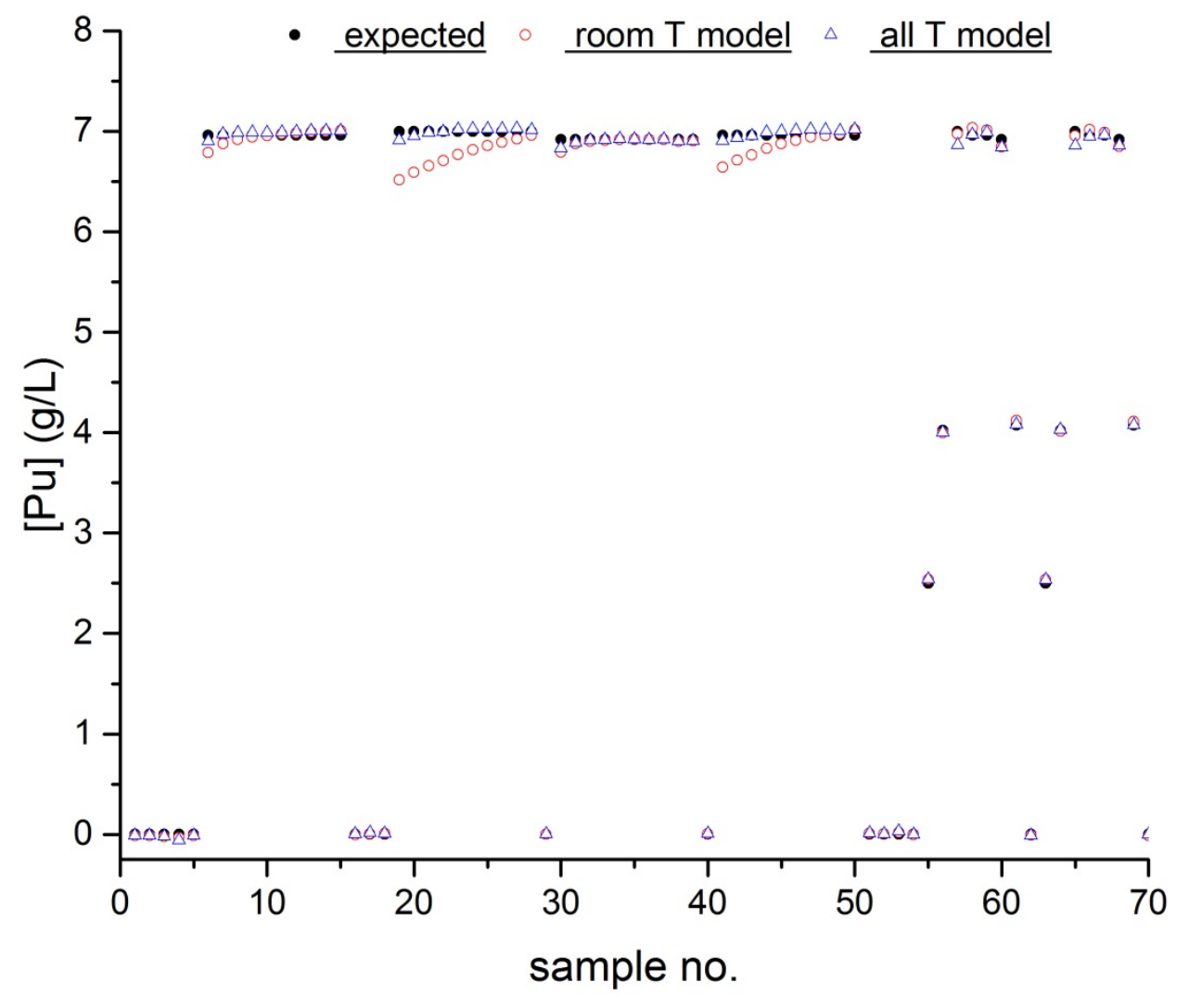

Figure 4-9. Temperature dependence of total Pu model R2Putot11.

Table 4-5. Uncertainties for total Pu models.

\begin{tabular}{|c|c|c|c|c|c|}
\hline & & \multicolumn{4}{|c|}{ Acid range } \\
\hline & & \multicolumn{2}{|c|}{ Low $(0.3-2 \mathrm{M})$} & \multirow{2}{*}{$\begin{array}{l}\text { Medium } \\
(2-6 \mathrm{M}) \\
\end{array}$} & \multirow{2}{*}{$\begin{array}{c}\text { High } \\
(6-9 \mathrm{M}) \\
\end{array}$} \\
\hline & & (1) $\mathrm{A}_{(830 \mathrm{~nm})}<2.2$ & (2) $A_{(830 \mathrm{~nm})}>2.2$ & & \\
\hline \multirow{3}{*}{ Absorbance } & Low & $\sigma=1.38 \%$ & $\sigma=2.07 \%$ & $\sigma=1.28 \%$ & $\sigma=0.78 \%$ \\
\hline & Medium & $\sigma=1.13 \%$ & $\sigma=1.77 \%$ & $\sigma=0.96 \%$ & $\sigma=0.91 \%$ \\
\hline & High & \multicolumn{2}{|c|}{$\sigma=1.49 \%$} & $\sigma=1.75 \%$ & $\sigma=0.70 \%$ \\
\hline
\end{tabular}


- Figure 4-10 indicates the plutonium concentration where the $\mathrm{A}=2.2$ threshold is met for each absorbance region. The changes for acidities greater than $6 \mathrm{M}$ come from the spectral shape changes associated with the higher-nitrated species. The rise at the lowest acidities comes from an apparent suppression of the molar absorptivity for $\mathrm{Pu}^{4+}$. The upper limit for the total $\mathrm{Pu}$ concentration that the instrument can read is given by the lowest point of the green curve, i.e. 13 g/L for medium acidities. Note that this value assumes a 1" flow cell.

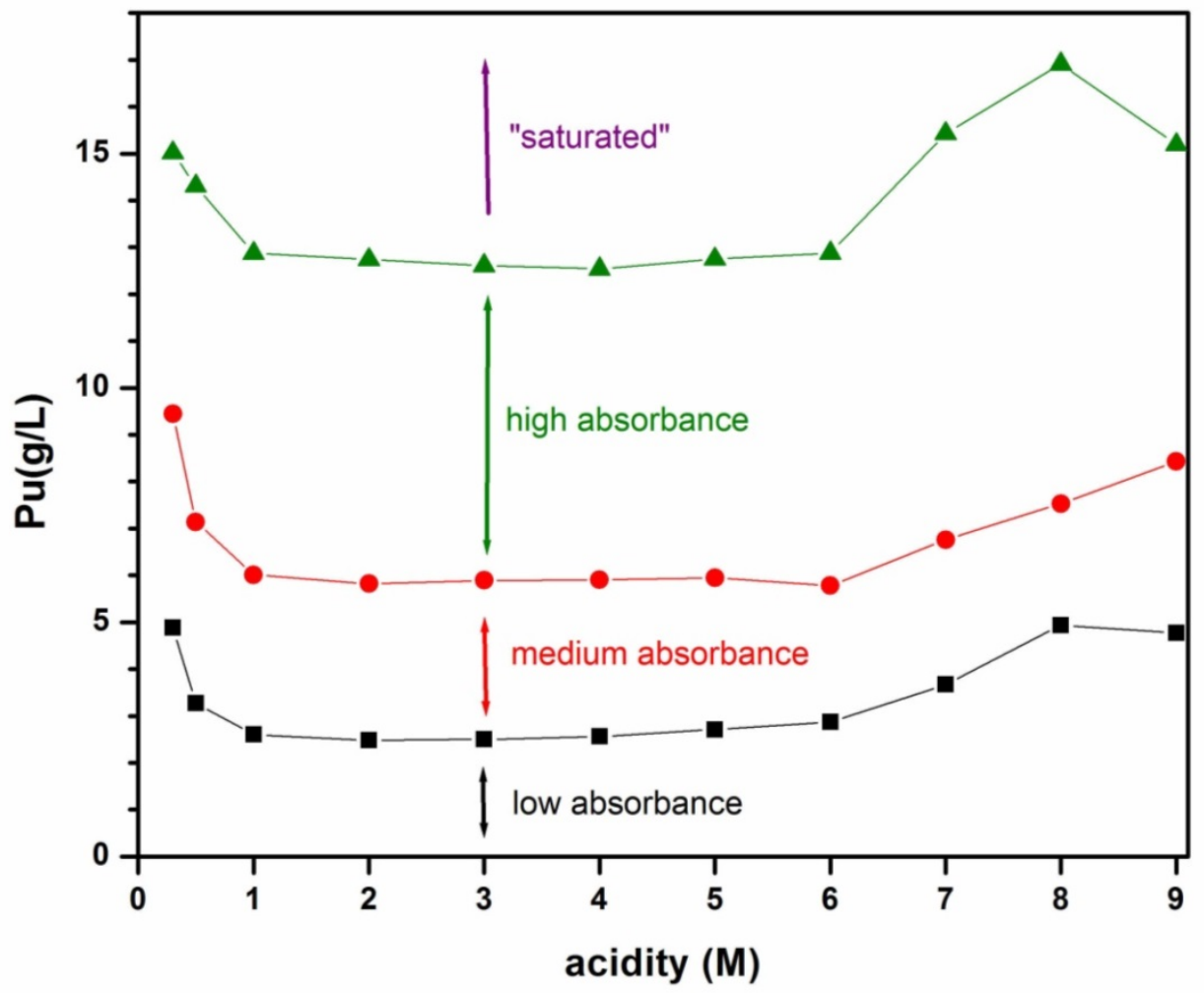

Figure 4-10. Relation of [Pu] to absorbance region for a 1 inch flow cell.

- The overall dynamic range of the spectrophotometer for total plutonium is the proportion of the maximum measurable value, $13 \mathrm{~g} / \mathrm{L}$, to the limit of detection, $0.02 \mathrm{~g} / \mathrm{L}$, or $650 \mathrm{x}$. This range is a factor of 2-3x larger than is typically obtainable with an absorption spectrometer. The improvements arise in part from the care taken in correcting for intensity response nonlinearities, and in part from the ability to apply different prediction models as the strongest peaks become saturated. The specific values for the lower and upper bounds will be dependent on the path length of the flow cell, but both will change proportionally with the path length. Thus, for a cell with a 0.1 ” path length, the range would become $0.2-130 \mathrm{~g} / \mathrm{L}$.

- The model validation data is also a measure of the inter-instrument variability. The calibrations generated with one certified instrument will be used directly on spectra obtained with other certified instruments. The variation observed within the certification tolerance must be included in the overall uncertainty analysis. The apparent inter-instrument variability is shown in Table 4-6. The uncertainties are roughly the same size as the self-prediction errors for the individual models, as shown in Table 4-5. The consistency of performance across spectrophotometers supports the notion that the spectrometers are essentially equivalent. However, this variability is real and must be accounted when determining the overall uncertainty for the spectrophotometers. 
Table 4-6. Inter-instrument variability as a function of total Pu calibration model.

\begin{tabular}{|c|c|c|c|c|c|}
\hline & & \multicolumn{4}{|c|}{ Acid range } \\
\hline & & \multicolumn{2}{|c|}{ Low $(0.3-2 \mathrm{M})$} & \multirow{2}{*}{$\begin{array}{l}\text { Medium } \\
(2-6 \mathrm{M}) \\
\end{array}$} & \multirow{2}{*}{$\begin{array}{c}\text { High } \\
(6-9 \mathrm{M}) \\
\end{array}$} \\
\hline & & (1) $\mathrm{A}_{(830 \mathrm{~nm})}<2.2$ & (2) $A_{(830 \mathrm{~nm})}>2.2$ & & \\
\hline \multirow{3}{*}{ Absorbance } & Low & $\sigma=1.33 \%$ & $\sigma=1.90 \%$ & $\sigma=1.84 \%$ & $\sigma=0.63 \%$ \\
\hline & Medium & $\sigma=1.78 \%$ & $\sigma=3.37 \%$ & $\sigma=0.85 \%$ & $\sigma=0.61 \%$ \\
\hline & High & \multicolumn{2}{|c|}{$\sigma=1.27 \%$} & $\sigma=1.59 \%$ & $\sigma=1.00 \%$ \\
\hline
\end{tabular}

\subsection{Pu calibration - Pu oxidation state distribution.}

Disproportionation of plutonium from the $4+$ to the $3+$ and $6+$ valence states will only occur at nitric acid concentrations below $2 \mathrm{M}$. The rate and final equilibrium of disproportionation will increase with decreasing nitric acid, being barely perceptible at $2 \mathrm{M}$ but rapid and very significant at $0.5 \mathrm{M}$ and below. Disproportionation also becomes more substantial as the plutonium concentration increases. [23-24] The rates are such that within the sealed cuvettes, the disproportionation of the low acidity standards can be observed in real time. Therefore, it was straightforward to generate a calibration set of spectra for $\mathrm{Pu}$ oxidation state models which contained a range of $3+$ and $6+$ concentrations as well as $4+$ concentrations.

Because the solutions were evolving as they were being analyzed, it was not possible to extract aliquots of the solutions to independently determine the concentrations of the individual oxidation states. However, there were constraints on the solutions and spectra that made possible an estimate of the concentrations. It was assumed that for these standards, there were no side reactions that altered the relative proportions of the three oxidation states. Thus, $3 \mathrm{Pu}^{4+}$ atoms would yield $2 \mathrm{Pu}^{3+}$ plus $1 \mathrm{Pu}^{6+}$ atoms, and there would always be twice as much $\mathrm{Pu}^{3+}$ as $\mathrm{Pu}^{6+}$. No heated standards were used in the calibration, as heating was found to promote the reoxidation of $\mathrm{Pu}^{3+}$ to $\mathrm{Pu}^{4+}$ (especially for samples between 1 to $2 \mathrm{M}$ nitric acid). The assumption was verified by principal component analysis (PCA), which indicated that all of the spectral variation during the evolution of a standard could be represented by two components. One component would be due to $\mathrm{Pu}^{4+}$ and the other to the combination of $\mathrm{Pu}^{3+}$ and $\mathrm{Pu}^{6+}$.

While there is overlap in the absorbance spectra for the different oxidation states, there are features that are attributable mainly to $\mathrm{Pu}^{6+}(\sim 830 \mathrm{~nm})$ and $\mathrm{Pu}^{4+}(\sim 475 \mathrm{~nm})$. Thus "pure" $\mathrm{Pu}^{4+}$ and $\mathrm{Pu}^{6+}$ components could be formed from linear combinations of the PCA components to best match those spectral features. Because the PCA components are orthogonal, and the $\mathrm{Pu}^{4+}$ and $\mathrm{Pu}^{6+}$ components are also constrained to be orthogonal, matching one feature would define both components. Specifically, the $\mathrm{Pu}^{4+}$ component is chosen to have minimal intensity near the $830 \mathrm{~nm} \mathrm{Pu}^{6+}$ peak.

This PCA analysis and feature rotation was performed individually for each low-acidity standard. When the predicted "pure" $\mathrm{Pu}^{6+}$ components were compared for standards ranging from 0.3 to $1 \mathrm{M}$, it was observed that the components were nearly identical. Over this range, the nitrato complex equilibrium does not change for $\mathrm{Pu}^{6+}$. All of the standards were then fit with the single pure $\mathrm{Pu}^{6+}$ component to estimate the $\mathrm{Pu}^{6+}$ concentrations. $\mathrm{Pu}^{3+}$ was defined as twice that of $\mathrm{Pu}^{6+}$, and the remainder of the plutonium was assumed to be $\mathrm{Pu}^{4+}$.

Once the oxidation state distribution was determined for each of the standard spectra, PLS prediction models for $\mathrm{Pu}^{3+}$ and $\mathrm{Pu}^{6+}$ were generated in the same manner as for the total Pu models. The preferred approach for measuring disproportionation is to use the $\mathrm{Pu}^{6+}$ peak, which is strong and has less overlap with $\mathrm{Pu}^{4+}$ absorbance. The absorbance check at $830 \mathrm{~nm}$ confirms that this peak is on scale. If so, the $\mathrm{Pu}^{6+}$ model is applied, and the result is used to calculate $\mathrm{Pu}^{3+}$ and $\mathrm{Pu}^{6+}$. If the $830 \mathrm{~nm}$ peak is off-scale, the $\mathrm{Pu}^{3+}$ model is used instead. The parameters for each model are given in Table 4-7. 
Table 4-7. Pu oxidation state model parameters.

\begin{tabular}{|c|c|c||}
\hline Model name & PLS_Pu6b_ & PLS_Pu3c \\
\hline Absorbance case & Low (A(830 $\mathrm{nm})<2.2)$ & High $(\mathrm{A}(830 \mathrm{~nm})>2.2)$ \\
\hline Wavelength range & 800-850 nm & $520-650 \mathrm{~nm}$ \\
\hline Pre-processing (X-block) & $\begin{array}{c}\text { SG(93,3,2) } \\
\text { Mean Center }\end{array}$ & $\begin{array}{c}\text { SG(93,3,2) } \\
\text { Mean Center }\end{array}$ \\
\hline Pre-processing (Y-block) & Mean Center & Mean Center \\
\hline Cross-validation & $\begin{array}{c}\text { 8 Random subsets } \\
\text { 3 iterations }\end{array}$ & $\begin{array}{c}\text { 8 Random subsets } \\
\text { 3 iterations }\end{array}$ \\
\hline \# PCs & 1 & 3 \\
\hline RMSEC (g/L) & 0.00035 & 0.019 \\
\hline RMSECV (g/L) & 0.00036 & 0.020 \\
\hline $\begin{array}{c}\text { CV Correlation coefficient } \\
\text { (R2) }\end{array}$ & 0.999995 & -0.0002 \\
\hline CV Bias (M) & 0 & -- \\
\hline $\begin{array}{c}\text { Limit of detection (g/L) } \\
\text { (for 1” flow cell) }\end{array}$ & 0.01 & \\
\hline \hline
\end{tabular}

- Neither model uses the 460-510 nm region that is the subject of the first absorbance check. Each model applies equally to the low and medium absorbance cases.

- The excellent fit of the $\mathrm{Pu}^{6+}$ results with a single principal component is further confirmation of the consistency of the peak across this limited nitric acid range and of the lack of spectral interference from $\mathrm{Pu}^{4+}$. In contrast, there is substantial overlap between the $\mathrm{Pu}^{3+}$ and $\mathrm{Pu}^{6+}$ peaks, which require extra principal components to resolve.

- Because elevated temperature changes the disproportionation equilibrium ratio and there was no way to independently determine the oxidation state proportions, it was not possible to determine the effect of temperature on the accuracy these predictions. At this time the oxidation state distribution information is informational, rather than necessary for process control or accountability. However, the $\mathrm{Pu}^{6+}$ peak is very strong and thus it is likely that small quantities of that oxidation state will be indicated. 


\subsection{Uncertainty analysis}

The uncertainty analysis for the spectrophotometers, with specific comparison to the colorimeters, has been issued as an internal memorandum. ${ }^{\text {a }}$ Several contributing factors to the analysis, such as calibration model uncertainty and inter-instrument variability, have been discussed above. The remaining aspects of the calculation are presented here. The full uncertainty analysis, including DCS loop uncertainties, is found in Ref. [24].

Table 5-1 lists the input data and values for the spectrophotometer. Each entry is discussed in turn.

Table 5-1. Inputs for uncertainty calculations.

\begin{tabular}{|c|c|}
\hline Input Data & Value \\
\hline 1. Instrument range & $0-13 \mathrm{~g} / \mathrm{L} \mathrm{Pu}$ (absorbance $=0-2.2$ ) \\
\hline 2. $\quad$ Calibration model uncertainty & Varies by specific calibration \\
\hline 3. $250 \Omega$ input resistor tolerance & $\pm 0.03 \%$ \\
\hline 4. Analog In module resolution & 14 bit \\
\hline 5. Analog In module absolute accuracy error @25C & Max $\pm 0.05 \%$ full scale \\
\hline 6. Analog In module linearity & $\pm 0.008 \%$ \\
\hline 7. Maximum zero shift & $0.0036 \mathrm{~g} / \mathrm{L} \mathrm{Pu}$ \\
\hline 8. Long term output drift & $0.9 \%$ \\
\hline 9. Analog In module accuracy drift with temperature & $\operatorname{Max} \pm 0.004 \%$ full scale $/{ }^{\circ} \mathrm{C}$ \\
\hline 10. Full scale (1” flow cell) & $\sim 13 \mathrm{~g} / \mathrm{L} \mathrm{Pu}$ \\
\hline 11. Inter-instrument variability & Varies by specific calibration \\
\hline 12. Path length & $0.60 \%$ \\
\hline 13. Acid classification error & $0-4.01 \%$ (varies by specific calibration) \\
\hline
\end{tabular}

1, 10. Instrument range and Full scale. Figure 4-10 defines the upper end of the instrument range as 13 $\mathrm{g} / \mathrm{L}$ for a 1" flow cell. The lower end of the range is nominally $0 \mathrm{~g} / \mathrm{L}$, but practically is defined by the limit of detection (LOD), below which the reading is statistically indistinguishable from zero. For the three low absorbance models, these values are 0.021 (low acidity), 0.019 (medium acidity), and 0.010 (high acidity) g/L.

2. Calibration model uncertainty. These values are specific to the individual models and are given in Table 4-5. The values include the self-prediction error for the model, including all variation due to acidity, temperature, and (where appropriate) disproportionation, and the uncertainty of the Pu concentrations in the standards. Note that these calibrations eliminate the uncertainty source terms due to low acid bias and disproportionation that are significant in the uncertainty calculation for the colorimeters.

3-6, 9. $250 \Omega$ input resistor tolerance, Analog In module resolution, Analog In module absolute accuracy error@25C, Analog In module linearity, and Analog In module accuracy drift with temperature. These values are related to the DCS, and remain unchanged from the colorimeter calculation.

7. Maximum zero shift. This quantity is expected to be the same at all parts of the instrument scale. The maximum zero shift was determined by repeated measurement of a very low concentration Pu solution over 80 hours. The solution was the remnant after a column simulation run, and was approximately 0.7 $\mathrm{g} / \mathrm{L}$ in low acid. It was held in a $1 \mathrm{~mm}$ flow cell, so that the equivalent concentration for a 1 inch flow cell would be $\sim 0.7 / 25.4=0.028 \mathrm{~g} / \mathrm{L}$. The overall standard deviation $(1 \sigma)$ for this set is $0.0036 \mathrm{~g} / \mathrm{L}$. The time dependence of the data is shown in Figure 5-1. There is a slight overall downward trend to the data,

${ }^{a}$ R.J. Lascola to D. Stimac and C.R. Loyal, “Uncertainty Analysis for AFS-2 Spectrophotometers”, SRNL-L4000-2015-00029, July 29, 2015. 
indicated by the regression line, with an apparent rate of change of $\sim-8 \times 10^{-5} \mathrm{~g} / \mathrm{L} / \mathrm{hr}$, although the trend is much smaller than the spread of data (as indicated by the low regression value $r^{2}=0.26$ ). Analysis of the data up to $t=25$ hours shows no statistically significant drift. Information about the changes in laboratory conditions during this test is not available. The self-referencing design of the spectrophotometer explicitly measures and corrects for drifts in lamp color and intensity, and the data processing in the total Pu calibrations corrects for other effects associated with the fibers and flow cell. Therefore, the standard deviation observed for the data set is a more realistic measure of the variability of the blank / low level measurement.

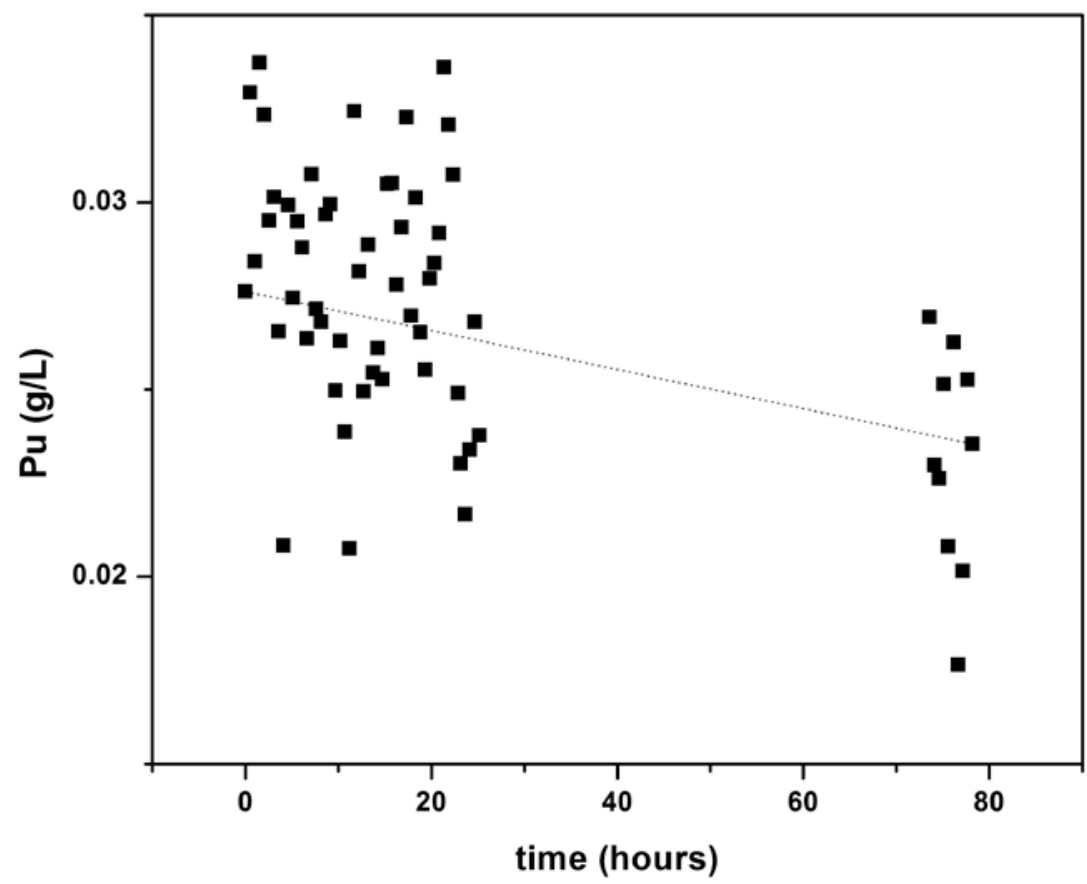

Figure 5-1. Zero drift observations.

8. Long term output drift. This quantity is a proportional value. Long term output drift was determined by measuring the concentration of a $\sim 7 \mathrm{~g} / \mathrm{L} \mathrm{Pu}, 1 \mathrm{M}$ solution over 5.8 days. The results of the measurement are shown in Figure 5-2. The standard deviation $(1 \sigma)$ of the measurement over this time is $0.062 \mathrm{~g} / \mathrm{L}$, or $0.9 \%$ of the mean measurement.

It should be noted that the laboratory conditions within this testing period started with a steam outage, with the laboratory temperature $<20 \mathrm{C}$. The steam outage ended at $\sim 15-18$ hours, with rapid heating of the lab by at least 10-15 C, followed by a slow cooling over the remainder of the test period. The instrument reading tracked with temperature. The drift of the instrument under consistent environmental conditions will likely be less than the overall drift observed here. There was also a slight amount of condensation that appeared in the head space of the sealed cuvette, which would increase the concentration of the solution. This effect is beyond what would be expected for the process. Therefore, this study encompasses and exceeds the variation that should be observed in the field. Note that the reported value is not a linear drift per unit time.

11. Inter-instrument variability. This information is provided in Table 4-6. 


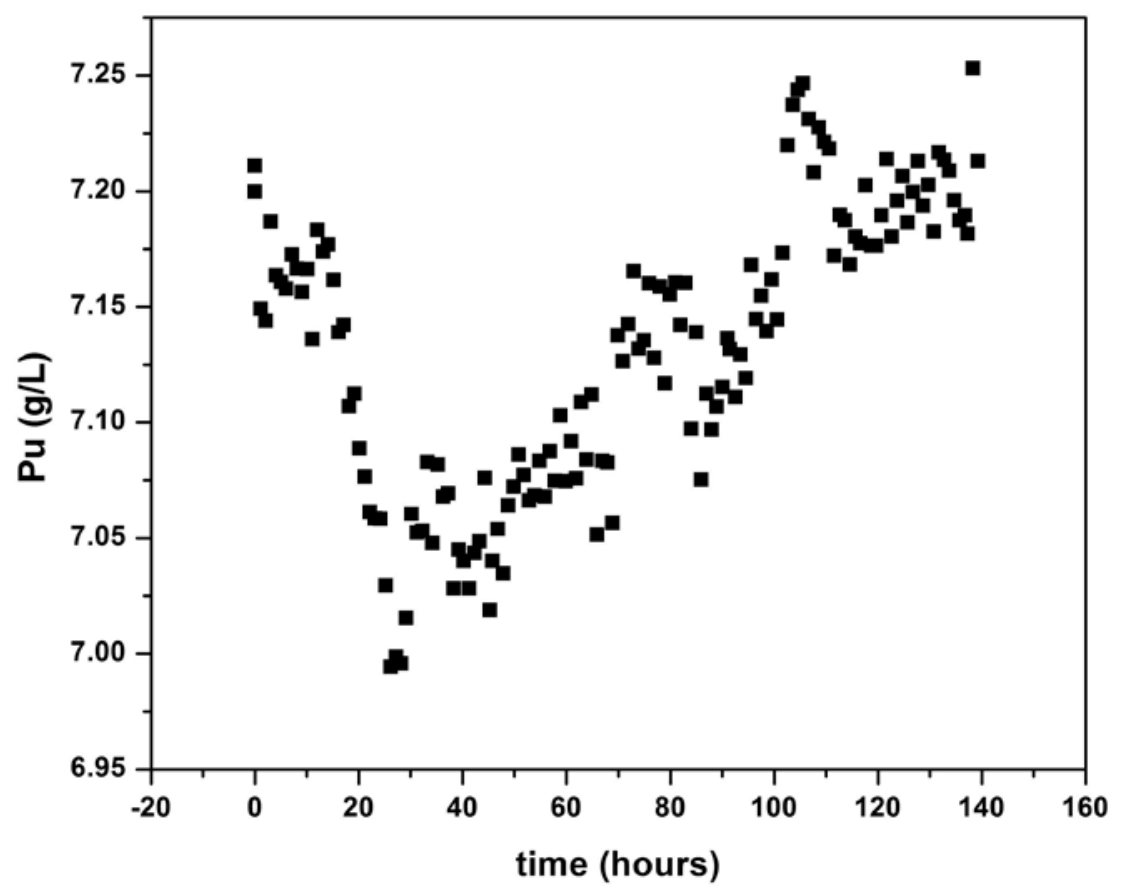

Figure 5-2. Measurement of long-term drift.

12. Path length. The $1 \mathrm{~cm}$ quartz cuvettes used to hold the Pu calibration standards have a pathlength tolerance of $\pm 0.01 \mathrm{~mm}$, or $0.1 \%$. The measurement of the pathlength of the flow cell has a tolerance of $0.59 \%$. [25] When combined in quadrature, these quantities yield a total pathlength uncertainty of $\left.\left[(0.1 \%)^{2}+(0.59 \%)^{2}\right)\right]^{1 / 2}=0.60 \%$. Note that the tolerance for the cuvettes is less than the $1.0 \%$ cited for different cuvettes that were used in colorimeter calibrations.

13. Effects of acidity misclassification. The potential for an error in total plutonium reading due to misclassification of solution acidity has been described above (Section 4.4). Assessing this error first requires identifying which solutions are likely to be misclassified. Then, it must be determined if the measurement error is likely to be significantly greater for that solution than the expected error if the solution were within the calibration range.

The true acid classification uncertainty includes both the model uncertainty and the uncertainty of the acidity of the standards. The former is discussed in Section 4.4. The latter varies from $7.0-8.7 \%(1 \sigma)$, based on the titration method used to characterize the standards. For simplicity and to be conservative, the largest value is assumed for all standards. These values are added in quadrature to provide a total classification uncertainty.

The overall process for assessing classification error is best illustrated with a specific example. For cases of medium absorbance, the acid classification model has an uncertainty of $0.18 \mathrm{M}(1 \sigma)$. At $6 \mathrm{M}$, the standard uncertainty is $6 \times 0.087=0.52 \mathrm{M}$. In quadrature, these values combine to $0.55 \mathrm{M}$. For the high acidity model, which is applicable to solutions from 6-9 $\mathrm{M}$, the case of a $6-0.55=5.45 \mathrm{M}$ solution must be considered. While a standard with this acidity was not measured, a $5 \mathrm{M}$ solution was measured. This solution was found to measure $(-5.21) \% \pm 0.57 \%(1 \sigma)$, compared to a mean error for the 6-9 M solutions of $(-0.83) \% \pm 0.71 \%(1 \sigma)$ (this data includes measurements from the inter-instrument variability set). Therefore, there is a difference of $(-4.38) \% / \mathrm{M} \pm 1.01 \%(1 \sigma)$. For a difference of $0.55 \mathrm{M}$, then, a measurement difference of $-2.4 \% \pm 0.6 \%(1 \sigma)$ below the mean is expected. This difference exceeds, 
within $1 \sigma$ bounds, the 6-9 $\mathrm{M}$ mean $(|-2.4 \%|>(0.71 \%+0.6 \%))$. Therefore, for this example, the effect of acidity misclassification produces a statistically significant result.

Applying this calculation to all 11 total Pu models, there are three cases for which the anticipated error is statistically significant. One case is in the above example (medium absorbance/high acid). For the medium absorbance/medium acid case, solutions at $1.82 \mathrm{M}$ (the low acid end) may read $1.40 \%$ above the mean value from 2-6 M, compared to a combined $1 \sigma$ uncertainty of $0.81 \%$. (Measurement error for solutions at the high acidity end of the model range $(6.18 \mathrm{M})$ is not distinguishable from solutions within the calibration range.) For the high absorbance/medium acid model, solutions on the high acid end may read $-4.01 \%$ below the mean value from 2-6 M, compared to a combined $1 \sigma$ uncertainty of $1.73 \%$. These errors will be included as they apply to specific processing scenarios.

Combination of uncertainties. The overall uncertainty for the spectrophotometers will include contributions from the instruments and from the DCS. The latter contributions will not be discussed here. Spectrophotometer instrument uncertainties can be put into three groups and combined accordingly:

- $\quad$ Overall calibration uncertainty. These are proportional uncertainties associated with laboratory calibration, and include calibration model uncertainty $\left(\sigma_{2}\right)$, inter-instrument variability $\left(\sigma_{11}\right)$, pathlength correction uncertainty $\left(\sigma_{12}\right)$, and acid classification uncertainty $\left(\sigma_{13}\right)$. As noted in the discussion, $\sigma_{2}, \sigma_{11}$, and $\sigma_{13}$ will depend on the solution absorbance and acidity.

- Long term output drift. This value $\left(\sigma_{8}\right)$ is a proportional value.

- Maximum zero drift. This value $\left(\sigma_{7}\right)$ is an absolute value.

The instrument uncertainty, in $\mathrm{g} / \mathrm{L}$, for an instrument reading $R$ is given by the following formula:

$$
\sigma_{R}=\left[\sigma_{7}^{2}+\left(R \sigma_{8}\right)^{2}+\left(R\left(\sigma_{2}^{2}+\sigma_{11}^{2}+\sigma_{12}^{2}+\sigma_{13}^{2}\right)^{1 / 2}\right)^{2}\right]^{1 / 2}
$$

For reference, Table 5-2 gives the percentage error expected for the various solution conditions. $R$ is set to $2 \mathrm{~g} / \mathrm{L}$ for the low absorbance case, $6 \mathrm{~g} / \mathrm{L}$ for the medium absorbance case, and $13 \mathrm{~g} / \mathrm{L}$ for the high absorbance case (assuming a 1" flow cell). For the medium acid cases where acid misclassification at one edge of the acidity range has significance, the additional uncertainty (and applicable range) is noted.

Table 5-2. Total uncertainties for various process conditions.

\begin{tabular}{|c|c|c|c|c|c|}
\hline & \multicolumn{4}{|c|}{ Acid range } \\
\hline & & \multicolumn{2}{|c|}{ Low $(0.3-2 \mathrm{M})$} & \multirow{2}{*}{$\begin{array}{l}\text { Medium } \\
(2-6 \mathrm{M}) \\
\end{array}$} & \multirow{2}{*}{$\begin{array}{c}\text { High } \\
(6-9 \mathrm{M}) \\
\end{array}$} \\
\hline & & (1) $A_{(830 \mathrm{~nm})}<2.2$ & (2) $A_{(830 \mathrm{~nm})}>2.2$ & & \\
\hline \multirow{3}{*}{ Abs. } & Low & $\sigma=2.21 \%$ & $\sigma=3.02 \%$ & $\sigma=2.50 \%$ & $\sigma=1.49 \%$ \\
\hline & Medium & $\sigma=2.37 \%$ & $\sigma=3.96 \%$ & $\begin{array}{l}\sigma=2.22 \%(<3 \mathrm{M}) \\
\sigma=1.73 \%(3-6 \mathrm{M})\end{array}$ & $\sigma=2.85 \%$ \\
\hline & High & \multicolumn{2}{|c|}{$\sigma=2.24 \%$} & $\begin{array}{c}\sigma=2.60 \%(2-5 \mathrm{M}) \\
\sigma=4.78 \%(>5 \mathrm{M})\end{array}$ & $\sigma=1.63 \%$ \\
\hline
\end{tabular}

Applicability to process conditions. Table 5-3 provides the expected conditions (Pu concentration, acidity) for the steps in the AFS-2 process where the spectrophotometers are expected to perform safety and/or process control functions. The different process conditions imply that different calibrations, and thus different uncertainties, may apply at each stage. Where several calibration regions are indicated, the table lists the highest uncertainty associated with those regions. 
Table 5-3. Association of uncertainties with process steps.

\begin{tabular}{|c|c|c|c|c|c|}
\hline $\begin{array}{l}\text { On-Line } \\
\text { Monitor }\end{array}$ & $\begin{array}{l}\text { Process } \\
\text { Step }\end{array}$ & $\begin{array}{c}\text { Projected } \\
\text { Solution Conditions }\end{array}$ & $\begin{array}{l}\text { Calibration } \\
\text { Region }\end{array}$ & $\begin{array}{l}\text { Instrument } \\
\text { uncertainty } \\
(1 \sigma)^{*}\end{array}$ & Comments \\
\hline \multirow[t]{7}{*}{$\begin{array}{l}\text { NK-1 } \\
\text { /NK-3 }\end{array}$} & $\begin{array}{l}\text { Column } \\
\text { Recondition }\end{array}$ & $\begin{array}{l}\text { Pu: } 0 \mathrm{~g} / \mathrm{L} \\
\text { Acid: } 8 \mathrm{M}\end{array}$ & $\begin{array}{l}\text { Abs: low } \\
\text { Acid: high }\end{array}$ & $\begin{array}{l}2.06 \% @ 9 \\
0.25 \mathrm{~g} / \mathrm{L}\end{array}$ & $\mathrm{LOD}=0.10 \mathrm{~g} / \mathrm{L}$ \\
\hline & $\begin{array}{l}\text { Column } \\
\text { Adsorption }\end{array}$ & $\begin{array}{l}\text { Pu: } 0 \rightarrow 3.5 \mathrm{~g} / \mathrm{L} \\
\text { Acid: } 6-8 \mathrm{M}\end{array}$ & $\begin{array}{l}\text { Abs: medium } \\
\text { Acid: high }\end{array}$ & $\begin{array}{l}2.85 \% @ 9 \\
3.5 \mathrm{~g} / \mathrm{L}\end{array}$ & \\
\hline & $\begin{array}{l}\text { Column } \\
\text { Decon }\end{array}$ & $\begin{array}{l}\text { Pu: } 3.5 \rightarrow 0 \mathrm{~g} / \mathrm{L} \\
\text { Acid: } 8 \mathrm{M}\end{array}$ & $\begin{array}{l}\text { Abs: medium } \\
\text { Acid: high }\end{array}$ & $\begin{array}{l}2.85 \% @ 9 \\
3.5 \mathrm{~g} / \mathrm{L}\end{array}$ & \\
\hline & $\begin{array}{l}\text { Elution- } \\
\text { Head Cut }\end{array}$ & $\begin{array}{l}\text { Pu: } 0 \rightarrow>4 \mathrm{~g} / \mathrm{L} \\
\text { Acid: } 8-3 \mathrm{M} * \\
\text { Current cut pt: } 1 \mathrm{~g} / \mathrm{L} \\
\text { Desired cut pt: }>4 \mathrm{~g} / \mathrm{L}\end{array}$ & $\begin{array}{l}\text { Abs: med/high } \\
\text { Acid: med/high }\end{array}$ & $\begin{array}{l}\text { 4.78\% @ } 6 \\
\text { g/L }\end{array}$ & $\begin{array}{l}6 \mathrm{~g} / \mathrm{L} \text { case assumes high } \\
\text { absorbance, medium acid } \\
\text { (highest uncertainty of the } \\
\text { four possibilities) }\end{array}$ \\
\hline & $\begin{array}{l}\text { Elution- } \\
\text { Heart Cut }\end{array}$ & $\begin{array}{l}\text { Pu: }>100 \rightarrow>4 \mathrm{~g} / \mathrm{L} \\
\text { Acid: }<1 \mathrm{M} \\
\text { Current cut pt: } 1 \mathrm{~g} / \mathrm{L} \\
\text { Desired cut pt: }>4 \mathrm{~g} / \mathrm{L}\end{array}$ & $\begin{array}{l}\text { Abs: med/high } \\
\text { Acid: low(1) }\end{array}$ & $\begin{array}{l}2.37 \% @ 6 \\
g / L\end{array}$ & \\
\hline & $\begin{array}{l}\text { Elution- Tail } \\
\text { Cut }\end{array}$ & $\begin{array}{l}\text { Pu: }>4 \mathrm{~g} / \mathrm{L} \\
\text { Acid: }<1 \mathrm{M}\end{array}$ & $\begin{array}{l}\text { Abs: med/low } \\
\text { Acid: low(1) }\end{array}$ & $\begin{array}{l}2.77 \% \text { @ } \\
0.25 \mathrm{~g} / \mathrm{L} \\
\end{array}$ & $\begin{array}{l}\text { Transition from medium to } \\
\text { low abs. is } \sim 2-3 \mathrm{~g} / \mathrm{L} \text {. }\end{array}$ \\
\hline & $\begin{array}{l}\text { Extended } \\
\text { Elution For } \\
\text { Resin } \\
\text { Removal or } \\
\text { Instrument } \\
\text { Calibration }\end{array}$ & $\begin{array}{l}\text { Pu: }>4 \rightarrow 0 \mathrm{~g} / \mathrm{L} \\
\text { Acid: } 0.5-0.35 \mathrm{M}\end{array}$ & $\begin{array}{l}\text { Abs: med to } \\
\text { low } \\
\text { Acid: low(1) }\end{array}$ & $\begin{array}{l}2.37 \% @ 4 \\
\text { g/L } \\
2.77 \% @ \\
0.25 \mathrm{~g} / \mathrm{L}\end{array}$ & $\mathrm{LOD}=0.021 \mathrm{~g} / \mathrm{L}$ \\
\hline \multirow[t]{4}{*}{ Raffinate } & $\begin{array}{l}\text { Elution- } \\
\text { Head Cut to } \\
11.1\end{array}$ & $\begin{array}{l}\text { Pu: } 0 \rightarrow>4 \mathrm{~g} / \mathrm{L} \\
\text { Acid: } 8-3 \mathrm{M}^{* *} \\
\text { Current cut pt: } 1 \mathrm{~g} / \mathrm{L} \\
\text { Desired cut pt: }>4 \mathrm{~g} / \mathrm{L}\end{array}$ & $\begin{array}{l}\text { Abs: med/high } \\
\text { Acid: med/high }\end{array}$ & $\begin{array}{l}\text { 4.78\%@6 } \\
\text { g/L }\end{array}$ & $\begin{array}{l}6 \mathrm{~g} / \mathrm{L} \text { case assumes high } \\
\text { absorbance, medium acid } \\
\text { (highest uncertainty of the } \\
\text { four possibilities) }\end{array}$ \\
\hline & $\begin{array}{l}\text { Elution- Tail } \\
\text { Cut to } 11.1 \\
\end{array}$ & $\begin{array}{l}\text { Pu: }>4 \mathrm{~g} / \mathrm{L} \\
\text { Acid: }<1 \mathrm{M}\end{array}$ & $\begin{array}{l}\text { Abs: med } \\
\text { Acid: low(1) }\end{array}$ & $\begin{array}{l}2.37 \% @ 4 \\
\text { g/L }\end{array}$ & \\
\hline & $\begin{array}{l}\text { Extended } \\
\text { Elution For } \\
\text { Resin } \\
\text { Removal }\end{array}$ & $\begin{array}{l}\text { Pu: }>4 \rightarrow 0 \mathrm{~g} / \mathrm{L} \\
\text { Acid: } 0.5-0.35 \mathrm{M}\end{array}$ & $\begin{array}{l}\text { Abs: med to } \\
\text { low } \\
\text { Acid: low(1) }\end{array}$ & $\begin{array}{l}2.37 \% @ 4 \\
\text { g/L } \\
2.77 \% @ \\
0.25 \mathrm{~g} / \mathrm{L}\end{array}$ & $\mathrm{LOD}=0.021 \mathrm{~g} / \mathrm{L}$ \\
\hline & $\begin{array}{l}\text { NT-11/12 } \\
\text { Drop to H } \\
\text { (Column } \\
\text { Bypass) }\end{array}$ & $\begin{array}{l}\text { Pu: } 2.5->4 \mathrm{~g} / \mathrm{L} \\
\text { Acid: } 6 \text { - } 8 \mathrm{M}\end{array}$ & $\begin{array}{l}\text { Abs: med } \\
\text { Acid: high }\end{array}$ & $\begin{array}{l}\text { 2.85\% @ } 6 \\
\text { g/L }\end{array}$ & $\begin{array}{l}\text { 1.73\% @ } 6 \text { g/L for medium } \\
\text { absorbance, including high } \\
\text { acid side classification } \\
\text { error. }\end{array}$ \\
\hline
\end{tabular}

* Uncertainties do not include DCS loop uncertainty contributions.

** Per Figure 6-3, at the transition between the head and heart cut, the acidity will be in the "medium" range, e.g. between $2-6 \mathrm{M}$.

The low acid, track (2) is not considered because the solution conditions leading to that track $\left(\mathrm{A}_{830 \mathrm{~nm}}>\right.$ 2.2) are not credible for the process. Roughly, $\sim 0.4 \mathrm{~g} / \mathrm{L}$ of $\mathrm{Pu}^{6+}$ must be present for $\mathrm{A}_{830 \mathrm{~nm}}=2.2$ in a 1" 
flow cell, for which a total of $1.2 \mathrm{~g} / \mathrm{L} \mathrm{Pu}^{4+}$ must be lost through disproportionation. At $6 \mathrm{~g} / \mathrm{L}$ total Pu, this corresponds to a $20 \%$ disproportionation rate, which exceeds any observed or expected quantity.

\subsection{Qualifications}

Qualification tests were conducted to demonstrate that the spectrophotometers, in the form in which they will be delivered to the customer, are properly operational. The tests demonstrate not only the ability to provide accurate $\mathrm{Pu}$ measurements, but also the ability to produce expected alarm responses when the appropriate conditions are present.

The first test was a confirmation that the three assembled units can reproduce expected responses for plutonium calibration standard solutions in sealed cuvettes. These measurements demonstrate the ability to properly classify solutions into the expected categories of acidity and solution absorbance as well as the continued accuracy of measurements several months after the original calibration spectra were obtained for the solutions. A comparison of the performance of the three instruments is given in Figure 6-1. The instruments are identified by the NGS labels as described above. The standard solutions were contained in $1 \mathrm{~cm}$ cuvettes, which reduced the observed absorbances compared to those expected for the 1 inch flow cell. Thus, these standards are not expected to trigger the "high absorbance" case, and only the highest concentrations ( $\sim 7 \mathrm{~g} / \mathrm{L})$ might trigger the "medium absorbance" case. The absorbance spectra were manually evaluated to confirm that the program selected the proper absorbance case. All acid classifications were correctly identified, with $2 \mathrm{M}$ solutions distributed between low and medium acidity and $6 \mathrm{M}$ solutions tending towards medium acidity. The spectra of solutions identified as "low acid" were also manually checked and confirmed to accurately classify absorbances at $830 \mathrm{~nm}$ (all Track (1), low $\left.\mathrm{Pu}^{6+}\right)$.

Prediction accuracy, as shown in the top panel of Figure 6-1, is within $\pm 3 \%$ for all three units, which is within the $2 \sigma$ uncertainty value. Measurement errors track well between instruments. There is almost correlation between measurement error and either plutonium $(\rho=-0.09)$ or acid $(\rho=-0.13)$ concentration. For the four solutions with either 2 and $6 \mathrm{M}$ acidity, the mean difference between the two possible models was $\sim 3.5 \%$. These results indicate that the instruments are performing within specifications.

The second test was the use of one of the spectrophotometers to monitor the output of two column elutions conducted in the laboratory. This test establishes the ability of the instrument to monitor a flowing solution and generate readings for a solution that is changing composition (for both plutonium concentration and acidity, in this case). It also allows a chance to demonstrate consistency of instrument reading as the conditions lead to the selection of different total plutonium models due to acidity and solution absorbance.

In the experiment, each column was passed through a $1 \mathrm{~mm}$ flow cell $(10 \mathrm{x}$ smaller than the $1 \mathrm{~cm}$ cuvettes described above), so that the entire heart cut would remain below the "saturated" level for the spectrometer. As it happened, the path length was small enough so that the "high absorbance" case was also not accessed.

Figure 6-2 shows the spectrophotometer output for each column elution (solid line) and the analysis results for the observed spectrum for four models: medium and low absorbance for medium and low acidity. The low acidity cases had minimal disproportionation $\left(\mathrm{A}_{830 \mathrm{~nm}}<2.2\right.$ in a 1-mm cell). For the majority of each elution, the measured absorbance and derived acidity were such that clearly only one model would be selected. However, in the middle of each heart cut there are a number of spectra with acidity and/or absorbance near the transition points between categories. For the sake of comparison, spectra for solutions with acidities between 1.2 and 2.8 $\mathrm{M}$ were analyzed with low and medium acidity 
models, and absorbances between 1.9 and 2.5 between 460-510 nm were analyzed with low and medium absorbance models. These ranges bracket any possible misclassification scenarios.

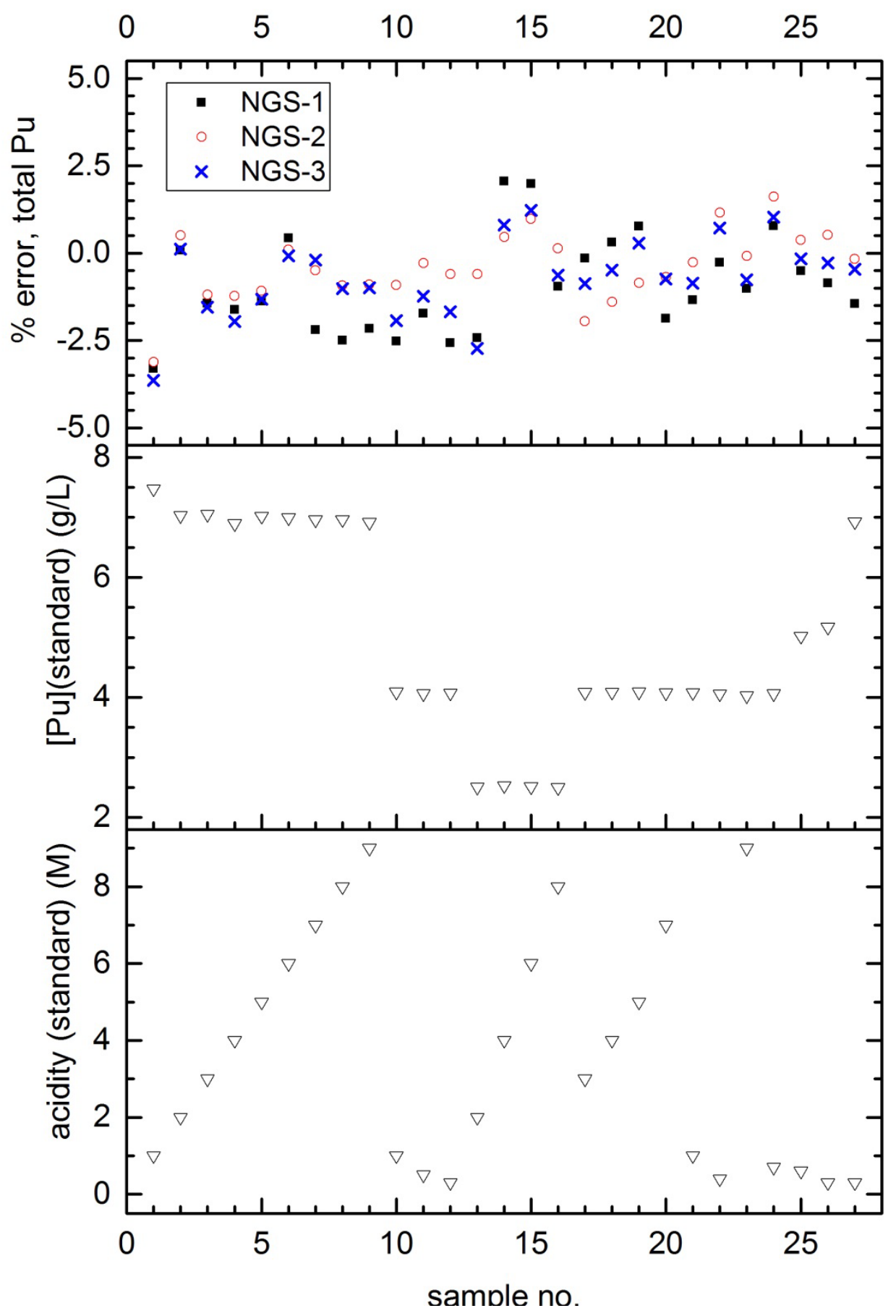

Figure 6-1. Qualification data for Pu standards in cuvettes. 
SRNL-STI-2015-00454

Revision 0

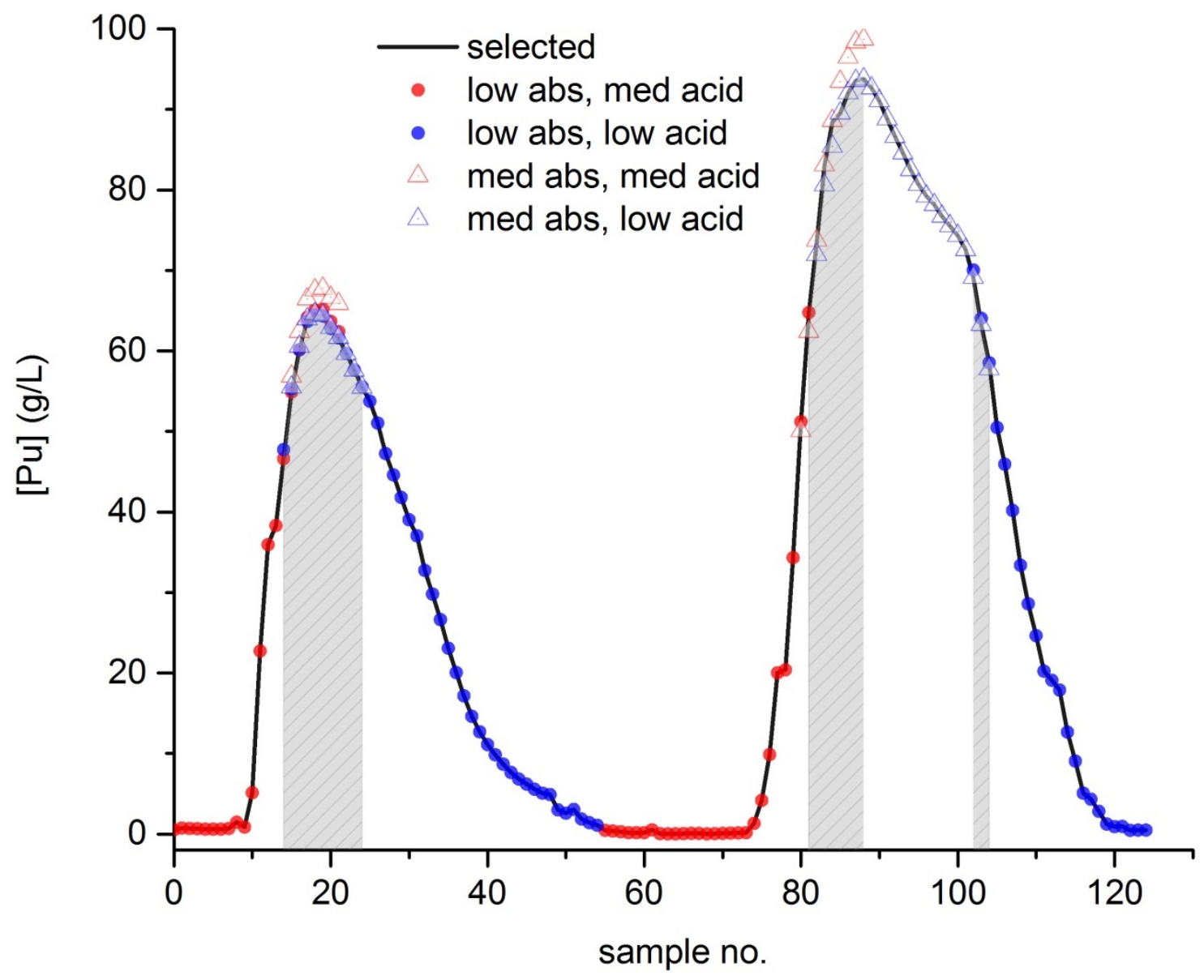

Figure 6-2. Results of monitoring two laboratory Pu column elutions using the spectrophotometer.

For the first elution, the acid/absorbance transitions occurred at samples 14-24. Due to a lower plutonium loading on the column, the peak absorbance did not exceed 2.25. Thus, for samples 15-21, all four models were considered. As can be seen in the figure, the agreement between the models for these spectra is excellent, with an average variance of $1.5 \%$. For the second elution, there are three regions (two contiguous) of potential model overlap. The points where acid and absorbance regions are in transition are decoupled, as there is a higher plutonium loading for this column. For samples 80-81, the absorbance at $460-510 \mathrm{~nm}$ is between $1.9-2.5$, but the acidity is clearly in the "medium" range (> 3M). For samples 82-88, the acidity is decreasing from 2.8 to 1.2, with the absorbance clearly in the "medium" range. As the tail elution starts, at samples 102-104, the absorbance transitions to the "low" range, with the acid clearly "low". The agreement between the possible models in these regions is good, with average variances of $3.0 \%, 4.0 \%$, and $1.3 \%$, respectively. These values are within $2 \sigma$ of the expected uncertainties for the models and thus the differences between the models cannot be considered statistically significant.

Figure 6-3 simulates the spectrophotometer output if these column elutions were conducted in HB-Line, with a 1-inch flow cell. The longer path length would cause the spectrometer to be saturated for most of the elution, as is currently the case for the colorimeters. The transition from high to medium acidity occurs at the earliest part of the head cut, and the medium to low acidity transition occurs before the peak of the elution. Transitions between the absorbance cases are defined consistently with Figure 4-10. 


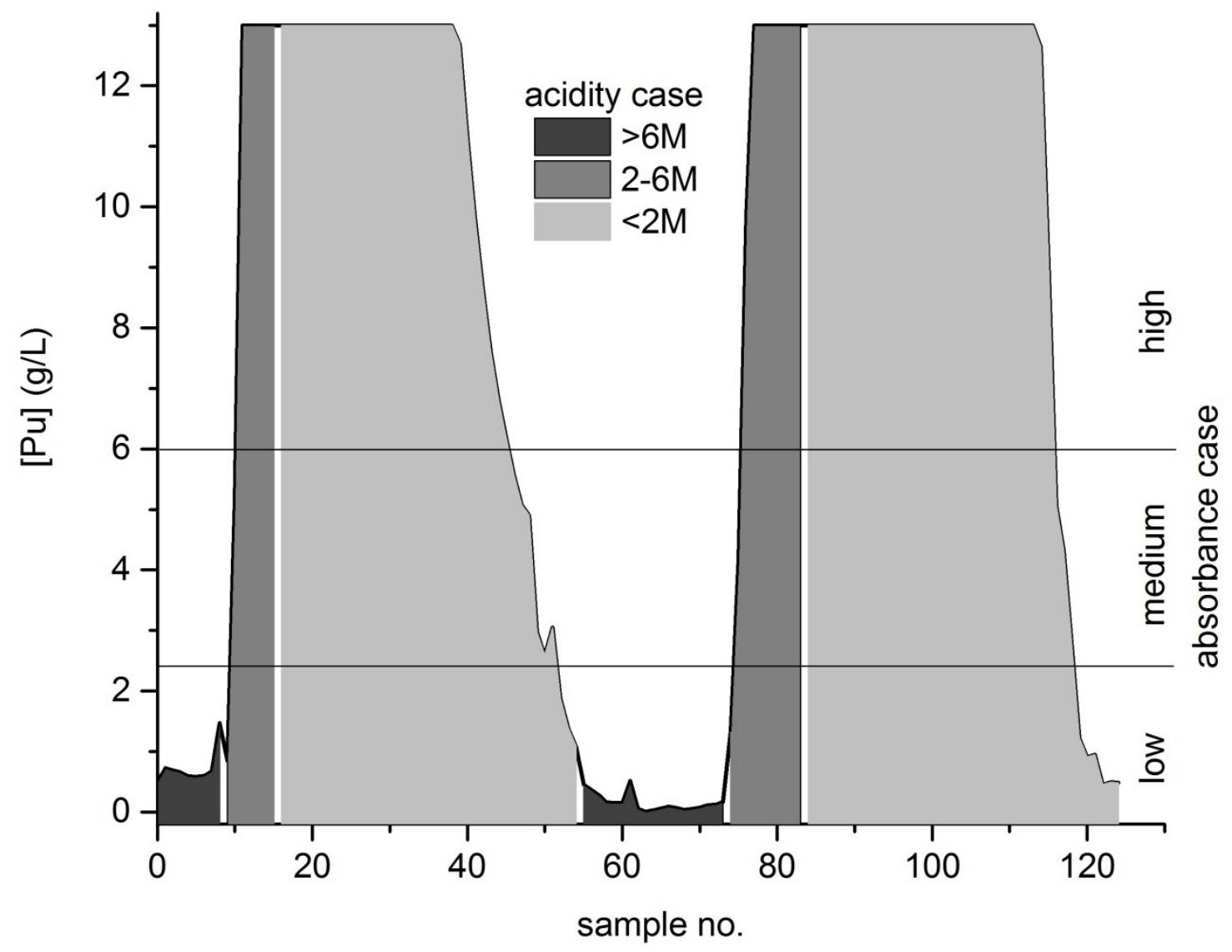

Figure 6-3. Simulated spectrophotometer response for column elutions with 1" flow cell.

Figure 6-3 suggests that the transitions between the low acidity, medium absorbance and low acidity, high absorbance models may be of interest if the tail cut set point is near $6 \mathrm{~g} / \mathrm{L}^{\text {a }}{ }^{\mathrm{a}}$ The difference in output between these two models for spectra observed at that part of the two elutions is $2.8 \%$, which is within the expected uncertainties for the two models.

${ }^{a}$ Some $\mathrm{Pu}^{6+}$ can be detected in these spectra but as the amounts are low ( $<7 \%$ of total $\mathrm{Pu}$ in this particular run) and the overall absorbance is low, only the low-disproportionation models would be invoked. 


\subsection{Conclusions}

SRNL has developed Next Generation Spectrophotometers to replace colorimeters as plutonium process monitors in HB-Line, with specific application to the AFS-2 processing campaign. These instruments address the limitations of the colorimeters that, in the latter, led to high measurement uncertainties associated with nitric acid concentration $(\sim 15 \%(1 \sigma))$ and plutonium oxidation state disproportionation (negative 35\% bias). These uncertainties imposed highly conservative limits in plutonium concentration to preserve nuclear safety. The spectrophotometers are calibrated for solution conditions between 0-13 g/L total plutonium, 0.3-9 M nitric acid, a temperature of 20-50 ${ }^{\circ} \mathrm{C}$, and plutonium disproportionation of up to $20 \%$. The instrument uncertainty depends on the process conditions, but is less than $5 \%(1 \sigma)$ in any of these cases. The spectrophotometers have the capability to measure nitric acid concentration (when plutonium is present in the solution) and the relative amount of the different oxidation states of plutonium $\left(3^{+}, 4^{+}\right.$, and $\left.6^{+}\right)$. They also have internal diagnostics which allow detection of, and in many cases correction for, aspects of instrument health such as lamp aging which can lead to drift of the output if not addressed. These features will lead to steadier output with less maintenance than the colorimeters. The instruments are also compatible with existing optical fibers and flow cells, which simplifies installation.

The development of the NGS has yielded a state of the art analytical instrument with capabilities that are not available with standard, off the shelf diode array spectrophotometers. The most significant improvement is the implementation of real-time correction for both wavelength calibration and intensity response for the two spectrometers in the instrument. The wavelength correction is based on the emission lines from the Xe flash lamp in the instrument, which are continually present in the spectra. The wavelength accuracy improves from $>0.1$ to $0.02 \mathrm{~nm}$. The intensity correction is based on a periodic measurement of stray light, array readout efficiency, and higher order grating effects which expands the linearity of the detector response to over $99.4 \%$ of its total range. The improved performance means that the intensity signals on the two spectrometers (measure and reference) in the spectrophotometer can be accurately ratioed to generate an absorbance spectrum. It also means that spectrometers from different instruments should be much more identical than is normally the case. We have demonstrated that plutonium calibrations can be developed on one spectrophotometer and directly used on many other spectrophotometers with minimal loss of accuracy. Thus, for example, new calibrations can be developed in the laboratory and taken to installed instruments without further modification.

Another important development is the dual light source, combining the outputs of a tungsten-halogen and a Xe flash lamp, and then splitting the light into separate arms for the reference and measure detectors. This arrangement is a feature of the classic double-beam spectrophotometer, but typical diode array systems are based on the single-beam configuration which trades somewhat higher noise levels and sensitivity to lamp fluctuations for convenience and lower cost. The double-beam arrangement essentially provides continual re-referencing of the instrument, so that the absorption spectra and plutonium readings are much less susceptible to drift due to lamp aging (and subsequent color changes). To fully take advantage of the double-beam configuration, custom software has been written to control and read both spectrometers. This allows implementation of other diagnostics that can detect fluctuations in throughput of the flow cell due to turbulence or blockage, loss of signal of one or both lamps, or other parameters.

The spectrophotometers also improve upon the colorimeters in being able to measure the absorbance at many wavelengths simultaneously, rather than being restricted to two narrow wavelength regions. The wavelength range sampled by the spectrophotometer is sufficiently broad that specific changes due to acidity, temperature, oxidation state, background interferences, and other causes can be observed and distinguished. Plutonium calibration models take advantage of this additional information to find combinations of the absorbance that generate plutonium readings which are nominally independent of these confounding factors. The complexity of plutonium chemistry over these conditions is sufficient to 
compromise the accuracy of a single, global prediction model. By classifying spectra as being within a subset of the overall condition range, one can instead apply a localized prediction model which is less complex and more accurate and robust. A potential disadvantage of this approach is that multiple prediction models need to be made, but in practice this activity does not involve much more effort than would already be expended towards a single, global calibration.

\subsection{Future opportunities}

The spectrophotometers are generalizable to other process analysis situations for which absorption spectroscopy would be suited. For example, there are numerous locations in H Canyon (dissolvers and First and Second Uranium Cycle) where spectrophotometers and colorimeters have been used or tested for analysis of uranium and plutonium. Previous HB-Line campaigns have purified neptunium, using appropriately configured colorimeters to monitor anion exchange column effluent. Developing new NGS instruments of this design for those applications would be comparatively straightforward and provide improved performance over the previous instruments.

There are also potential improvements for the current application to HB-Line which were outside the scope of the project but which would provide a benefit to the facility if they were implemented in the future. The NK-1 and NK-3 flow cells have a 1" path length, and the maximum plutonium concentration which can be measured is $13 \mathrm{~g} / \mathrm{L}$. As is the case for the colorimeters, the spectrophotometer signal will saturate for most of the elution (see Figure 6-3), making it impossible to estimate the amount of plutonium in the different cuts from the elution, the real-time acidity of the solution, any changes in the elution profile which might indicate resin degradation, or other processing parameters. These capabilities can be added by introducing a flow cell with a smaller path length. For example, a 0.1" path length would increase both ends of the detection range by 10x (e.g. $0.2-130 \mathrm{~g} / \mathrm{L}$ ). Sensitivity to current setpoints would be maintained with this configuration. Development work would confirm that flow characteristics (both in terms of turbulence inside the cell and of the elution profile) could be maintained with the smaller cell. Spectrophotometer output would be corrected by the multiplicative factor, which is part of a configuration file.

Another improvement would come from installing a run of optical fiber from the spectrophotometers to the Lab Line in the HB-Line. This would permit use of the glove boxes, fibers, and cuvette holders in that laboratory to measure plutonium standards directly. These measurements would be an additional check on the performance of the spectrophotometers. 


\subsection{References}

[1] R.J. Lascola, "Use of Pu Colorimeter for Tail Cut Monitoring in AFS-2 Processing”, SRNL-STI2012-00565, SRNL, Aiken, SC (September 2012).

[2] R.J. Lascola and E.A. Kyser, "Experimental Determination of Colorimeter Bias for Pu Measurements in AFS-2 Processing”, SRNL-STI-2013-00655, SRNL, Aiken, SC (March 2013).

[3] C.F. Metz, “The Analytical Chemistry of Plutonium”, Anal. Chem., 29, 1748-1756 (1957).

[4] D.K. Viers et al., "Characterization of the Nitrate Complexes of Pu(IV) Using Absorption Spectroscopy, ${ }^{15} \mathrm{~N}$ NMR, and EXAFS”, J. Alloys Compounds, 213/214, 328-332 (1994).

[5] W.L. Kubik, Jr. and J.C. Jackson, “A Thermodynamic Model of Plutonium (IV) Nitrate Solutions”, J. Radioanal. Nucl. Chem., 293, 601-612 (2012).

[6] E.A. Kyser and P.E. O’Rourke, “Extent of Disproportionation During the HB-Line Anion Exchange Column Elution Cycle”, SRNL-L3100-2013-00184, SRNL, Aiken, SC (October 2013).

[7] R.J. Lascola, "Characterization of $\mathrm{H}$ Canyon Colorimeter Performance at Extended Uranium Concentrations” WSRC-STI-2007-00217, SRNL, Aiken, SC (April 2007).

[8] A. Kramida, Yu. Ralchenko, and J. Reader, NIST Atomic Spectra Database (ver. 5.2). [Online] National Institute of Standards and Technology, 2014. [Cited: July 28, 2015.] http://physics.nist.gov/asd.

[9] H.A. Strobel and W.R. Heineman, Chemical Instrumentation: A Systematic Approach, Third Edition. New York : John Wiley \& Sons, 1989.

[10] P. Fleming, "Stray-Light", [ed.] C. Burgess and T. Frost. Standards and Best Practice in Absorption Spectrometry. Oxford : Blackwell Science, Ltd., 1999, Chapter 6.

[11] Y.T. Kim and I. An, "Order-sorting filters for a grating spectrometer and multichannel detection system: application to real-time spectroscopic ellipsometry”, Anal. Chem., 70, 1346-1351 (1998).

[12] R. Mavrodineanu and J.R. Baldwin, "Metal-On-Quartz Filters as a Standard Reference Material for Spectrophotometry - SRM 2031.” SP 260-68, National Institute of Standards and Technology. Washington, DC (April 1980).

[13] D.W. Allen, "Holmium Oxide Glass Wavelength Standards”, J. Res. Natl. Inst. Stand. Technol., 112, 303-306 (2007).

[14] R.J. Lascola, “On Line Spectrophotometric Measurement of Uranium and Nitrate in H Canyon”, WSRC-TR-2002-00334, Savannah River Technology Center. Aiken, SC (July 2002).

[15] F. Weigel, J.J. Katz, and G.T. Seaborg, "Plutonium”, [ed.] J. J. Katz, G. T. Seaborg and L. T. Morss. The Chemistry of the Actinide Elements. 2nd Edition. New York : Chapman and Hall, 1986, Vol. 1, Chapter 7. 
[16] P. Geladi and B.R. Kowalski, "Partial Least-Squares Regression: A Tutorial”, Anal. Chim. Acta, 185, 1-17 (1986).

[17] K.R. Beebe, R.J. Pell, and M.B. Seasholtz, Chemometrics: A Practical Guide. New York : Wiley, 1998.

[18] C.E. Miller, “Chemometrics in Process Analytical Chemistry”, [ed.] K. A. Bakeev. Process Analytical Technology. Ames, Iowa : Blackwell Publishing, 2005, Chapter 8.

[19] J. Workman, Jr. et al., "Process Analytical Chemistry”, Anal. Chem., 83, 4557-4578 (2011).

[20] R.J. Lascola, et al., "Actinide Analysis by Absorption Spectroscopy - Method Development and Performance”, WSRC-STI-2007-00465, SRNL, Aiken, SC (August 2007).

[21] J.M. Berg et al., "Speciation Model Selection by Monte Carlo Analysis of Optical Absorption Spectra: Plutonium(IV) Nitrate Complexes”, Appl. Spectrosc. 54, 812-823 (2000).

[22] A. Savitzky and M.J.E. Golay, "Smoothing and Differentiation of Data by Simplified LeastSquares Procedures”, Anal. Chem., 36, 1627-39 (1964).

[23] P.I. Artyukihn, V.I. Medvedovskii, and A.D. Gel'man, "Disproportionation of Pu(IV) and Pu(V) in Nitric Acid Solutions, Russ. J. Inorg. Chem., 4, 596-599 (1959).

[24] J.M. Haschke, "Disproportionation of Pu(IV): A Reassessment of Kinetic and Equilibrium Properties”, J. Nucl. Materials, 362, 60-74 (2007).

[25] D.E. Fauerby, “Instrumentation Uncertainties Evaluation - Pu Spectrophotometer Indication for Raffinate Line and Columns NK-1 and NK-3”, J-CLC-H-01078, Rev. 0. Savannah River Site (2015).

[26] R.J. Lascola, “Colorimeter Development for Np Measurements in HB-Line”, WSRC-TR-200300525, Savannah River Technology Center, Aiken, SC (November 2003).

[27] K.P. Crapse et al., "AFS-2 Flowsheet Modifications to Address the Ingrowth of Pu(VI) During Metal Dissolution”, SRNL-STI-2013-00709, SRNL, Aiken, SC (July 2014). 


\section{Distribution:}

M.J. Barnes, 773-A

T. M. Bolen, 221-H

T. B. Brown, 773-A

K.P. Burrows, 704-2H

C.M. Gregory, 773-A

I.T. Clark, 704-2H

D.M. Immel, 781-A

B.J. Collins, 704-2H

E.A. Kyser, 773-A

S. Hudlow, 221-H

R.J. Lascola, 999-2W

W.R. Jeffords, 704-2H

S. L. Marra, 773-A

C.E. Johnson, 704-2H

A.M. Murray, 773-A

C.D. Loyal, 704-2H

T.A. Nance, 781-A

J. Mancilla, 704-2H

P.E. O’Rourke, 773-A

A.T. Masterson, 704-2H

J.R. Plummer, 781-A

T.D. Nguyen, 705-H

L.E. Roy, 703-41A

K. D. Scaggs, 704-2H

E.T. Sadowski, 773-A

J. B. Schaade, 704-2H

L.T. Sexton, 773-42A

L. Scott, Jr., 704-2H

T.F. Severynse, 707-24B

B.L. Sims, Jr., 221-H

Records Administration (EDWS)

P.W. Singletary, 221-17H

D. Stimac, 704-2H

J. E. Therrell, 704-2H

T.M. Tran, 707-C

T. E. Worth, 704-2H 